\title{
TRACE AS AN ALTERNATIVE DECATEGORIFICATION FUNCTOR
}

\author{
ANNA BELIAKOVA, ZAUR GULIYEV, KAZUO HABIRO, AND AARON D. LAUDA
}

\begin{abstract}
Categorification is a process of lifting structures to a higher categorical level. The original structure can then be recovered by means of the so-called "decategorification" functor. Algebras are typically categorified to additive categories with additional structure and decategorification is usually given by the (split) Grothendieck group. In this article we study an alternative decategorification functor given by the trace or the zeroth Hochschild-Mitchell homology. We show that this form of decategorification endows any 2-representation of the categorified quantum $\mathfrak{s l}_{n}$ with an action of the current algebra $\mathbf{U}\left(\mathfrak{s l}_{n}[t]\right)$ on its center.
\end{abstract}

\section{INTRODUCTION}

1.1. What is categorification? Categorification is perhaps best explained by defining decategorification. Quite broadly, decategorification is a rigorously defined procedure for forgetting information and reducing the complexity of a given mathematical structure. A bit more formally, a decategorification can be thought of as a map

$$
(n-1) \text {-category } \longleftarrow \mathcal{D} \text {-category }
$$

for simplifying an $n$-categorical structure into an $(n-1)$-categorical structure.

Categorifcation asks the question "given a specific $(n-1)$-category $A$, find an $n$-category $B$ such that $A \cong \mathcal{D}(B)$ ". In this case, we say that $A$ is the decategorification of $B$ and that $B$ categorifies $A$. Often it is the case that the existence of a categorification of $A$ reveals new insights and hidden structure that could not be seen without knowledge of $B$. For more introductory material on categorification see [2, 35, 38, 58].

An example when $n=1$ can be obtained by considering the 1-category $\mathbf{V e c t}_{\mathbb{k}}$ of finite dimensional $\mathbb{k}$-vector spaces. We can define a decategorification

$$
\mathbb{N} \longleftarrow \text { Vect }_{\mathrm{k}}
$$

from the 1-category Vect $_{k}$ to the 0-category, or set, of natural numbers $\mathbb{N}$ by setting $\mathcal{D}=\operatorname{dim}$. In this example a natural number $n$ is categorified by any vector space $V$ with $\operatorname{dim}(V)=n$. This categorification lifts much of the structure of natural numbers, i.e. $\operatorname{dim}(V \oplus W)=\operatorname{dim}(V)+\operatorname{dim}(W)$ and $\operatorname{dim}(V \otimes W)=\operatorname{dim}(V) \times \operatorname{dim}(W)$.

Generalizing the previous example, let $\mathbf{g V e c t}_{\mathbb{k}}$ denote the category of graded vector spaces $V=$ $\oplus_{k \in \mathbb{Z}} V_{k}$. One way to decategorify a graded vector space is to take its graded dimension

$$
\mathbb{N}[q, q]^{-1} \longleftarrow{\mathcal{D}=\operatorname{dim}_{q}}_{\mathbf{g V e c t}_{\mathbb{k}}}
$$

where

$$
\operatorname{dim}_{q} V:=\sum_{k \in \mathbb{Z}} q^{k} \operatorname{dim} V_{k}
$$

Date: September 4, 2014. 
This example can be extended another direction by considering the category Kom( $\left.\mathbf{g V e c t}_{\mathrm{k}}\right)$ of complexes of graded vector spaces. The graded Euler characteristic gives rise to a decategorification map taking a bounded complex of graded vector spaces into a Laurent polynomial $\chi(C \bullet)=\sum_{i}(-1)^{i} \operatorname{dim}_{q} C^{i} \in$ $\mathbb{Z}\left[q, q^{-1}\right]$. While these examples may seem somewhat trivial, these elementary ideas resurface in more sophisticated categorifications discussed below.

Several important observations are in order. A categorification need not be unique, in the sense that there may be two different $n$-categories $B$ and $B^{\prime}$ with $A=\mathcal{D}(B)=\mathcal{D}\left(B^{\prime}\right)$. Furthermore, there may be several different ways to simplify a given $n$-categorical structure into an $(n-1)$-categorical structure. That is to say, it may be possible to decategorify a given structure in more than one way. In this article we will focus on two ways of decategorifying an (additive) category to produce an abelian group. These are the "Grothendieck group" and "trace".

1.2. The Grothendieck group. For any additive category $\mathcal{C}$, its split Grothendieck group $K_{0}(\mathcal{C})$ is the abelian group generated by isomorphisms classes of its objects $\{[X] \cong \mid X \in \mathrm{Ob}(\mathcal{C})\}$, modulo the relation that $[A \oplus B]_{\cong}=[A]_{\cong}+[B]_{\cong}$. Categories are organized into a 2-categorical structure Cat consisting of categories, functors, and natural transformations. (Here and throughout this article we ignore issues of size of categories.) Put into the framework described above, the (split) Grothendieck group can be thought of as the procedure for turning the 2-categorical structure of an (additive) category into a 1-categorical structure of an abelian group.

$$
\mathrm{Ab} \longleftarrow \mathcal{D}=K_{0} \quad \text { AdCat. }
$$

We showed above that we can decategorify a vector space $V$ into a natural number by taking its $\operatorname{dimension} \operatorname{dim}(V)$ as a decategorification map. This map shows up again when we decategorify the category of vector spaces Vect $_{\mathrm{k}}$ using the Grothendieck group decategorification map. By choosing a basis, every vector space is isomorphic to a direct sum of copies of the ground field $[V] \cong=\left[\mathbb{k}^{\operatorname{dim}(V)}\right] \cong=$ $\operatorname{dim}(V)[\mathrm{k}] \cong$ for any $V \in \mathrm{Ob}\left(\mathbf{V e c t}_{\mathrm{k}}\right)$. Hence, the Grothendieck group $K_{0}\left(\mathbf{V e c t}_{\mathrm{k}}\right)$ of the category of $\mathbb{k}$-vector spaces can be identified with the infinite cyclic abelian group $\mathbb{Z}$ after sending $[\mathbb{k}]_{\cong \mapsto 1}$.

If $\mathcal{C}$ is a graded additive category, then the Grothendieck group $K_{0}(\mathcal{C})$ acquires the structure of a $\mathbb{Z}\left[q, q^{-1}\right]$-module by declaring that $[x\langle t\rangle] \cong=q^{t}[x] \cong$. For example, the split Grothendieck group of the graded additive category of graded vector spaces gVect $_{\mathrm{k}}$ can be identified with $\mathbb{Z}\left[q, q^{-1}\right]$ by sending $[\mathbb{k}] \cong \rightarrow 1$ since

$$
[V] \cong=\left[\bigoplus_{k \in \mathbb{Z}} V_{k}\right] \cong=\sum_{k \in \mathbb{Z}} q^{k}\left[V_{k}\right] \cong=\sum_{k \in \mathbb{Z}} q^{k} \operatorname{dim} V_{k}[\mathbb{k}] \cong=\operatorname{dim}_{q} V[\mathbb{k}] \cong .
$$

Again, we see our previous decategorification map $\operatorname{dim}_{q}$ sending a graded vector spaces to $\mathbb{N}\left[q, q^{-1}\right]$ appearing in the context of the split Grothendieck group taking the category of graded vector spaces gVect $_{\mathrm{k}}$ into the abelian group $K_{0}\left(\right.$ gVect $\left._{\mathrm{k}}\right)$.

1.2.1. The Grothendieck group and Euler characteristics. The Grothendieck group has a universal property making it the universal way of producing an abelian group from an additive category. This universal property makes the Grothendieck group into a universal receptacle for generalized Euler characteristics.

If we denote by $\operatorname{Kom}\left(\mathbf{g V e c t}_{\mathbb{k}}\right)$ the category of bounded complexes of graded vector spaces, then the graded Euler characteristic gives rise to a decategorification map

$$
K_{0}\left(\text { gVect }_{\mathrm{k}}\right) \longleftarrow x
$$

given by taking a complex $C^{\bullet}$ of graded vector spaces into a Laurent polynomial

$$
\chi\left(C^{\bullet}\right)=\sum_{i}(-1)^{i}\left[C^{i}\right] \cong=\sum_{i}(-1)^{i} \operatorname{dim}_{q}\left(C^{i}\right)[\mathbb{k}] \in K_{0}\left(\mathbf{g V e c t}_{\mathrm{k}}\right) \cong \mathbb{Z}\left[q, q^{-1}\right] .
$$


More generally, for any additive category $\mathcal{C}$, the Euler characteristic $\chi\left(C^{\bullet}\right)=\sum_{i}(-1)^{i}\left[C^{i}\right] \cong$ of a complex in $\operatorname{Kom}(\mathcal{C})$ is element of the Grothendieck group $K_{0}(\mathcal{C})$. Thus, we see that the Euler characteristic fits nicely with the Grothendieck group decategorification map.

1.3. The trace decategorification map. The trace, or zeroth Hochschild homology, is another procedure for turning a linear category $\mathcal{C}$ into an abelian group. It is defined by

$$
\operatorname{Tr}(\mathcal{C}):=\bigoplus_{x \in \operatorname{Ob}(\mathcal{C})} \mathcal{C}(x, x) / \operatorname{Span}\{f g-g f\}
$$

where $f$ and $g$ run through all pairs of morphisms $f: x \rightarrow y$ and $g: y \rightarrow x$ with $x, y \in \mathrm{Ob}(\mathcal{C})$. We write $[f]$ for the class of an endomorphism $f: x \rightarrow x$ in $\operatorname{Tr}(\mathcal{C})$. In section 3 we show that the trace satisfies the property that $[f \oplus g]=[f]+[g]$.

In the category Vect $_{\mathrm{k}}$, the class $[\phi]$ of an endomorphism $\phi: V \rightarrow V$ is equal to the class $\operatorname{tr}(\phi)\left[1_{\mathrm{k}}\right]$, where $\operatorname{tr}(\phi)$ is the usual trace of the linear endomorphism $\phi$ (see exercise 2). Hence, the categorical trace of Vect ${ }_{k}$ is closely connected to the usual trace map sending a vector space to an element of the ground field $\mathbb{k}$.

Note that $\operatorname{dim}_{V}=\operatorname{tr}\left(1_{V}\right)$ so that the trace can be seen as a generalization of the dimension decategorification map. In Section 3 we show that the trace $\operatorname{Tr}\left(\mathbf{V e c t}_{\mathbb{k}}\right)$ of the category of vector spaces can be identified with the ground field $\mathbb{k}$ and that the trace $\operatorname{Tr}\left(\mathbf{g V e c t}_{\mathbb{k}}\right)$ of the category of graded vector spaces is isomorphic to $\mathbb{k}\left[q, q^{-1}\right]$.

1.3.1. Traced Euler characteristics. The Euler characteristic in Vect $_{k \mathrm{k}}$ can be recast in the language of traces by observing that $\operatorname{dim}_{V}=\operatorname{tr}\left(1_{V}\right)$, which allows to define so called traced Euler characteristic

$$
\chi_{\operatorname{tr}}\left(C^{\bullet}\right)=\sum_{i}(-1)^{i} \operatorname{tr}\left(1_{C^{i}}\right) .
$$

This notion can be extended to any linear category $\mathcal{C}$ as follows. Given a complex $C^{\bullet} \in \operatorname{Kom}(\mathcal{C})$,

$$
\operatorname{Tr}(\mathcal{C}) \longleftarrow \chi_{t r} \operatorname{Kom}(\mathcal{C})
$$

is defined by setting $\chi_{t r}\left(C^{\bullet}\right)=\sum_{i}(-1)^{i}\left[1_{C^{i}}\right]$, where $[f]$ denotes the class of the endomorphism $f$ in $\operatorname{Tr}(\mathcal{C})$. In general, $\chi_{\operatorname{tr}}$ does not need to coincide with the usual Euler characteristic.

1.4. Comparing Grothendieck group and trace. We have now described two flavors of decategorification. One which we will refer to as the Grothendieck decategorification which is closely connected to the notion of dimension, graded dimension, and Euler characteristic. Our second notion of decategorification, which we will call the trace decategorification, seems to generalize the Grothendieck group decategorification in much the same way that the trace of linear map generalizes the dimension of a vector space $\operatorname{dim}(V)=\operatorname{tr}\left(1_{V}\right)$. The trace decategorification is closely connected with the usual trace and it gives rise to a notion of traced Euler characteristic.

These two notions of decategorification can actually produce the same results in some very interesting examples. One of the most spectacular examples is the categorification of the Jones polynomial by the Khovanov complex of graded $\mathbb{Z}$-modules, where the Jones polynomial can be recovered as the graded Euler characteristic 31, 32. Bar-Natan generalized Khovanov's construction by defining the so-called Khovanov bracket: a complex over an additive category of special 2-dimensional cobordisms. In his setting, one recovers the Jones polynomial by means of the traced Euler characteristic (Theorem 6 [4]). Hence, the functor Tr or the zeroth Hochschild-Mitchell homology replaces $K_{0}$ in the Bar-Natan setting.

This survey paper is devoted to the comparison of $K_{0}$ and $\mathrm{Tr}$ as decategorification functors. The interplay between these two notions of decategorification is surprisingly rich. It can be thought of as a categorical counterpart to the Chern character map, see for example [16]. 
1.5. Quantum group categorifications. There is a strong interplay between quantum link invariants and algebraic objects known as quantum groups. Given the existence of link homology theories categorifying link invariants, it is perhaps not surprising to discover that quantum groups can also be categorified. In this article we will examine how the two notions of decategorification given by the Grothendieck group and the trace can be applied to categorified quantum groups.

Quantum groups are Hopf algebras associated to Lie algebras $\mathfrak{g}$. They are obtained by $q$-deforming the universal enveloping algebra $\mathbf{U}(\mathfrak{g})$. Lusztig's modified, or idempotented, form $\dot{\mathbf{U}}(\mathfrak{g})$ of the quantum group $\mathbf{U}_{q}(\mathfrak{g})$ is well suited for categorification. These algebras are equipped with a family of orthogonal idempotents $1_{\lambda}$ indexed by the weight lattice $X$ of $\mathfrak{g}$. Any $\mathbb{k}$-algebra $A$ equipped with orthogonal idempotents indexed by a set $X$ can be regarded as an additive category whose objects are the elements of $X$. Given two elements $\lambda, \mu \in X, \mathbb{k}$-vector space of maps from $\lambda$ to $\mu$ can be defined by $1_{\mu} A 1_{\lambda}$. In this framework, the idempotents $1_{\lambda}$ are thought of as identity morphisms from the object $\lambda$ to itself. Composition in the category

$$
1_{\mu^{\prime}} A 1_{\lambda^{\prime}} \times 1_{\mu} A 1_{\lambda} \rightarrow \delta_{\mu, \lambda^{\prime}} 1_{\mu^{\prime}} A 1_{\lambda}
$$

is given by multiplication in $A$.

By thinking of a quantum group $\mathbf{U}_{q}(\mathfrak{g})$ as a linear category in this way, we are naturally led to consider a categorification of $\mathbf{U}_{q}(\mathfrak{g})$ given by the structure of a 2-category and our decategorification map must reduce a linear 2-category to a linear 1-category.

Associated to a field $\mathbb{k}$ and a symmetrizable Kac-Moody algebra $\mathfrak{g}$, a 2-category $\dot{\mathcal{U}}(\mathfrak{g})$ was defined in 33 generalizing the $\mathfrak{s l}_{2}$ case from [37. The 2-category $\dot{\mathcal{U}}(\mathfrak{g})$ is the idempotent completion, or Karoubi envelope, of a 2-category $\mathcal{U}(\mathfrak{g})$ defined in terms of a graphical calculus. More precisely, the objects of the category $\mathcal{U}(\mathfrak{g})$ are indexed by the weight lattice $X$ of $\mathfrak{g}$. The morphisms are formal direct sums of composites of maps $\mathbf{1}_{\lambda}: \lambda \rightarrow \lambda, \mathcal{E}_{i} \mathbf{1}_{\lambda}: \lambda \rightarrow \lambda+\alpha_{i}$, and $\mathcal{F}_{i} \mathbf{1}_{\lambda}: \lambda \rightarrow \lambda-\alpha_{i}$ corresponding to the Chevalley basis of $\mathfrak{g}$. For each 1-morphism $x$ there is also a grading shifted 1-morphism $x\langle t\rangle$ in $\mathcal{U}(\mathfrak{g})$. The 2morphisms are given by $\mathbb{k}$-linear degree preserving combinations of certain planar diagrams modulo local relations. See Figure 1 for an example of planar diagram in $\mathcal{U}(\mathfrak{g})$.

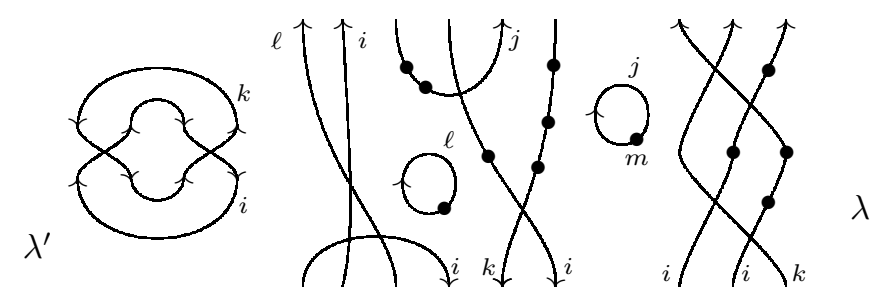

FiguRE 1. An example of a diagram representing a 2-morphism in $\mathcal{U}(\mathfrak{g})$ from $\mathcal{E}_{i}^{2} \mathcal{E}_{\ell} \mathcal{F}_{i} \mathcal{F}_{k} \mathcal{F}_{i} \mathcal{E}_{i} \mathcal{E}_{i} \mathcal{E}_{k} \mathbf{1}_{\lambda}$ to $\mathcal{E}_{\ell} \mathcal{E}_{i} \mathcal{F}_{j} \mathcal{F}_{j} \mathcal{F}_{i} \mathcal{E}_{j} \mathcal{E}_{i} \mathcal{E}_{k} \mathcal{E}_{i} \mathbf{1}_{\lambda}\langle t\rangle$. The degree $\langle t\rangle$ of this 2-morphism is determined by the relationship between the simple roots $i, j, k, \ell$ in the Cartan datam associated to $\mathfrak{g}$.

Extending the split Grothendieck group decategorification map for linear categories, it is possible to define the split Grothendieck group $K_{0}$ of a linear 2-category. It was shown in 33 for $\mathfrak{g}=\mathfrak{s l}_{n}$ that applying this notion of decategorification to the Karoubi envelope $\dot{\mathcal{U}}\left(\mathfrak{s l}_{n}\right)$ of the 2-category $\mathcal{U}\left(\mathfrak{s l} l_{n}\right)$ produces the integral idempotented form of the quantum enveloping algebra $\mathbf{U}_{q}\left(\mathfrak{s l}_{n}\right)$. Hence, with this notion of decategorification the 2-category $\dot{\mathcal{U}}\left(\mathfrak{s l}_{n}\right)$ can be viewed as a categorification of the quantum group $\mathbf{U}_{q}\left(\mathfrak{s l}_{n}\right)$. The $n=2$ case of this result was proven in [37] and the case of general $\mathfrak{g}$ appears in 64. For a closely related construction see [57. 
More recently it has been shown that using a 2-categorical analog of the trace as the decategorification map produces some very rich structures [8, 65, 7]. The trace relation can be realized diagrammatically by considering the diagrams for $\mathcal{U}(\mathfrak{g})$ on an annulus.

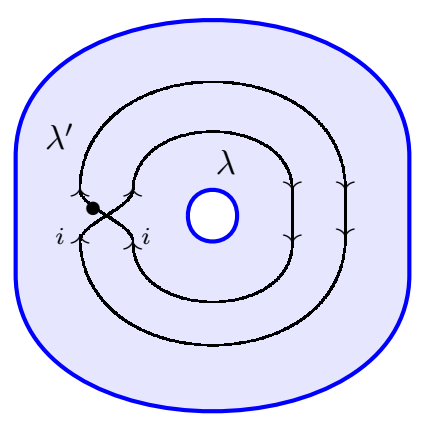

The invariance of the graphical calculus of $\mathcal{U}(\mathfrak{g})$ under planar deformations allows us to slide portions of the diagram around the annulus imposing the trace relation.

1.6. Traces of categorified quantum groups. In this article we hope to emphasize that the trace decategorification map $T r$ has various advantages to $K_{0}$.

- Using $\operatorname{Tr}$ we can work with the 2-category $\mathcal{U}(\mathfrak{g})$ rather than its Karoubi envelope, since $\operatorname{Tr}$ does not change under the passage to the Karoubi envelope. This is a nice advantage as the passage to the Karoubi envelope often takes us out of the purely diagrammatic world.

- Trace is defined for linear categories; in order for $K_{0}$ to be defined the category needs to be additive.

- The trace can have a richer structure than $K_{0}$ as is demonstrated by Theorems 5.1 and 6.2 .

We outline the methods and tools used in studying $K_{0}$ and traces for categorified quantum groups.

1.6.1. Trace and $K_{0}$ of $\dot{\mathcal{U}}$. Just as the Euler characteristic and traced Euler characteristic agree for Khovanov homology, we will explain that the 2 -categories $\mathcal{U}(\mathfrak{g})$ simultaneously categorify the quantum group $\mathbf{U}\left(\mathfrak{s l}_{n}\right)$ via the Grothendieck group decategorification functor $K_{0}$ and the trace decategorification functor Tr.

Theorem 1.1 ([33, 7]). Let $\mathbb{k}$ be a field of characteristic 0 and $\mathfrak{g}=\mathfrak{s l}_{n}$. Then there are isomorphisms

$$
\operatorname{Tr}(\mathcal{U}) \cong K_{0}(\dot{\mathcal{U}}) \otimes_{\mathbb{Z}} \mathbb{k} \cong \dot{\mathbf{U}}
$$

and $\mathrm{HH}_{i}(\mathcal{U})=0$ for $i>0$.

Again, this result is somewhat surprising since these two decategorification procedures need not agree in general. We will explain that there is a large class of examples closely connected to geometric settings where this phenomenon is likely to occur.

Very recently a direct connection was established between categorified quantum groups and $\mathfrak{s l}_{n}$ link homology theories [40, 54. Working in an enhanced foam category introduced by Christian Blanchet, one can realize all the foam relations in a Bar-Natan like setting as arising from the relations in the categorified quantum group $\mathcal{U}\left(\mathfrak{s l}_{n}\right)$ via so called foamation functors first studied in [48. Once translated through these foamation functors, the theorem above immediately implies that the traced and usual Euler characteristics in the $\mathfrak{s l}_{n}$ link homology theories coincide.

Corollary 1.2. In $\mathfrak{s l}_{n}$ link homology theories, traced and usual Euler characteristics do coincide. 
1.6.2. Trace and $K_{0}$ of $\mathcal{U}^{*}$. By allowing homogeneous, but not necessarily grading preserving 2morphisms in $\mathcal{U}(\mathfrak{g})$ we can define a version of the 2-category with larger 2-hom spaces. Let $\mathcal{U}^{*}(\mathfrak{g})$ denote the 2-category with the same objects and one morphisms as $\mathcal{U}(\mathfrak{g})$, but with 2-hom spaces between one morphisms $X \mathbf{1}_{\lambda}$ and $Y \mathbf{1}_{\lambda}$ given by

$$
\mathcal{U}^{*}(\mathfrak{g})\left(X \mathbf{1}_{\lambda}, Y \mathbf{1}_{\lambda}\right):=\bigoplus_{t \in \mathbb{Z}} \mathcal{U}(\mathfrak{g})\left(X \mathbf{1}_{\lambda}, Y \mathbf{1}_{\lambda}\langle t\rangle\right) .
$$

One can alternatively think of $\mathcal{U}^{*}$ as the result of adding isomorphisms $X \mathbf{1}_{\lambda} \rightarrow X \mathbf{1}_{\lambda}\langle t\rangle$ for all $t \in \mathbb{Z}$. This essentially kills the grading making Grothendieck ring $K_{0}\left(\mathcal{U}^{*}\right)$ only a $\mathbb{Z}$-module, rather than a $\mathbb{Z}\left[q, q^{-1}\right]$-module. While this version of the 2 -category is less interesting from a $K_{0}$ perspective, it has interesting consequences for the trace.

The trace of the graded version $\mathcal{U}^{*}$ of the categorified quantum $\mathfrak{s l}_{2}$ is isomorphic to the idempotent form of the current algebra $\mathbf{U}\left(\mathfrak{s l}_{2}[t]\right):=\mathbf{U}\left(\mathfrak{s l}_{2} \otimes \mathbb{Q}[t]\right)[\underline{8}$. In $[8$ it is also shown that for the integral version $\mathcal{U}_{\mathbb{Z}}\left(\mathfrak{s l}_{2}\right)(\mathbb{k}$ is replaced by $\mathbb{Z}$ in the definition), we have

$$
\operatorname{Tr}\left(\mathcal{U}_{\mathbb{Z}}\right)=K_{0}\left(\dot{\mathcal{U}}_{\mathbb{Z}}\right)=K_{0}(\dot{\mathcal{U}}) .
$$

It is interesting that one loses the $q$ by considering the graded category $\mathcal{U}^{*}$, but one sees the positive part of the loop algebra $\mathfrak{s l}_{2} \otimes \mathbb{Q}\left[t, t^{-1}\right]$ appear.

We expect that these results generalize to finite type simply-laced Kac-Moody algebras $\mathfrak{g}$. However, to ease the exposition in this article, we set $\mathfrak{g}=\mathfrak{s l}_{n}$ and describe evidence for the following conjecture in section 5.2

Conjecture 1. For $\mathfrak{g}=\mathfrak{s l}_{n}$, we have

$$
K_{0}\left(\dot{\mathcal{U}}_{\mathbb{Z}}\right)=\operatorname{Tr}\left(\mathcal{U}_{\mathbb{Z}}\right)=K_{0}(\dot{\mathcal{U}})
$$

and $\operatorname{Tr}\left(\mathcal{U}_{\mathbb{Z}}^{*}\right)$ coincides with the integral idempotented version of the current algebra $\mathbf{U}\left(\mathfrak{s}_{n}[t]\right)$ defined in Section 5.2 ,

In this paper we construct a homomorphism $\mathbf{U}\left(\mathfrak{s l}_{n}[t]\right) \rightarrow \operatorname{Tr}\left(\mathcal{U}^{*}\left(\mathfrak{s l}_{n}\right)\right)$ utilizing the graphical calculus and verifying relations directly (see Proposition 23). This implies that the center of objects $Z(\dot{\mathcal{U}}):=$ $\bigoplus_{\lambda \in X} \operatorname{End}_{\dot{\mathcal{U}}}\left(\mathbf{1}_{\lambda}\right)$ of $\dot{\mathcal{U}}$ (see section 6 for more details) is a module over the current algebra $\mathbf{U}\left(\mathfrak{s l}_{n}[t]\right)$. An immediate consequence of this result is the following theorem.

Theorem 1.3. Any 2-representation $\mathcal{U}\left(\mathfrak{s l}_{n}\right) \rightarrow \mathcal{K}$ gives rise to an action of the current algebra $\mathbf{U}\left(\mathfrak{s l}_{n}[t]\right)$ on $\operatorname{Tr}(\mathcal{K})$ and the center $Z(\mathcal{K})$ of objects in $\mathcal{K}$.

This theorem is proven in Section 6. Let us discuss one implication of this result. Brundan made the surprising discovery that one could define an action of the Lie algebra $\widehat{\mathfrak{g}}:=\mathfrak{g l}_{\infty}(\mathbb{C})$ on the center $Z(\mathcal{O})=$ $\bigoplus_{\nu} Z\left(\mathcal{O}_{\nu}\right)$ of all integral blocks $\mathcal{O}_{\nu}$ of category $\mathcal{O}[12$. In this action, the Chevalley generators of $\widehat{\mathfrak{g}}$ act as certain trace maps associated to canonical adjunction maps between special translation functors that arise from tensoring with a $\mathfrak{g}$-module and its dual. In [15] it is explained that this action is closely connected to Ginzburg's geometric construction of representations of the general linear group [25. Theorem 1.3 provides a context for understanding this surprising action by traces on category $\mathcal{O}$. Indeed, Brundan's action is part of a broad phenomenon that occurs within the context of higher representation theory: any categorified representation of the quantum group $\mathbf{U}_{q}\left(\mathfrak{s l}_{n}\right)$ immediately gives rise to an action of $\mathbf{U}\left(\mathfrak{s l}_{n}[t]\right)$ on the center of objects in the 2-representation via categorical traces. In particular, an action of $\dot{\mathcal{U}}\left(\mathfrak{s l}_{n}\right)$ on graded category $\mathcal{O}$ would automatically imply and generalize Brundan's result. More details about this action will be given in [7]. 
1.6.3. Organization of this paper. This paper is organized as follows. After recalling the diagrammatics for the trace in pivotal categories, taking as example Vect $_{k}$, we define the trace functor for linear and additive 1- and 2-categories and study its properties. In Section 3 we recall the notion of (strongly) upper-triangular category from 8 , which is particularly convenient for computing all HochschildMitchell homology groups. In the next sections different versions of the categorified quantum $\mathfrak{s l}_{n}$ are defined and their decategorifications by means of $K_{0}$ and $\mathrm{Tr}$ are compared. The last chapter is devoted to the proof of Theorem 1.3 .

To make this paper more accessible for graduate students, each section is supplied with many examples, exercises, pictures and references to textbooks. Places where special knowledge is required are marked and can be ignored in the first reading.

Acknowledgments: A.B. and Z.G. were supported by Swiss National Science Foundation under Grant PDFMP2-141752/1. K.H. was partially supported by JSPS Grant-in-Aid for Scientific Research (C) 24540077. A.D.L was partially supported by NSF grant DMS-1255334 and by the John Templeton Foundation. A.D.L is extremely grateful to Mikhail Khovanov for sharing his insights and vision about higher representation theory. Some of the ideas and calculations appearing in this article were done as part of this collaboration. He is also grateful to Arun Ram for helpful decategorification discussions. A.B. would like to thank Benjamin Cooper for helpful conversations.

\section{Elementary nOtions of trace}

2.1. Traces of linear maps. In this section we recall the definition and properties of the trace for any linear endomorphism. Throughout this paper $\mathbb{k}$ is assumed to be a field.

The trace of an $n \times n$ matrix $M=\left\{M_{i j}\right\}_{1 \leq i, j, \leq n}$ is the sum of its diagonal entries $\sum_{i} M_{i i}$. Given two square matrices $A, B$, the trace satisfies the trace property

$$
\operatorname{tr}(A B)=\operatorname{tr}(B A) .
$$

Exercise 2. Show that property (2.1) uniquely characterize the trace in the following sense: Two linear functions on the space of matrices satisfying (2.1) are proportional. Hint: any non-diagonal elementary matrix can be written as a commutator, i.e. $E_{i j}=\left[E_{i i}, E_{i j}\right]$.

More generally, for any finite-dimensional $\mathbb{k}$-vector space $V$, trace is a linear map

$$
\operatorname{tr}: \operatorname{End}_{\mathbb{k}}(V) \rightarrow \mathbb{k}
$$

such that the trace relation holds:

$$
\operatorname{tr}(f g)=\operatorname{tr}(g f) \quad \text { for any } f, g \in \operatorname{End}_{\mathbb{k}}(V) .
$$

Exercise 3. Show that this (basis independent) definition of the trace coincides with the usual one, where the trace of a matrix representing $f$ in some basis is taken. Hint: use Exercise 2 .

As a simple invariant of any linear endomorphism, the trace has a variety of important applications. Let us just mention few of them.

- For any representation $\phi: G \rightarrow \operatorname{Aut}_{\mathrm{k}}(V)$ of a group $G$, the character $\chi_{\phi}: G \rightarrow \mathbb{k}$ of the representation $\phi$ is the function sending each group element $g \in G$ to the trace of $\phi(g)$. The character of a representation carries much of the essential information about a representation. In particular, for $G$ a finite group and $\mathbb{k}=\mathbb{C}$, the complex representations of $G$ are determined up to isomorphism by their characters.

- A Lie algebra $\mathfrak{g}$ defined over $\mathbb{k}$ acts on itself via the adjoint action sending $x \in \mathfrak{g}$ to the endomorphism $\operatorname{ad}_{x}$ defined by $\operatorname{ad}_{x}(y)=[x, y]$ for all $y \in \mathfrak{g}$. When $\mathfrak{g}$ is finite dimensional, there is a symmetric bilinear form (or Killing form) $B: \mathfrak{g} \times \mathfrak{g} \rightarrow \mathbb{k}$ defined by $B(x, y):=\operatorname{tr}\left(\operatorname{ad}_{x} \operatorname{ad}_{y}\right.$ ). The non-degeneracy of this form is used to determine if a Lie algebra is semisimple. 
- In topology Markov trace is used to construct link invariants out of representations of braid groups.

2.1.1. Diagrammatics for the trace. The category $\mathbf{V e c t}_{\mathrm{k}}$ has the structure of a pivotal monoidal category. What this means is that this category admits a surprisingly rich diagrammatic calculus. In this calculus, edges are labelled by finite dimensional vector spaces and planar diagrams represent linear maps between the tensor product of vector spaces labelling the bottom strands to the tensor product of vector spaces labelling the top strands.

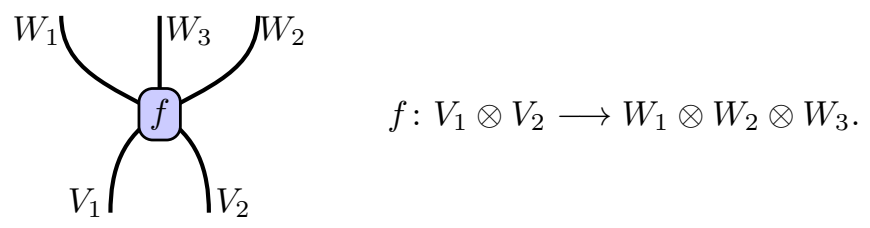

In this graphical calculus the ground field $\mathbb{k}$ is depicted by the empty strand. The ability to compose linear maps and to tensor linear maps is reflected in the graphical calculus by stacking the diagrams on top of each other and juxtaposing them side by side, respectively.

The "pivotal" structure on the category Vect $_{k}$ of finite-dimensional vector spaces indicates the existence of certain diagrams in the graphical calculus. To be concrete, fix a basis $\left\{e_{1}, \ldots, e_{n}\right\}$ of the vector space $V$. Denote by $\left\{e_{1}^{*}, \ldots, e_{n}^{*}\right\}$ the basis for the dual space $V^{*}=\operatorname{Hom}(V, \mathbb{k})$, with $e_{i}^{*}\left(e_{j}\right)=\delta_{i j}$. Then there are linear maps

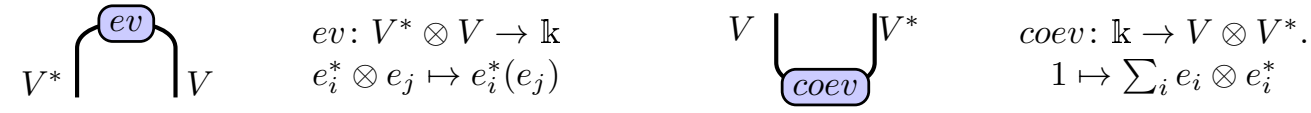

Exercise 4. Prove that the maps ev and coev satisfy the following identities

$$
\left(\operatorname{Id}_{V} \otimes e v\right) \circ\left(\operatorname{coev} \otimes \operatorname{Id}_{V}\right)=\operatorname{Id}_{V}, \quad \text { and } \quad\left(e v \otimes \operatorname{Id}_{V^{*}}\right) \circ\left(\operatorname{Id}_{V^{*}} \otimes \text { coev }\right)=\operatorname{Id}_{V^{*}}
$$

and depict these relations in the graphical calculus described above.

It is common practice in this diagrammatic calculus to simplify these diagrams

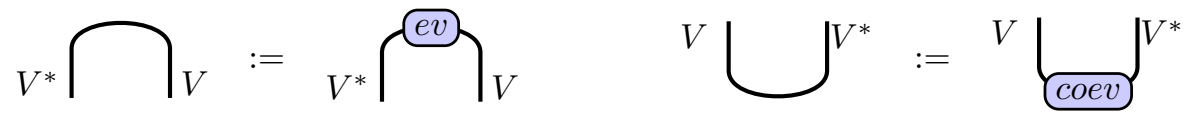

used to represent $e v$ and coev. Using the flip map in Vect $_{k}$

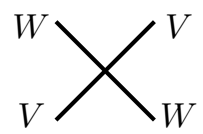

$$
\begin{aligned}
\tau: V \otimes W & \rightarrow W \otimes V \\
v \otimes w & \mapsto w \otimes v,
\end{aligned}
$$

we can also define other caps and caps
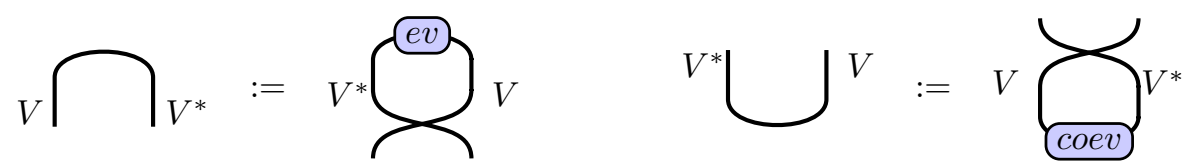

Exercise 5. Given a linear endomorphism $f: V \rightarrow V$ the following diagram

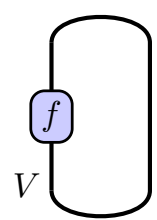


is multiplication by an element of the ground field $\mathbb{k}$. Show that this element is exactly the trace of $f$.

Utilizing the evaluation and coevaluation map it is possible to dualize any map $f: V \rightarrow W$ to get a map $f^{*}: W^{*} \rightarrow V^{*}$. Using these dual morphisms it is possible to slide linear maps through caps and cups
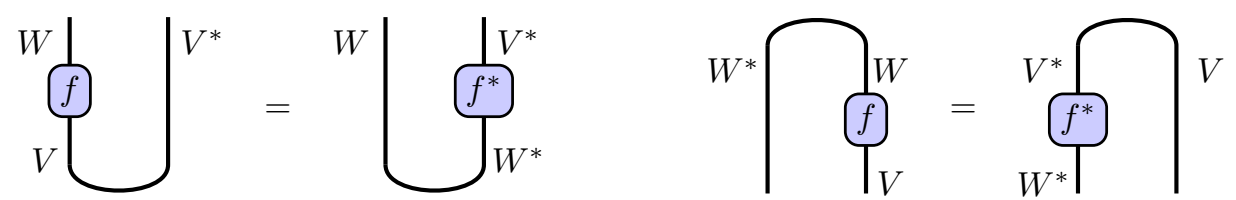

Using these facts and the identification of $\left(V^{*}\right)^{*}$ with $V$, it is not hard to verify the trace property:

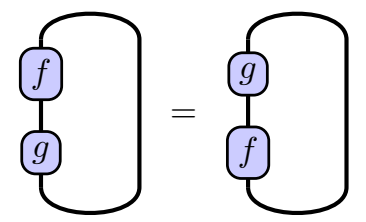

In particular, for $f=1_{V}$, the identity endomorphism of $V, \operatorname{tr}\left(1_{V}\right)=\operatorname{dim}(V)$. Here we are using the "right" trace. In a pivotal category one could also take the "left" trace and these two traces need not coincide.

This pictorial presentation was extended to the category of colored ribbon graphs, graphically describing arbitrary ribbon categories such as the category of finite dimensional representation of quantum groups [55, 60. This graphical calculus can also be seen as a specific instance of string diagrams in a 2-category [59, 26, 38].

2.2. Hochschild homology and the trace of a ring. In this section we will explain how the Hochschild homology of a ring $R$ can be viewed as "trace" of the ring $R$. Recall that for a ring $R$, the Hochschild homology $\mathrm{HH}_{*}(R)$ of $R$ can be defined as the homology of the Hochschild complex (see [50, 41]):

$$
C_{\bullet}=C_{\bullet}(R): \quad \ldots \longrightarrow C_{n} \stackrel{d_{n}}{\longrightarrow} C_{n-1} \stackrel{d_{n-1}}{\longrightarrow} \ldots \stackrel{d_{2}}{\longrightarrow} C_{1} \stackrel{d_{1}}{\longrightarrow} C_{0} \longrightarrow 0,
$$

where $C_{n}(R)=R^{\otimes n+1}$ and

$$
d_{n}\left(a_{0} \otimes \cdots \otimes a_{n}\right):=\sum_{i=0}^{n-1}(-1)^{i} a_{0} \otimes \cdots \otimes a_{i} a_{i+1} \otimes \cdots \otimes a_{n}+(-1)^{n} a_{n} a_{0} \otimes a_{1} \otimes \cdots \otimes a_{n-1}
$$

for $a_{0}, \ldots, a_{n-1} \in R$.

Exercise 6. Show that the zeroth Hochschild homology of the ring $R$ is isomorphic to the quotient of $R$ by the commutator subgroup,

$$
\mathrm{HH}_{0}(R) \cong R /[R, R]
$$

In particular, $\mathrm{HH}_{0}(R) \cong R$ for any commutative ring $R$.

Exercise 7. If $\operatorname{Mat}_{n}(R)$ denotes the ring of $n \times n$ dimensional matrices with coefficients in $R$, show that

$$
\mathrm{HH}_{0}\left(\operatorname{Mat}_{n}(R)\right) \cong \mathrm{HH}_{0}(R)
$$

Hint: consider exercise Q 
2.2.1. Diagrammatics for zeroth Hochschild homology. Many interesting rings $R$ can be represented by diagrams in the plane modulo some local relations. Multiplication in $R$ is represented by stacking diagrams on top of each other:

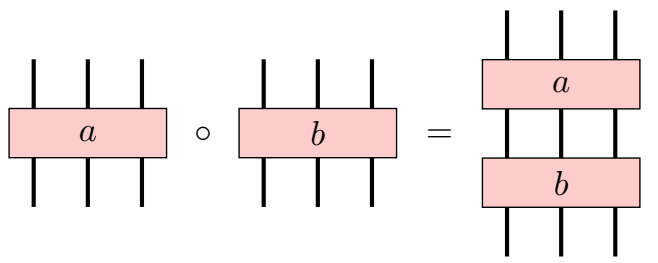

for $a, b \in R$. Then $\mathrm{HH}_{0}(R)$ can be described by diagrams on the annulus, modulo the same local relations as those for $R$ and the map

$$
R \longrightarrow R /[R, R]
$$

is given on diagrams by

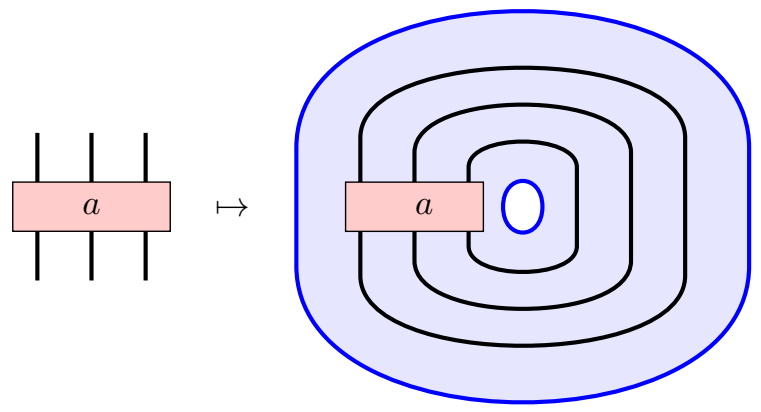

Notice that by sliding elements of $R$ around the annulus we see that $a b=b a$ in the quotient $\operatorname{HH}_{0}(R)$. Thus, the Hochschild homology of a ring $R$ exhibits similar properties to the trace of a linear map.

This method can be applied to Hecke algebras $H_{n, q}$ and their degenerations (0-Hecke algebras, nilCoxeter algebras) for a diagrammatic description of their Hochschild homology $\mathrm{HH}_{0}$ [11. In the next section we will use it to study $\mathrm{HH}_{0}$ of the nilHecke algebra.

2.2.2. Example calculations. The nilHecke ring $\mathrm{NH}_{n}$ is the graded unital associative ring generated by elements $x_{1}, \ldots, x_{n}$ of degree 2 and elements $\partial_{1}, \ldots, \partial_{n-1}$ of degree -2 , subject to the relations

$$
\begin{aligned}
& \partial_{i}^{2}=0, \quad \partial_{i} \partial_{i+1} \partial_{i}=\partial_{i+1} \partial_{i} \partial_{i+1} \\
& x_{i} \partial_{i}-\partial_{i} x_{i+1}=1, \quad \partial_{i} x_{i}-x_{i+1} \partial_{i}=1 \\
& x_{i} x_{j}=x_{j} x_{i} \quad(i \neq j), \quad \partial_{i} \partial_{j}=\partial_{j} \partial_{i} \quad(|i-j|>1), \\
& x_{i} \partial_{j}=\partial_{j} x_{i} \quad(|i-j|>1) .
\end{aligned}
$$

The ring $\mathrm{NH}_{n}$ has graphical presentation via $n$-stranded planar diagrams, generated by dots and crossings, modulo planar isotopies and certain local relations. In the graphical depiction of $\mathrm{NH}_{n}$ a dot on the $i$ th strand corresponds to a generator $x_{i}$, while a crossing of the $i$ and $i+1$ st strand represents $\partial_{i}$. Then we impose local relations
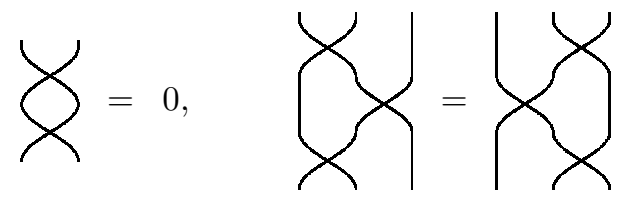


$$
1=\chi-\chi=\varnothing
$$

By [34, Section 2.5], the nilHecke ring $\mathrm{NH}_{n}$ is isomorphic to the $n ! \times n !$ matrix algebra $\operatorname{Mat}\left(n !, \operatorname{Sym}_{n}\right)$ with coefficients in the ring $\operatorname{Sym}_{n}$ of symmetric polynomials in $n$ variables,

$$
\operatorname{Sym}_{n} \cong \mathbb{Z}\left[x_{1}, \ldots, x_{n}\right]^{S_{n}} \text {. }
$$

Hence, by exercise 7 it follows that $\operatorname{Tr}\left(\mathrm{NH}_{n}\right) \cong \mathrm{Sym}_{n}$. The isomorphism between $\mathrm{NH}_{n}$ and the matrix algebra holds in the graded case, with $n$ ! replaced by the non-symmetric quantum factorial $(n)_{q^{2}}^{!}:=(n)_{q^{2}}(n-1)_{q^{2}} \ldots(1)_{q^{2}}$ where $(n)_{q^{2}}=\left(1-q^{2 n}\right) /\left(1-q^{2}\right)$, and with each variable of Sym $_{n}$ assigned degree 2 .

Let us illustrate the isomorphism $\operatorname{Tr}\left(\mathrm{NH}_{n}\right) \cong \mathrm{Sym}_{n}$ of graded abelian groups by some computations:

(1) The diagram

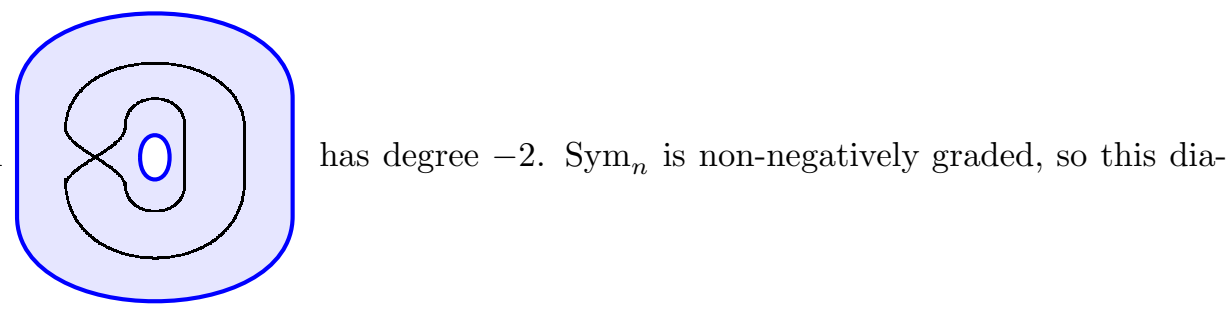
gram should be 0 in Tr. Indeed, we have

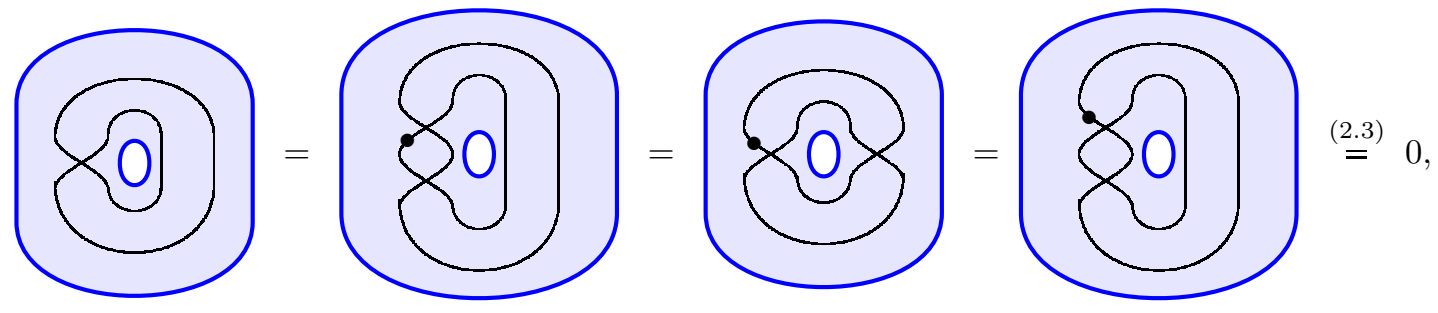

where the first equality follows from a combination of (2.4) and the first equality in (2.3).

(2) The degree 0 part of $\operatorname{Tr}\left(\mathrm{NH}_{2}\right)$ is spanned by
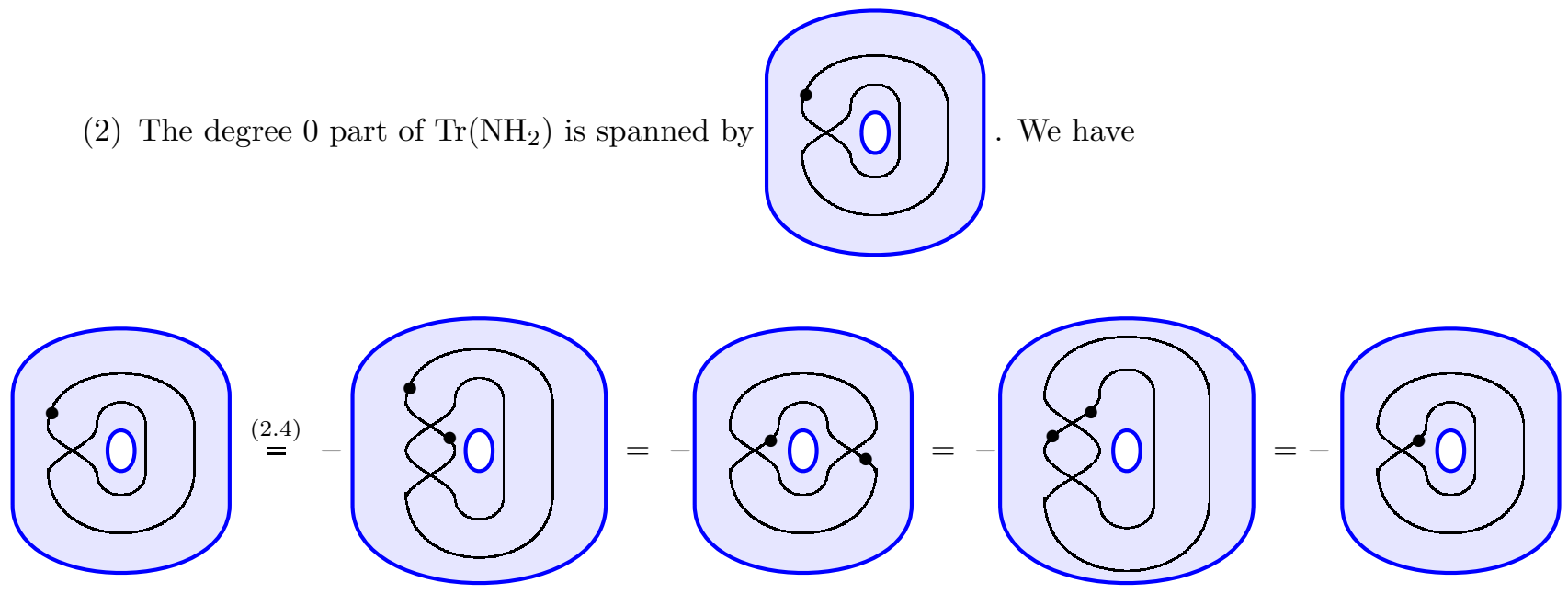
which implies

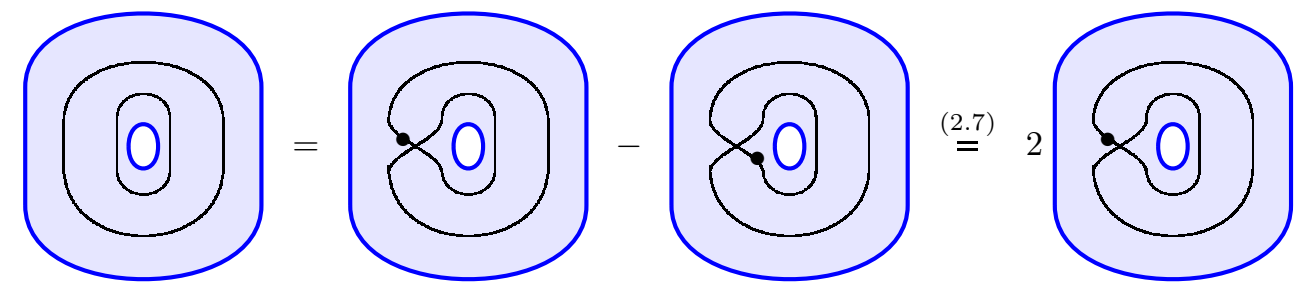

More generally, the element

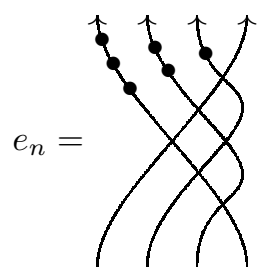

(shown for $n=4$ ) is a minimal idempotent in $\mathrm{NH}_{n}$. The composition

$$
\begin{aligned}
\operatorname{Sym}_{n} & \longrightarrow \mathrm{NH}_{n} \longrightarrow \operatorname{Tr}\left(\mathrm{NH}_{n}\right) \\
a \longmapsto a e_{n} & \longmapsto
\end{aligned}
$$

is a grading-preserving isomorphism of abelian groups. In particular, any basis of $\mathrm{Sym}_{n}$ gives a basis of $\operatorname{Tr}\left(\mathrm{NH}_{n}\right)$. Taking the basis $\left\{\varepsilon_{\lambda}\right\}_{\lambda}$ of $\operatorname{Sym}_{n}$, over all partitions with at most $n$ parts, $\lambda=\left(\lambda_{1}, \ldots, \lambda_{n}\right)$, $\lambda_{1} \geq \cdots \geq \lambda_{n}, \varepsilon_{\lambda}=\varepsilon_{\lambda_{1}} \ldots \varepsilon_{\lambda_{n}}, \varepsilon_{k}$ the $k$-th elementary symmetric function, gives us a basis of $\operatorname{Tr}\left(\mathrm{NH}_{n}\right)$.

Let $x^{\lambda}=x_{1}^{\lambda_{1}} \ldots x_{n}^{\lambda_{n}} \in \mathrm{NH}_{n}$.

Proposition 2.1 ( $[8])$. The image of $\left\{x^{\lambda} e_{n}\right\}_{\lambda}$ with $\lambda$ as above is a basis of $\operatorname{Tr}\left(\mathrm{NH}_{n}\right)$.

We call this basis the standard basis of $\operatorname{Tr}\left(\mathrm{NH}_{n}\right)$.

Problem 8. Determine the coefficients of inclusions $\mathrm{NH}_{n_{1}} \otimes \mathrm{NH}_{n_{2}} \stackrel{i}{\longrightarrow} \operatorname{Tr}\left(\mathrm{NH}_{n_{1}+n_{2}}\right)$ in the standard basis.

2.2.3. Zeroth Hochschild cohomology. The zeroth Hochschild cohomology $\operatorname{HH}^{0}(R)$ of a ring $R$ can be identified with the center of $R$,

$$
\mathrm{HH}^{0}(R) \cong Z(R)
$$

The Hochschild cohomology is naturally a module over the Hochschild homology. This action has a nice graphical interpretation as well. The action of $Z(R)$ on $\mathrm{HH}_{0}(R)$ can be graphically understood by cutting the diagram of an element $b \in \mathrm{HH}_{0}(R)$ along any ray emanating from the origin and inserting 
the diagram of $a \in Z(R)$ there:

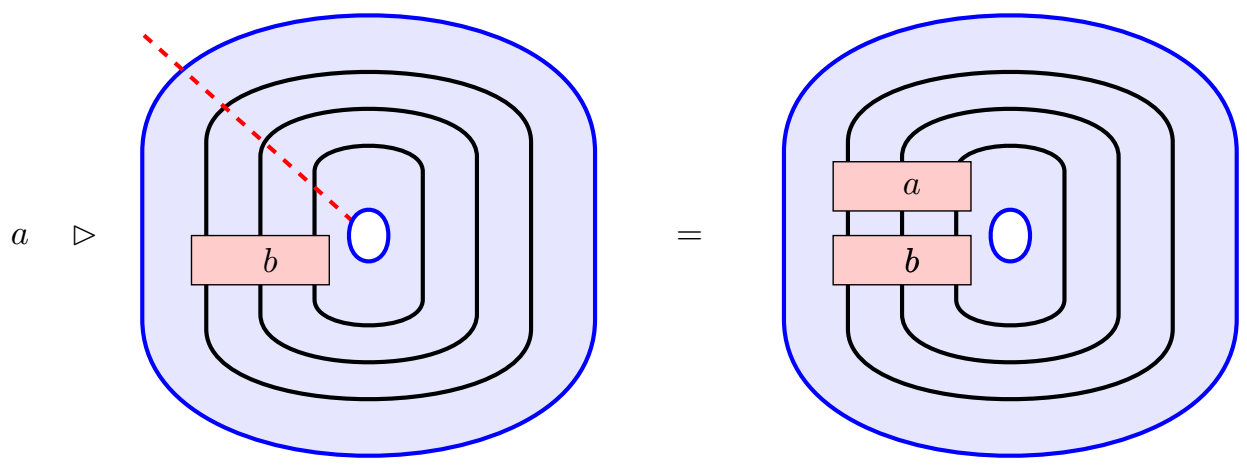

In section 6 we study an extension of this relationship between Hochschild homology and cohomology.

\section{TRACE OF A LINEAR CATEGORY}

In this section we define trace or zeroth Hochschild homology groups for linear and additive categories, and discuss their properties.

A category $\mathcal{C}$ is called linear if its hom-spaces are equipped with structures of abelian groups and the composition maps are bilinear. Such a structure is sometimes called an Ab-category, or a category enriched in the monoidal category $\mathbf{A b}$ of abelian groups. A linear functor between two linear categories $\mathcal{C}$ and $\mathcal{D}$ is a functor $F: \mathcal{C} \rightarrow \mathcal{D}$ such that for $x, y \in \mathrm{Ob}(\mathcal{C})$ the map $F: \mathcal{C}(x, y) \rightarrow \mathcal{D}(F(x), F(y))$ is an abelian group homomorphism.

Example 1. The Temperley-Lieb category TL is defined as a quotient of the $\mathbb{Z}\left[A, A^{-1}\right]$-span of tangles by the Kauffman bracket skein relations:

$$
\begin{aligned}
& \left.\langle\searrow /\rangle=A\langle)(\rangle+A^{-1}\langle\rangle\right\rangle, \\
& \langle T \amalg \bigcirc\rangle=\left(-A^{-2}-A^{2}\right)\langle T\rangle .
\end{aligned}
$$

The objects of this category are natural numbers corresponding to the boundary points of the tangle. A morphism in $\mathrm{TL}$ is a $\mathbb{Z}\left[A, A^{-1}\right]$-linear combination of crossingless matchings between the boundary points.

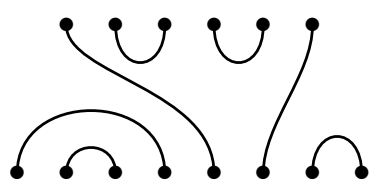

3.1. Hochschild-Mitchell homology and the trace. Any ring $R$ with unit $1_{R}$ can be viewed as a linear category with one object $*$ and whose morphisms $\operatorname{Hom}(*, *)$ are given by the abelian group $R$. Composition is given by multiplication in $R$ and the identity morphisms of $*$ corresponds to the unit $1_{R} \in R$. Utilizing this observation, it possible to define the Hochschild-Mitchell homology of an arbitrary linear category as follows.

Let $\mathcal{C}$ be a small linear category. Define the Hochschild-Mitchell complex of $\mathcal{C}$

$$
C_{\bullet}=C_{\bullet}(\mathcal{C}): \quad \ldots \longrightarrow C_{n} \stackrel{d_{n}}{\longrightarrow} C_{n-1} \stackrel{d_{n-1}}{\longrightarrow} \ldots \stackrel{d_{2}}{\longrightarrow} C_{1} \stackrel{d_{1}}{\longrightarrow} C_{0} \longrightarrow 0,
$$


where

$$
\begin{gathered}
C_{n}=C_{n}(\mathcal{C}):=\bigoplus_{x_{0}, \ldots, x_{n} \in \operatorname{Ob}(\mathcal{C})} \mathcal{C}\left(x_{n}, x_{0}\right) \otimes \mathcal{C}\left(x_{n-1}, x_{n}\right) \otimes \cdots \otimes \mathcal{C}\left(x_{0}, x_{1}\right), \\
d_{n}\left(\sigma_{n} \otimes \sigma_{n-1} \otimes \cdots \otimes \sigma_{0}\right):=\sum_{i=0}^{n-1}(-1)^{i} \sigma_{n} \otimes \cdots \otimes \sigma_{n-i} \sigma_{n-i-1} \otimes \cdots \otimes \sigma_{0}+(-1)^{n} \sigma_{0} \sigma_{n} \otimes \sigma_{n-1} \otimes \cdots \otimes \sigma_{1} .
\end{gathered}
$$

The Hochschild-Mitchell homology $\mathrm{HH}_{\bullet}(\mathcal{C})$ of $\mathcal{C}$ is defined to be the homology of the chain complex $C$. $(\mathcal{C})$. One can easily check that if $\mathcal{C}$ is a unital ring $R$ regarded as a linear category, then this definition agrees with the one given in section 2.2 .

In this article we will be especially concerned with the zeroth Hochschild-Mitchell homology $\operatorname{HH}_{0}(\mathcal{C})=$ $C_{0} / d_{1}\left(C_{1}\right)$ of $\mathcal{C}$. We introduce the notation

$$
\operatorname{Tr}(\mathcal{C}):=\mathrm{HH}_{0}(\mathcal{C})
$$

which we call the trace of the linear category $\mathcal{C}$. This terminology is justified by the following observation. For any $x \in \operatorname{Ob}(\mathcal{C})$ we denote by $\mathcal{C}(x, x)=\operatorname{Hom}_{\mathcal{C}}(x, x)=\operatorname{End}_{\mathcal{C}}(x)$ the space of its endomorphisms. Then it is an easy calculation to verify that the trace $\operatorname{Tr}(\mathcal{C})$ of the linear category $\mathcal{C}$ is given by

$$
\operatorname{Tr}(\mathcal{C})=\left(\bigoplus_{x \in \operatorname{Ob}(\mathcal{C})} \mathcal{C}(x, x)\right) / \operatorname{Span}\{f g-g f\},
$$

where $f$ and $g$ run through all pairs of morphisms $f: x \longrightarrow y, g: y \longrightarrow x$ with $x, y \in \operatorname{Ob}(\mathcal{C})$.

We denote by $[f]$ the equivalence class of $f \in \mathcal{C}(x, x)$ in $\operatorname{Tr}(\mathcal{C})$. The trace $\operatorname{Tr}$ gives rise to a functor from the category of (small) linear categories to the category of abelian groups.

3.2. The trace and direct sums. In this section we show that the trace behaves well with respect to direct sums in additive categories. An additive category is a linear category equipped with a zero object and biproducts, also called direct sums.

Lemma 3.1. If $\mathcal{C}$ is an additive category, then for $f: x \rightarrow x$ and $g: y \rightarrow y$, we have

$$
[f \oplus g]=[f]+[g]
$$

in $\operatorname{Tr}(\mathcal{C})$.

Proof. Since $f \oplus g=(f \oplus 0)+(0 \oplus g): x \oplus y \rightarrow x \oplus y$, we have

$$
[f \oplus g]=[f \oplus 0]+[0 \oplus g]
$$

Now we have $[f \oplus 0]=[i f p]=[p i f]=[f]$ where $p: x \oplus y \rightarrow x$ and $i: x \rightarrow x \oplus y$ are the projection and the inclusion. Similarly, we have $[0 \oplus g]=[g]$. Hence the result.

A linear (resp. additive) category enriched over Vect $_{\mathbb{k}}$ will be called $\mathbb{k}$-linear, i.e. its homs are $\mathbb{k}$-linear vector spaces and composition is $\mathbb{k}$-bilinear.

Example 2. Let $\mathcal{C}=$ Vect $_{\mathbb{k}}$ be the $\mathbb{k}$-linear category of finite dimensional $\mathbb{k}$-vector spaces. Since any finite dimensional vector space is isomorphic to a finite direct sum of copies of $\mathbb{k}$, by Lemma 3.1 we have $\operatorname{Tr}\left(\operatorname{Vect}_{\mathrm{k}}\right)=\mathbb{k}$.

Example 3. Let $\mathcal{C}=$ gVect $_{\mathbb{k}}$ be the $\mathbb{k}$-linear category of finite dimensional $\mathbb{Z}$-graded vector spaces, i.e. any $V \in \operatorname{Ob}(\mathcal{C})$ decomposes as $V=\bigoplus_{n \in \mathbb{Z}} V_{n}$ with $\operatorname{deg}(x)=n$ for any $x \in V_{n}$ and morphisms in $\mathbf{g V e c t}_{\mathbb{k}}$ are degree preserving. Then $\operatorname{Tr}\left(\mathbf{g V e c t}_{\mathrm{k}}\right)=\bigoplus_{n \in \mathbb{Z}} \mathbb{k}=\mathbb{k}\left[q, q^{-1}\right]$. The multiplication by $q$ is interpreted as a shift of degree by one. 
The previous examples can be further generalized. For a linear category $\mathcal{C}$, there is a "universal" additive category generated by $\mathcal{C}$, called the additive closure $\mathcal{C}^{\oplus}$, in which the objects are formal finite direct sums of objects in $\mathcal{C}$ and the morphisms are matrices of morphisms in $\mathcal{C}$. There is a canonical fully faithful functor $\mathcal{C} \rightarrow \mathcal{C}^{\oplus}$. Every linear functor $F: \mathcal{C} \rightarrow \mathcal{D}$ to an additive category $\mathcal{D}$ factors through it uniquely up to natural isomorphism.

Exercise 9. Show that for a linear category $\mathcal{C}, \operatorname{Tr}\left(\mathcal{C}^{\oplus}\right)=\operatorname{Tr}(\mathcal{C})$. Hint: Hom-spaces of $\mathcal{C}^{\oplus}$ are modules consisting of matrices whose entries are morphisms in $\mathcal{C}$.

Exercise 10. Show that $\operatorname{Tr}(\mathrm{TL})=\mathbb{Z}\left[A, A^{-1}\right][z]$, where $[z]=\left[1_{1}\right]$. Hint: $\operatorname{Tr}(\mathrm{TL})$ can be naturally identified with the Kauffman skein module of the solid torus by sending $\left[1_{1}\right]$ to the non-contractible circle.

3.3. The trace and idempotent completions. A projection or idempotent in Vect $_{\mathrm{k}}$ is an endomorphism $p: V \rightarrow V$ satisfying the relation $p^{2}=p$. In this case, $\left(\operatorname{Id}_{V}-p\right): V \rightarrow V$ is also an idempotent and together these two idempotents decompose the space $V$ into a direct sum of the image of the projection $p$ and the image of the projection $\operatorname{Id}_{V}-p$.

More generally, idempotents can be defined in any category $\mathcal{C}$ as endomorphisms $e: x \rightarrow x$ satisfying $e^{2}=e$, but here we will always assume that $\mathcal{C}$ is a linear category.

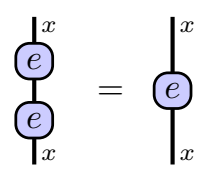

An idempotent $e: x \rightarrow x$ in $\mathcal{C}$ is said to split if there is an object $y$ and morphisms $g: x \rightarrow y, h: y \rightarrow x$ such that $h g=e$ and $g h=1_{y}$.

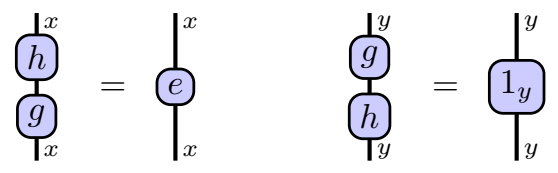

Unfortunately, even in an additive category $\mathcal{C}$ it is not always possible to split idempotents. Such phenomenon occurs quite often when studying additive categories $\mathcal{C}$ defined diagrammatically by generators and relations [36, 51, [5]. In this case, making sense out of the "image" of a diagram is not usually possible. A common solution is to enlarge the category $\mathcal{C}$ by passing to the Karoubi envelope $\operatorname{Kar}(\mathcal{C})$ (also called idempotent or Cauchy completion) of $\mathcal{C}$. The Karoubi envelope can be thought of as a minimal enlargement of the category $\mathcal{C}$ in which all idempotents split.

More formally, the Karoubi envelope $\operatorname{Kar}(\mathcal{C})$ is the category whose objects are pairs $(x, e)$ of objects $x \in \mathrm{Ob}(\mathcal{C})$ and an idempotent endomorphism $e: x \rightarrow x$ in $\mathcal{C}$. The morphisms

$$
f:(x, e) \rightarrow\left(y, e^{\prime}\right)
$$

are morphisms $f: x \rightarrow y$ in $\mathcal{C}$ such that $f=e^{\prime} f e$, or alternatively such that

$$
\underbrace{y}_{x}=\overbrace{e_{x}}^{e^{y}} .
$$

Composition is induced by the composition in $\mathcal{C}$ and the identity morphism is $e:(x, e) \rightarrow(x, e) . \operatorname{Kar}(\mathcal{C})$ is equipped with a linear category structure. 
We can identify $\left(x, 1_{x}\right)$ with the object $x$ of $\mathcal{C}$. This identification gives rise to a natural embedding functor $\iota: \mathcal{C} \rightarrow \operatorname{Kar}(\mathcal{C})$ such that $\iota(x)=\left(x, 1_{x}\right)$ for $x \in \operatorname{Ob}(\mathcal{C})$ and $\iota(f: x \rightarrow y)=f$. The Karoubi envelope $\operatorname{Kar}(\mathcal{C})$ has the universality property that if $F: \mathcal{C} \rightarrow \mathcal{D}$ is a linear functor to a linear category $\mathcal{D}$ with split idempotents, then $F$ extends to a functor from $\operatorname{Kar}(\mathcal{C})$ to $\mathcal{D}$ uniquely up to natural isomorphism [10, Proposition 6.5.9].

Example 4. Consider the Temperley-Lieb category TL defined over $\mathbb{C}(q)$, where $q$ is an indeterminant with $q=-A^{2}$. The Karoubi envelope $\operatorname{Kar}(\mathrm{TL})$ is equivalent as a category to the category of finite-dimensional representations of quantum $\mathfrak{s l}_{2}$ ([29] and [53]). The irreducible $N$-dimensional representation arises as the image of a certain idempotent called Jones-Wenzl idempotent.

Exercise 11. Show that the Karoubi envelope of an additive category is additive.

The following proposition illustrates one of the key advantages of the trace, namely its invariance under passing to the Karoubi envelope.

Proposition 3.2. The map $\operatorname{Tr}(\iota): \operatorname{Tr}(\mathcal{C}) \longrightarrow \operatorname{Tr}(\operatorname{Kar}(\mathcal{C}))$ induced by $\iota$ is bijective.

Proof. Recall that an endomorphism $f:(x, e) \rightarrow(x, e)$ in $\operatorname{Kar}(\mathcal{C})$ is just a morphism $f: x \rightarrow x$ in $\mathcal{C}$ satisfying the condition that $f=e f e$. Define a map $u: \operatorname{Tr}(\operatorname{Kar}(\mathcal{C})) \longrightarrow \operatorname{Tr}(\mathcal{C})$ sending $[f] \in \operatorname{Tr}(\operatorname{Kar}(\mathcal{C}))$ to $[f] \in \operatorname{Tr}(\mathcal{C})$. Then one can check that $u$ is an inverse to $\operatorname{Tr}(\iota)$.

Example 5. Adding Jones-Wenzl idempotents as objects into TL does not affect $\operatorname{Tr}(\mathrm{TL})$.

3.4. The trace and categories of complexes. The trace of a linear category $\mathcal{C}$ gives rise to an interesting variant of Euler characteristic. Let $\operatorname{Kom}(\mathcal{C})$ be the category of bounded complexes over $\mathcal{C}$. Recall that the objects of $\operatorname{Kom}(\mathcal{C})$ are complexes

$$
A_{\bullet}: \quad \ldots \longrightarrow A_{i} \stackrel{d_{i}}{\longrightarrow} A_{i-1} \stackrel{d_{i-1}}{\longrightarrow} \ldots \stackrel{d_{2}}{\longrightarrow} A_{1} \stackrel{d_{1}}{\longrightarrow} A_{0} \longrightarrow 0,
$$

where $A_{i} \in \mathrm{Ob}(\mathcal{C})$ and $d_{i} \in \mathcal{C}\left(A_{i}, A_{i-1}\right)$, and the morphisms are chain maps.

The "traced" Euler characteristic of a complex $A_{\bullet}$ is defined by

$$
\chi_{\operatorname{tr}}\left(A_{\bullet}\right)=\sum_{i}(-1)^{i}\left[1_{A_{i}}\right] \in \operatorname{Tr}(\mathcal{C}),
$$

where $\left[1_{x}\right]$ is the class of $1_{x}$ in $\operatorname{Tr}(\mathcal{C})$ for any $x \in \operatorname{Ob}(\mathcal{C})$. More generally, to any chain endomorphism $F: A_{\bullet} \rightarrow A_{\bullet}$ we can associate its "traced" Lefschetz number

$$
\tau(F)=\sum_{i}(-1)^{i}\left[F_{i}\right]
$$

Then $\chi_{t r}\left(A_{\bullet}\right)=\tau\left(1_{A_{\bullet}}\right)$.

Lemma 3.3. For any $A_{\bullet} \in \operatorname{Kom}(\mathcal{C})$ and homotopic chain endomorphisms $F, G: A_{\bullet} \rightarrow A_{\bullet}$ we have $\tau(F)=\tau(G)$. Furthermore, $\chi_{\text {tr }}$ is homotopy invariant.

Exercise 12. Prove this lemma using the definition of the chain homotopy, i.e. $F-G=d h+h d$.

An interesting application of the traced Euler characteristic was given by Bar-Natan [4]. There he defined a quotient $\mathrm{Cob}$ / of the $\mathbb{Z}[1 / 2]$-linear category of 2-dimensional cobordisms, and associated a complex in $\operatorname{Kom}\left(\mathrm{Cob}_{/}^{\oplus}\right)$ to any tangle, which he called a Khovanov bracket. He showed that isotopies of the tangle do not change the homotopy type of this complex. The following theorem of Bar-Natan shows that the traced Euler characteristic can be used as decategorification map in a categorification of the Jones polynomial.

Theorem 3.4 (Theorem 6 [4]). The traced Euler characteristic of the Khovanov bracket of a tangle in Bar-Natan's category is equal to the Jones polynomial. 
3.5. The trace and split Grothendieck groups. For a small additive category $\mathcal{C}$, the split Grothendieck group $K_{0}(\mathcal{C})$ of $\mathcal{C}$ is the abelian group generated by the isomorphism classes of objects of $\mathcal{C}$ with relations $[x \oplus y]_{\cong}=[x]_{\cong}+[y]_{\cong}$ for $x, y \in \mathrm{Ob}(\mathcal{C})$. Here $[x] \cong$ denotes the isomorphism class of $x$. The split Grothendieck group $K_{0}$ defines a functor from additive categories to abelian groups. Here the morphisms in the category of additive categories are additive functors.

Example 6. $K_{0}\left(\right.$ Vect $\left._{\mathrm{k}}\right)=\mathbb{Z}$ is generated by $[\mathbb{k}] \cong$.

More generally, for any commutative ring $R$, let $\mathrm{FMod}_{\mathrm{R}}$ be the additive category of finitely generated free $R$-modules. Then $K_{0}\left(\mathrm{FMod}_{\mathrm{R}}\right)=\mathbb{Z}$ with the isomorphism given by rank. The split Grothendieck group $K_{0}$ of the additive category of finitely generated projective $R$-modules is generated by the indecomposable projective modules. Over a local ring, any finitely generated projective module is free, so up to isomorphism there is a unique indecomposable projective module, see for example [49, Chapter 1] or [56].

In $\operatorname{Kom}(\mathcal{C})$ the Euler characteristic of a complex $A$ defined by

$$
\chi\left(A_{\bullet}\right)=\sum_{i}(-1)^{i} K_{0}\left(A_{i}\right)=\sum_{i}(-1)^{i}\left[A_{i}\right] \cong
$$

is homotopy invariant.

3.5.1. Generalized Chern character. Classically, the Chern character is a homomorphism of rings from the topological $K$-theory of a manifold into the de Rham cohomology of the manifold. Given a vector bundle on a manifold $M$, the Chern character map produces an element in the de Rham cohomology of the manifold. In the algebraic framework, a commutative associative algebra $A$ plays the role of the manifold $M$, and a vector bundle in topological $K$-theory is replaced by a finitely-generated projective module over $A$ in algebraic $K$-theory. Passing to the non-commutative setting where $A$ is no longer assumed commutative, the Chern character map can still be defined, giving a homomorphism from $K_{0}(A)$ into the cyclic homology of $A$ (see chapter 8 of [41). The zeroth cyclic homology $\mathrm{HC}_{0}(A)$ is isomorphic to the zeroth Hochschild homology $\operatorname{HH}_{0}(A)$ [41, Section 2.1.12], so we can view the Chern character as a map from $K_{0}(A) \rightarrow \mathrm{HH}_{0}(A)$. In this section we study a further generalization of the Chern character map.

Define a homomorphism

$$
h_{\mathcal{C}}: K_{0}(\mathcal{C}) \longrightarrow \operatorname{Tr}(\mathcal{C})
$$

by

$$
h_{\mathcal{C}}([x] \cong)=\left[1_{x}\right]
$$

for $x \in \mathrm{Ob}(\mathcal{C})$. Indeed, one can easily check that

$$
h_{\mathcal{C}}\left([x \oplus y]_{\cong}\right)=\left[1_{x}\right]+\left[1_{y}\right],
$$

since $1_{f \oplus g}=1_{f} \oplus 1_{g}$. The map $h_{\mathcal{C}}$ defines a natural transformation

$$
h: K_{0} \Rightarrow \operatorname{Tr}: \mathbf{A d C a t} \rightarrow \mathbf{A b},
$$

where AdCat denotes the category of additive small categories.

Example 7. In Example 8.3.6 of [41, it is shown that for an associative unital algebra $A$ the map $h: K_{0}(A) \rightarrow \mathrm{HH}_{0}(A)=A /[A, A]$ is an isomorphism whenever $A$ is a field, a local ring, or $\mathbb{Z}$.

We illustrate below that $h_{\mathcal{C}}$ is neither injective nor surjective in general.

Example 8. Let $F_{p}=\mathbb{Z} / p \mathbb{Z}$ denote the field with $p$ elements for $p$ prime. If $\mathcal{C}$ is the category of finite dimensional $F_{p}$-vector spaces, then $h_{\mathcal{C}}$ maps $K_{0}(\mathcal{C})=\mathbb{Z}$ surjectively onto $\operatorname{Tr}(\mathcal{C})=F_{p}$, but it is not injective. 
Since $\operatorname{Tr}$ is always linear over the ground ring we are using, and $K_{0}$ is $\mathbb{Z}$-linear, one can wonder whether they are isomorphic after tensoring $K_{0}$ with the ground ring. Computations for the nilHecke ring and its quotients illustrate that this is not true in general.

Example 9. We showed earlier that $\operatorname{Tr}\left(\mathrm{NH}_{n}\right)=\operatorname{Tr}\left(\mathrm{Sym}_{n}\right)=\mathrm{Sym}_{n}$. However, $K_{0}\left(\mathrm{NH}_{n}\right) \cong \mathbb{Z}$ so the map

$$
h_{\mathrm{NH}_{n}}: K_{0}\left(\mathrm{NH}_{n}\right)=\mathbb{Z} \longrightarrow \operatorname{Sym}_{n}=\operatorname{Tr}\left(\mathrm{NH}_{n}\right)
$$

is clearly not surjective.

Example 10. For any integer $0 \leq k \leq n$ define cyclotomic quotients of the nilHecke algebra $\mathrm{NH}_{n}^{k}$ as the quotient $\mathrm{NH}_{n} /\left(x_{1}\right)^{k}$. Taken together over all $k$, these cyclotomic quotients give rise to categorifications of irreducible representations of $\mathbf{U}_{q}\left(\mathfrak{s l}_{2}\right)$ [13, 14, 38, 39, 62]. In [38, Proposition 5.3] it is shown that

$$
\mathrm{NH}_{n}^{k} \cong \operatorname{Mat}\left((n)_{q^{2}}^{!} ; H^{*}(\operatorname{Gr}(k, n))\right) \text {, }
$$

where $H^{*}(\operatorname{Gr}(k, n))$ denotes the graded cohomology ring of the Grassmannian of $k$-dimensional planes in complex $n$-dimensional space. This ring has an explicit description as the quotient of the graded polynomial ring $\mathbb{Z}\left[c_{1}, \ldots, c_{k} ; \bar{c}_{1}, \ldots, \bar{c}_{N-k}\right]$ with $\operatorname{deg} c_{j}=2 j, \operatorname{deg} \bar{c}_{j}=2 j$ by the homogeneous ideal generated by the elements $\sum c_{j} \bar{c}_{\alpha-j}$ for $\alpha>0$. Note that we set $c_{0}=\bar{c}_{0}=1, c_{j}=0$ for $j<0$ or $j>k$, and $\bar{c}_{\ell}=0$ for $\ell<0$ and $\ell>N-k$.

Since the cohomology ring $H^{*}(\operatorname{Gr}(k, n))$ is graded local, $K_{0}\left(H^{*}(\operatorname{Gr}(k, n))\right) \cong \mathbb{Z}$, but since $H^{*}(\operatorname{Gr}(k, n))$ is commutative, by exercise 7 we have $\operatorname{Tr}\left(\mathrm{NH}_{n}^{k}\right)=\operatorname{Tr}\left(H^{*}(\operatorname{Gr}(k, n))\right)=H^{*}(\operatorname{Gr}(k, n))$, so that the homomorphism

is also not surjective.

$$
h_{\mathrm{NH}_{n}^{k}}: K_{0}\left(\mathrm{NH}_{n}^{k}\right) \cong \mathbb{Z} \longrightarrow H^{*}(\operatorname{Gr}(k, n))=\operatorname{Tr}\left(\mathrm{NH}_{n}^{k}\right)
$$

3.6. The trace of a graded linear category. A graded linear category is a category equipped with an auto-equivalence $\langle 1\rangle$. We denote by $\langle t\rangle$ the auto-equivalence obtained by applying $\langle 1\rangle t$ times. Thus, given any object $x \in \mathrm{Ob}(\mathcal{C})$, there exists an object $x\langle t\rangle \in \mathrm{Ob}(\mathcal{C})$ for all $t$. Likewise, given any morphism $f: x \rightarrow y$ in $\mathcal{C}$, there is a morphism $f\langle t\rangle: x\langle t\rangle \rightarrow y\langle t\rangle$. This equips Hom-sets with the structure of $\mathbb{Z}$-graded abelian groups in which composition maps are grading preserving.

Given a graded linear category $\mathcal{C}$, its Grothendieck group $K_{0}(\mathcal{C})$ is a $\mathbb{Z}\left[q, q^{-1}\right]$-module with $[x\langle t\rangle] \cong:=$ $q^{t}[x]$. Likewise, the trace $\operatorname{Tr}(\mathcal{C})$ is $\mathbb{Z}\left[q, q^{-1}\right]$-module with $[f\langle t\rangle]=q^{t}[f]$ for an endomorphism $f: x \rightarrow x$ in $\mathcal{C}$. In this case, the generalized Chern character map

$$
K_{0}(\mathcal{C}) \rightarrow \operatorname{Tr}(\mathcal{C})
$$

is a homomorphism of $\mathbb{Z}\left[q, q^{-1}\right]$-modules.

A graded linear category $\mathcal{C}$ is said to admit a translation if for every object $x$ in $\mathcal{C}$ and integer $t \in \mathbb{Z}$, there is an isomorphism $x \rightarrow x\langle t\rangle$. Given any graded linear category $\mathcal{C}$, we can form a graded linear category $\mathcal{C}^{*}$ that admits translations by declaring that $\mathrm{Ob}(\mathcal{C})=\mathrm{Ob}\left(\mathcal{C}^{*}\right)$ and setting

$$
\mathcal{C}^{*}(x, y):=\bigoplus_{t \in \mathbb{Z}} \mathcal{C}(x, y\langle t\rangle)
$$

for all objects $x, y \in \mathrm{Ob}(\mathcal{C})$. The translations $x \rightarrow x\langle t\rangle$ is given by $1_{x}$ in $\mathcal{C}^{*}$ since

$$
1_{x} \in \mathcal{C}(x, x\langle 0\rangle)=\mathcal{C}(x,(x\langle t\rangle)\langle-t\rangle) \subset \mathcal{C}^{*}(x, x\langle t\rangle),
$$

has inverse

$$
1_{x}\langle t\rangle \in \mathcal{C}(x\langle t\rangle, x\langle t\rangle)=\mathcal{C}(x\langle t\rangle,(x\langle 0\rangle)\langle t\rangle) \subset \mathcal{C}^{*}(x\langle t\rangle, x) .
$$

By enlarging the hom spaces by adding isomorphisms $x \rightarrow x\langle t\rangle$, the Grothendieck group of graded linear category with translation $\mathcal{C}^{*}$ is only a $\mathbb{Z}$-module rather than a $\mathbb{Z}\left[q, q^{-1}\right]$-module since $[x\langle t\rangle] \cong=$ $[x] \cong$. However, the trace $\operatorname{Tr}\left(\mathcal{C}^{*}\right)$ changes more drastically because, rather than only considering degree 
preserving endomorphisms $f: x \rightarrow x$ in $\mathcal{C}$, we can now consider arbitrary degree maps $x \rightarrow x\langle t\rangle$ and compose with the translation map from $x\langle t\rangle \rightarrow x$ to get a new endomorphism. Furthermore, using the translations $x \cong x\langle t\rangle$, it is not hard to see that given any endomorphism $f: x \rightarrow x$, we have $[f\langle t\rangle]=[f]$ in $\operatorname{Tr}\left(\mathcal{C}^{*}\right)$, so that the graded version of the trace $\operatorname{Tr}\left(\mathcal{C}^{*}\right)$ is also just a $\mathbb{Z}$-module.

As explained at the beginning of section 3.1, any ring with unit can be viewed as a linear category with one object $*$. A graded ring $R=\oplus_{n \in \mathbb{Z}} R_{n}$ can be viewed as a graded category whose objects are indexed by the integers $n=*\langle n\rangle$ for $n \in \mathbb{Z}$. The morphisms from $n$ to $m$ are given by the abelian group $R_{m-n}$. Composition is given by the grading preserving multiplication in $R$. Example 7 can be generalized to show that the generalized Chern character map $K_{0}(R) \rightarrow \operatorname{Tr}\left(R_{0}\right)$ is an isomorphism whenever $R$ is a graded local ring, so that the trace of the degree zero part of the ring is isomorphic to the Grothendieck ring.

Exercise 13. If $\mathcal{C}$ denotes the graded linear category associated to a graded ring $R$, then the graded linear category with translation $\mathcal{C}^{*}$ is equivalent as a category to the linear category with one object $*$ obtained by forgetting the grading on $R$.

Example 11. The nilHecke algebra $\mathrm{NH}_{n}$ is a graded algebra with $\operatorname{deg}\left(x_{i}\right)=2$ and $\operatorname{deg}\left(\partial_{i}\right)=-2$. Recall that the nilHecke ring $\mathrm{NH}_{n}$ is isomorphic as a graded algebra to the matrix $\operatorname{ring} \operatorname{Mat}\left((n)_{q^{2}}^{!} ; \operatorname{Sym}_{n}\right)$ of $n ! \times n !$ matrices with coefficients in the ring $\operatorname{Sym}_{n}=\mathbb{Z}\left[x_{1}, \ldots, x_{n}\right]^{S_{n}}$ of symmetric functions in $n$ variables. Here we write $(n)_{q^{2}}:=\left(1-q^{2 n}\right) /\left(1-q^{2}\right)$ for the non-symmetrized quantum integers and each variable of $\operatorname{Sym}_{n}$ has degree 2 .

To compute the trace of $\mathrm{NH}_{n}$ regarded as a graded category $\mathcal{C}$, observe that $\operatorname{Tr}(\mathcal{C})=\operatorname{Tr}(\mathrm{Sym})$ and that Sym is positively graded and one dimensional in degree zero, in particular it is graded local, so that the graded trace of $\operatorname{Tr}(\mathcal{C}) \cong K_{0}\left(\mathrm{NH}_{n}\right) \cong \mathbb{Z}$. Compare this result with section 2.2 .2 where we showed $\operatorname{Tr}\left(\mathrm{NH}_{n}\right) \cong \operatorname{Sym}_{n}$. Exercise 13 shows that by enlarging the graded category $\mathcal{C}$ to $\mathcal{C}^{*}$, we again have $\operatorname{Tr}\left(\mathcal{C}^{*}\right)=\operatorname{Sym}_{n}$.

3.7. Hochschild-Mitchell homology of strongly upper triangular categories. Recall that the trace of a linear category is just the zeroth Hochschild-Mitchell homology. There is a class of linear categories for which we can compute explicitly their Hochschild-Mitchell homology. The results developed in this section will be useful in our study of the Hochschild-Mitchell homology of categorified quantum groups.

Definition 3.5. A linear category $\mathcal{C}$ is said to be upper-triangular if there is no sequence

$$
x_{0} \stackrel{f_{0}}{\longrightarrow} x_{1} \stackrel{f_{1}}{\longrightarrow} \ldots \stackrel{f_{n-1}}{\longrightarrow} x_{n} \stackrel{f_{n}}{\longrightarrow} x_{0} \quad(n \geq 1)
$$

of nonzero morphisms in $\mathcal{C}$ unless $x_{0}=x_{1}=\cdots=x_{n}$. Alternatively, a linear category $\mathcal{C}$ is uppertriangular if there is a partial order $\leq$ on $\mathrm{Ob}(\mathcal{C})$ such that $\mathcal{C}(x, y) \neq 0$ implies $x \leq y$.

For a linear category $\mathcal{C}$, define a $\operatorname{ring} \operatorname{End}(\mathcal{C})$ by

$$
\operatorname{End}(\mathcal{C})=\bigoplus_{x \in \operatorname{Ob}(\mathcal{C})} \mathcal{C}(x, x)
$$

Lemma 3.6 ([8]). For an upper-triangular linear category $\mathcal{C}$, we have

$$
\mathrm{HH}_{*}(\mathcal{C}) \cong \bigoplus_{x \in \mathrm{Ob}(\mathcal{C})} \mathrm{HH}_{*}(\mathcal{C}(x, x)) \cong \mathrm{HH}_{*}(\operatorname{End}(\mathcal{C})) .
$$

Remark 14. Note that the ring $\operatorname{End}(\mathrm{C})$ is not unital if $\mathrm{Ob}(\mathrm{C})$ is infinite. The definition of the Hochschild homology of a non-unital ring $\mathrm{R}$ is different from the case of unital rings (see [41), but it is well known that the Hochschild homology of the direct sum of unital rings is isomorphic to the direct sum of the Hochschild homology of its direct summands. 
Definition 3.7. A strongly upper-triangular linear category is an upper-triangular linear category $\mathcal{C}$ such that for all $x \in \mathrm{Ob}(\mathcal{C})$, we have $\mathcal{C}(x, x) \cong \mathbb{Z}$.

Using that $\mathrm{HH}_{0}(\mathbb{Z})=\mathbb{Z}$ and for $n>0, \mathrm{HH}_{n}(\mathbb{Z})=0$, from the previous lemma we immediately get the following.

Corollary $3.8([8])$. For a strongly upper-triangular linear category $\mathcal{C}$, we have

$$
\begin{gathered}
\operatorname{Tr} \mathcal{C}=\operatorname{HH}_{0}(\mathcal{C}) \cong \operatorname{End}(\mathcal{C}) \cong \mathbb{Z} \mathrm{Ob}(\mathcal{C}), \\
\operatorname{HH}_{i}(\mathcal{C})=0 \quad \text { for } i>0 .
\end{gathered}
$$

Here $\mathbb{Z} \mathrm{Ob}(\mathcal{C})$ denotes the free $\mathbb{Z}$-module spanned by $\mathrm{Ob}(\mathcal{C})$.

Let $\mathcal{C}$ be an additive category, and let $B \subset \mathrm{Ob}(\mathcal{C})$ be a subset. Denote by $\left.\mathcal{C}\right|_{B}$ the full subcategory of $\mathcal{C}$ with $\mathrm{Ob}\left(\left.\mathcal{C}\right|_{B}\right)=B$.

The set $B$ is called a strongly upper-triangular basis of $\mathcal{C}$ if the following two conditions hold.

(1) The inclusion functor $\left.\mathcal{C}\right|_{B} \rightarrow \mathcal{C}$ induces equivalence of additive categories $\left(\left.\mathcal{C}\right|_{B}\right)^{\oplus} \simeq \mathcal{C}$.

(2) $\left.\mathcal{C}\right|_{B}$ is strongly upper-triangular.

Proposition $3.9([8])$. Let $\mathcal{C}$ be an additive category with a strongly upper-triangular basis $B$. Then we have

$$
\begin{gathered}
\operatorname{Tr} \mathcal{C}=\mathrm{HH}_{0}(\mathcal{C}) \cong \mathbb{Z} B \\
\mathrm{HH}_{i}(\mathcal{C})=0 \quad \text { for } i>0
\end{gathered}
$$

Remark 15 . For $\mathbb{k}$-linear categories it is natural to modify the Definition 3.7 to define a strongly uppertriangular $\mathbb{k}$-linear category by requiring that for all $x \in \mathrm{Ob}(\mathcal{C})$, we have $\mathcal{C}(x, x) \cong \mathbb{k}$. In that case, Corollary 3.8 and Proposition 3.9 remain valid with $\mathbb{Z}$ replaced by the field $\mathbb{k}$.

An additive category is said to be Krull-Schmidt if any object has a unique decomposition into a finite direct sum of objects having local endomorphism rings. That is to say, given an isomorphism $x_{1} \oplus x_{2} \oplus \cdots \oplus x_{r} \cong y_{1} \oplus y_{2} \oplus \cdots \oplus y_{s}$ where $x_{i}$ and $y_{j}$ are indecomposable, then $r=s$, and there exists some permutation $\sigma \in S_{r}$ such that $x_{\sigma(i)} \simeq y_{i}$.

Exercise 16. Show that any category $\mathcal{C}$ enriched in $\mathbf{V e c t}_{\mathbb{k}}$, i.e. the hom spaces form vector spaces and composition is a bilinear map, is Krull-Schmidt if and only if all idempotents split.

In a Krull-Schmidt category $\mathcal{C}$ the classes of indecomposables in the split Grothendieck group $K_{0}(\mathcal{C})$ give rise to a distinguished basis. When $\mathcal{C}$ is a graded linear category, then the graded hom between a pair of objects $x, y \in \mathrm{Ob}(\mathcal{C})$ is defined as

$$
\operatorname{HOM}_{\mathcal{C}}(x, y):=\bigoplus_{k \in \mathbb{Z}} \operatorname{Hom}_{\mathcal{C}}(x, y\langle k\rangle)
$$

When $\mathcal{C}$ is a graded $\mathbb{k}$-linear category, this graded hom gives rise to a sesquilinear inner product $\langle-,-\rangle: K_{0}(\mathcal{C}) \times K_{0}(\mathcal{C}) \rightarrow \mathbb{Z}((q))$ defined by

$$
\left\langle[x] \cong,[y]_{\cong}\right\rangle:=\operatorname{dim}_{q} \operatorname{HOM}_{\mathcal{C}}(x, y)=\sum_{k \in \mathbb{Z}} q^{k} \operatorname{dim} \operatorname{Hom}_{\mathcal{C}}(x, y\langle k\rangle) .
$$

One consequence of a graded linear category possessing a strongly upper triangular basis $B$ is that the classes of $B$ in the Grothendieck group satisfy the property that for any pair $x, y \in B$, we have

$$
\langle x, y\rangle \in \delta_{x, y}+q \mathbb{N}[q]
$$


in which case the basis resulting from $B$ is said to be almost orthogonal.

Remarks for experts. Strongly upper triangular bases often arise in geometric constructions where the categories of interest are categories of sheaves on certain varieties. Usually one starts with a triangulated category $\mathcal{C}$ and produces an abelian category $\mathcal{A}$ as the heart of a $t$-structure for $\mathcal{C}$. Distinguished basis $B$ arise as the simple objects in the heart of the corresponding $t$-structure. If $\mathcal{A}$ is endowed with a grading given by the cohomological Ext-grading from $\mathcal{C}$, then the resulting basis of simple objects is strongly upper triangular and their classes in the Grothendieck group are almost orthogonal.

For example, categories of perverse sheaves are the heart of a certain $t$-structure on the derived category of sheaves [6]. In this way, simple perverse sheaves naturally give rise to distinguished bases in geometric representation theory. The Kazhdan-Lusztig basis of a Hecke algebra associated to a Weyl group $W$ arise in this way 30 . Likewise, simple integrable representations and their tensor products of quantum Kac-Moody algebra possess canonical bases 42, 43, 44, 45, 27, 28. Outside of geometry, there is a $t$-structure on the bounded derived category $D^{b}(\Lambda-\bmod )$ of a finite-dimensional $\mathbb{k}$-algebra $\Lambda$ in which the simple $\Lambda$-modules form a strongly upper triangular basis in the heart of the $t$-structure [1].

\section{Categorified QuANtum Groups}

Fix a base field $\mathbb{k}$. We will always work over this field which is not assumed to be of characteristic 0 , nor algebraically closed.

\subsection{Quantum $\mathfrak{s l}_{n}$.}

4.1.1. The Cartan datum. Let $I=\{1,2, \ldots, n-1\}$ consist of the set of vertices of the Dynkin diagram of type $A_{n-1}$

$$
\stackrel{1}{2}-3
$$

enumerated from left to right. Let $X=\mathbb{Z}^{n-1}$ denote the weight lattice for $\mathfrak{s l}_{n}$ and $\left\{\alpha_{i}\right\}_{i \in I} \subset X$ and $\left\{\Lambda_{i}\right\}_{i \in I} \subset X$ denote the collection of simple roots and fundamental weights, respectively. There is a symmetric bilinear form on $X$ defined by $\left(\alpha_{i}, \alpha_{j}\right)=a_{i j}$ where

$$
a_{i j}= \begin{cases}2 & \text { if } i=j \\ -1 & \text { if }|i-j|=1 \\ 0 & \text { if }|i-j|>1\end{cases}
$$

is the (symmetric) Cartan matrix associated to $\mathfrak{s l}_{n}$. For $i \in I$ denote the simple coroots by $h_{i} \in X^{\vee}=$ $\operatorname{Hom}_{\mathbb{Z}}(X, \mathbb{Z})$. Write $\langle\cdot, \cdot\rangle: X^{\vee} \times X \rightarrow \mathbb{Z}$ for the canonical pairing $\langle i, \lambda\rangle:=\left\langle h_{i}, \lambda\right\rangle=2 \frac{\left(\alpha_{i}, \lambda\right)}{\left(\alpha_{i}, \alpha_{i}\right)}$ for $i \in I$ and $\lambda \in X$ that satisfies $\left\langle h_{i}, \Lambda_{i}\right\rangle=\delta_{i, j}$. Any weight $\lambda \in X$ can be written as $\lambda=\left(\lambda_{1}, \lambda_{2}, \ldots, \lambda_{n-1}\right)$, where $\lambda_{i}=\left\langle h_{i}, \lambda\right\rangle$.

We let $X^{+} \subset X$ denote the dominant weights, which are those of the form $\sum_{i} \lambda_{i} \Lambda_{i}$ with $\lambda_{i} \geq 0$. Finally, let $[k]=\frac{q^{k}-q^{-k}}{q-q^{-1}}$ and $[k] !=[k][k-1] \ldots[1]$.

4.1.2. The algebra $\mathbf{U}_{q}\left(\mathfrak{s l}_{n}\right)$. The algebra $\mathbf{U}_{q}\left(\mathfrak{s l}_{n}\right)$ is the $\mathbb{Q}(q)$-algebra with unit generated by the elements $E_{i}, F_{i}$ and $K_{i}^{ \pm 1}$ for $i=1,2, \ldots, n-1$, with the defining relations

$$
\begin{gathered}
K_{i} K_{i}^{-1}=K_{i}^{-1} K_{i}=1, \quad K_{i} K_{j}=K_{j} K_{i}, \\
K_{i} E_{j} K_{i}^{-1}=q^{a_{i j}} E_{j}, \quad K_{i} F_{j} K_{i}^{-1}=q^{-a_{i j}} F_{j}, \\
E_{i} F_{j}-F_{j} E_{i}=\delta_{i j} \frac{K_{i}-K_{i}^{-1}}{q-q^{-1}}, \\
E_{i}^{2} E_{j}-\left(q+q^{-1}\right) E_{i} E_{j} E_{i}+E_{j} E_{i}^{2}=0 \text { if } j=i \pm 1,
\end{gathered}
$$




$$
\begin{gathered}
F_{i}^{2} F_{j}-\left(q+q^{-1}\right) F_{i} F_{j} F_{i}+F_{j} F_{i}^{2}=0 \text { if } j=i \pm 1, \\
E_{i} E_{j}=E_{j} E_{i}, \quad F_{i} F_{j}=F_{j} F_{i} \text { if }|i-j|>1 .
\end{gathered}
$$

Let $\dot{\mathbf{U}}\left(\mathfrak{s l}_{n}\right)$ be the idempotented version of $\mathbf{U}_{q}\left(\mathfrak{s l}_{n}\right)$ where the unit is replaced by a collection of orthogonal idempotents $1_{\lambda}$ indexed by the weight lattice $X$ of $\mathfrak{s l}_{n}$,

$$
1_{\lambda} 1_{\lambda^{\prime}}=\delta_{\lambda \lambda^{\prime}} 1_{\lambda},
$$

such that if $\lambda=\left(\lambda_{1}, \lambda_{2}, \ldots, \lambda_{m-1}\right)$, then

$$
K_{i} 1_{\lambda}=1_{\lambda} K_{i}=q^{\lambda_{i}} 1_{\lambda}, \quad E_{i} 1_{\lambda}=1_{\lambda+\alpha_{i}} E_{i}, \quad F_{i} 1_{\lambda}=1_{\lambda-\alpha_{i}} F_{i},
$$

where

$$
\lambda+\alpha_{i}=\left\{\begin{array}{cl}
\left(\lambda_{1}+2, \lambda_{2}-1, \lambda_{3}, \ldots, \lambda_{m-2}, \lambda_{m-1}\right) & \text { if } i=1 \\
\left(\lambda_{1}, \lambda_{2}, \ldots, \lambda_{m-2}, \lambda_{m-1}-1, \lambda_{m-1}+2\right) & \text { if } i=n-1 \\
\left(\lambda_{1}, \ldots, \lambda_{i-1}-1, \lambda_{i}+2, \lambda_{i+1}-1, \ldots, \lambda_{m-1}\right) & \text { otherwise. }
\end{array}\right.
$$

$\dot{\mathbf{U}}\left(\mathfrak{s l}_{n}\right)$ can be viewed as a category with objects $\lambda \in X$ and morphisms given by compositions of $E_{i}$ and $F_{i}$ with $1 \leq i<n$ modulo the above relations.

Let $\mathcal{A}:=\mathbb{Z}\left[q, q^{-1}\right] ;$ the $\mathcal{A}$-algebra ${ }_{\mathcal{A}} \dot{\mathbf{U}}\left(\mathfrak{s l} l_{n}\right)$ is the integral form of $\dot{\mathbf{U}}\left(\mathfrak{s l}_{n}\right)$ generated by products of divided powers $E_{i}^{(a)} 1_{\lambda}:=\frac{E_{i}^{a}}{[a] !} 1_{\lambda}, F_{i}^{(a)} 1_{\lambda}:=\frac{F_{i}^{a}}{[a] !} 1_{\lambda}$ for $\lambda \in X$ and $i=1,2, \ldots, n-1$.

4.2. The 2-category $\mathcal{U}_{Q}\left(\mathfrak{s l}_{n}\right)$. Here we describe a categorification of $\mathbf{U}\left(\mathfrak{s l}_{n}\right)$ mainly following [19] and 33. For an elementary introduction to the categorification of $\mathfrak{s l}_{2}$ see 38 .

4.2.1. Choice of scalars $Q$. Associated to the Cartan datum for $\mathfrak{s l}_{n}$ we also fix a choice of scalars $Q$ consisting of:

- $t_{i j}$ for all $i, j \in I$,

such that

- $t_{i i}=1$ for all $i \in I$ and $t_{i j} \in \mathbb{k}^{\times}$for $i \neq j$,

- $t_{i j}=t_{j i}$ when $a_{i j}=0$.

4.2.2. The definition. By a graded linear 2-category we mean a category enriched in graded linear categories, so that the hom spaces form graded linear categories, and the composition map is grading preserving.

Given a fixed choice of scalars $Q$ we can define the following 2-category.

Definition 4.1. The 2-category $\mathcal{U}_{Q}\left(\mathfrak{s l}_{n}\right)$ is the graded linear 2-category consisting of:

- objects $\lambda$ for $\lambda \in X$.

- 1-morphisms are formal direct sums of (shifts of) compositions of

$$
\mathbf{1}_{\lambda}, \quad \mathbf{1}_{\lambda+\alpha_{i}} \mathcal{E}_{i}=\mathbf{1}_{\lambda+\alpha_{i}} \mathcal{E}_{i} \mathbf{1}_{\lambda}=\mathcal{E}_{i} \mathbf{1}_{\lambda}, \quad \text { and } \quad \mathbf{1}_{\lambda-\alpha_{i}} \mathcal{F}_{i}=\mathbf{1}_{\lambda-\alpha_{i}} \mathcal{F}_{i} \mathbf{1}_{\lambda}=\mathcal{F}_{i} \mathbf{1}_{\lambda}
$$

for $i \in I$ and $\lambda \in X$.

- 2-morphisms are $\mathbb{k}$-vector spaces spanned by compositions of (decorated) tangle-like diagrams illustrated below.

$$
\begin{array}{cc}
\left.{ }_{i}^{\lambda+\alpha_{i}}\right\}^{\lambda}: \mathcal{E}_{i} \mathbf{1}_{\lambda} \rightarrow \mathcal{E}_{i} \mathbf{1}_{\lambda}\left\langle\left(\alpha_{i}, \alpha_{i}\right)\right\rangle & \left.{ }^{\lambda-\alpha_{i}}\right\}_{i}^{\lambda}: \mathcal{F}_{i} \mathbf{1}_{\lambda} \rightarrow \mathcal{F}_{i} \mathbf{1}_{\lambda}\left\langle\left(\alpha_{i}, \alpha_{i}\right)\right\rangle \\
{ }_{i}{ }_{\lambda}: \mathcal{E}_{i} \mathcal{E}_{j} \mathbf{1}_{\lambda} \rightarrow \mathcal{E}_{j} \mathcal{E}_{i} \mathbf{1}_{\lambda}\left\langle-\left(\alpha_{i}, \alpha_{j}\right)\right\rangle & \chi_{i}{ }^{\lambda}: \mathcal{F}_{i} \mathcal{F}_{j} \mathbf{1}_{\lambda} \rightarrow \mathcal{F}_{j} \mathcal{F}_{i} \mathbf{1}_{\lambda}\left\langle-\left(\alpha_{i}, \alpha_{j}\right)\right\rangle
\end{array}
$$




$$
\begin{array}{ll}
\mathcal{\gamma}_{\lambda}^{i}: \mathbf{1}_{\lambda} \rightarrow \mathcal{F}_{i} \mathcal{E}_{i} \mathbf{1}_{\lambda}\left\langle 1+\left(\lambda, \alpha_{i}\right)\right\rangle & y^{i}{ }_{\lambda}: \mathbf{1}_{\lambda} \rightarrow \mathcal{E}_{i} \mathcal{F}_{i} \mathbf{1}_{\lambda}\left\langle 1-\left(\lambda, \alpha_{i}\right)\right\rangle \\
\overbrace{}^{\lambda}: \mathcal{F}_{i} \mathcal{E}_{i} \mathbf{1}_{\lambda} \rightarrow \mathbf{1}_{\lambda}\left\langle 1+\left(\lambda, \alpha_{i}\right)\right\rangle & \overbrace{}^{i}: \mathcal{E}_{i} \mathcal{F}_{i} \mathbf{1}_{\lambda} \rightarrow \mathbf{1}_{\lambda}\left\langle 1-\left(\lambda, \alpha_{i}\right)\right\rangle
\end{array}
$$

Here we follow the grading conventions in [19] and [40] which are opposite to those from [33]. In this 2-category (and those throughout the paper) we read diagrams from right to left and bottom to top. The identity 2 -morphism of the 1-morphism $\mathcal{E}_{i} \mathbf{1}_{\lambda}$ is represented by an upward oriented line labeled by $i$ and the identity 2 -morphism of $\mathcal{F}_{i} \mathbf{1}_{\lambda}$ is represented by a downward such line.

The 2-morphisms satisfy the following relations:

(1) The 1-morphisms $\mathcal{E}_{i} \mathbf{1}_{\lambda}$ and $\mathcal{F}_{i} \mathbf{1}_{\lambda}$ are biadjoint (up to a specified degree shift). These conditions are expressed diagrammatically as
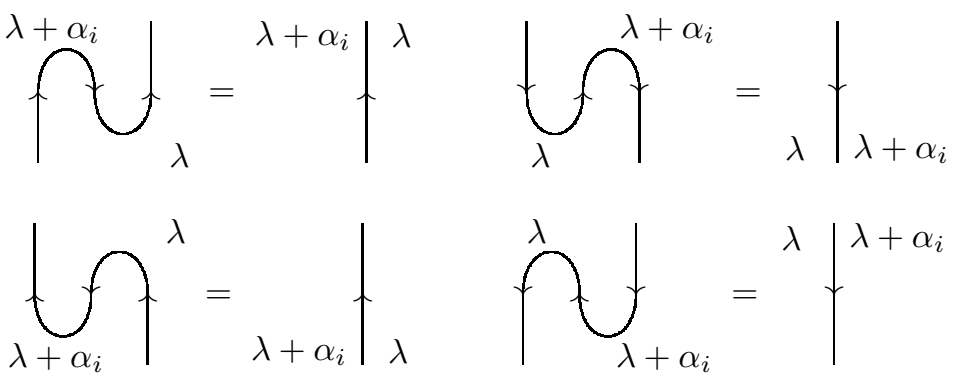

(2) The 2-morphisms are $Q$-cyclic with respect to this biadjoint structure.

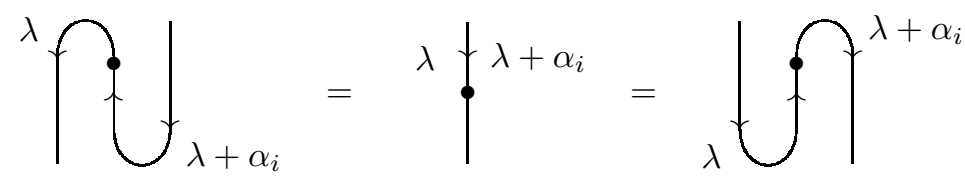

The $Q$-cyclic relations for crossings are given by

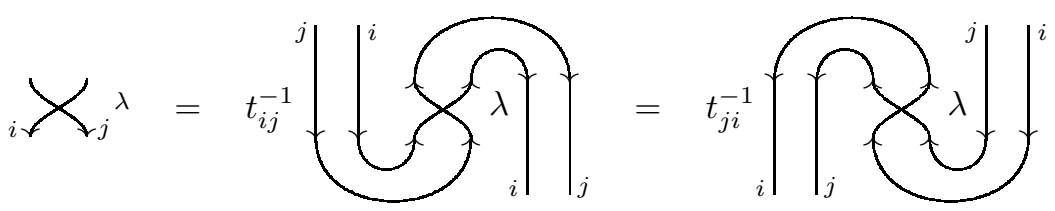

Sideways crossings can then be defined utilizing the $Q$-cyclic condition by the equalities:

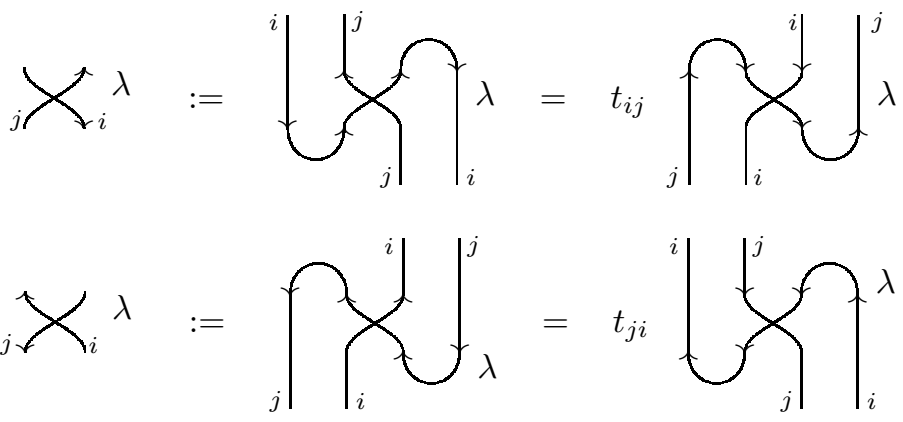

where the second equality in (4.14) and (4.15) follow from (4.13). 
(3) The $\mathcal{E}$ 's carry an action of the KLR algebra associated to $Q$. The KLR algebra $R=R_{Q}$ associated to $Q$ is defined by finite $\mathbb{k}$-linear combinations of braid-like diagrams in the plane, where each strand is labeled by a vertex $i \in I$. Strands can intersect and can carry dots but triple intersections are not allowed. Diagrams are considered up to planar isotopy that do not change the combinatorial type of the diagram. We recall the local relations:

i) If all strands are labeled by the same $i \in I$ then the nilHecke algebra axioms (2.3) and (2.4) hold.

ii) For $i \neq j$

$$
\bigotimes_{i}^{\lambda}= \begin{cases}t_{i j}{ }_{i} \uparrow \uparrow_{j} & \text { if }\left(\alpha_{i}, \alpha_{j}\right)=0, \\ t_{i j} \uparrow_{i} \uparrow \uparrow_{j}+t_{j i} \uparrow_{i} & \text { if }\left(\alpha_{i}, \alpha_{j}\right) \neq 0,\end{cases}
$$

iii) For $i \neq j$ the dot sliding relations

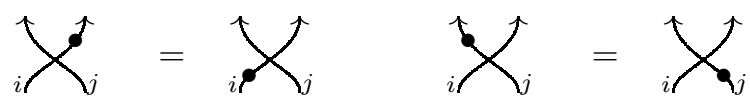

hold.

iv) Unless $i=k$ and $\left(\alpha_{i}, \alpha_{j}\right)<0$ the relation

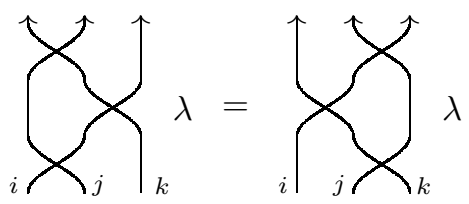

holds. Otherwise, $\left(\alpha_{i}, \alpha_{j}\right)=-1$ and

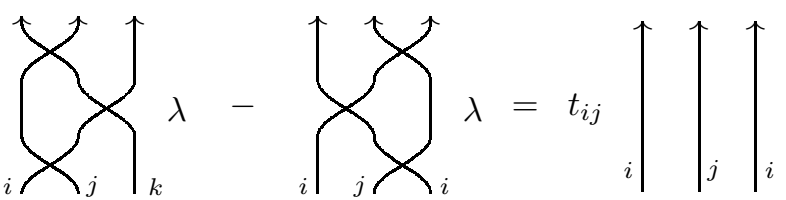

(4) When $i \neq j$ one has the mixed relations relating $\mathcal{E}_{i} \mathcal{F}_{j}$ and $\mathcal{F}_{j} \mathcal{E}_{i}$ :

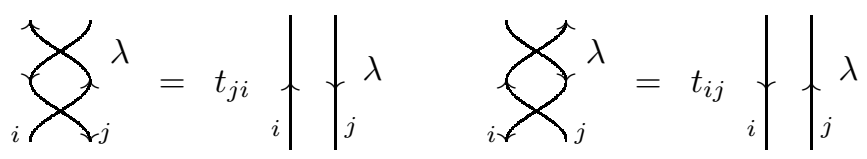

(5) Negative degree bubbles are zero. That is, for all $m \in \mathbb{Z}_{+}$one has

$$
\bigcap_{m}^{\lambda}=0 \text { if } m<\lambda_{i}-1, \quad \overbrace{m}^{\lambda}=0 \text { if } m<-\lambda_{i}-1 \text {. }
$$

On the other hand, a dotted bubble of degree zero is just the identity 2-morphism:

$$
\bigcap_{\lambda_{i}-1}^{\lambda}=\operatorname{Id}_{\mathbf{1}_{\lambda}} \text { for } \lambda_{i} \geq 1, \quad \bigodot_{-\lambda_{i}-1}^{\lambda}=\operatorname{Id}_{\mathbf{1}_{\lambda}} \text { for } \lambda_{i} \leq-1 \text {. }
$$


(6) For any $i \in I$ one has the extended $\mathfrak{s l}_{2}$-relations. In order to describe certain extended $\mathfrak{s l}_{2}$ relations it is convenient to use a shorthand notation from [37 called fake bubbles. These are diagrams for dotted bubbles where the labels of the number of dots is negative, but the total degree of the dotted bubble taken with these negative dots is still positive. They allow us to write these extended $\mathfrak{s l}_{2}$ relations more uniformly (i.e. independent on whether the weight $\lambda_{i}$ is positive or negative).

- Degree zero fake bubbles are equal to the identity 2-morphisms

$$
\bigodot_{\lambda_{i}-1}^{\lambda}=\operatorname{Id}_{\mathbf{1}_{\lambda}} \text { if } \lambda_{i} \leq 0, \quad \bigodot_{-\lambda_{i}-1}^{i}=\operatorname{Id}_{\mathbf{1}_{\lambda}} \text { if } \lambda_{i} \geq 0 \text {. }
$$

- Higher degree fake bubbles for $\lambda_{i}<0$ are defined inductively as

$$
\bigcap_{\lambda_{i}-1+j}^{\lambda}= \begin{cases}-\sum_{\substack{a+b=j \\ b \geq 1}} \bigcap_{\lambda_{i}-1+a}^{\lambda} \bigodot_{-\lambda-1+b} \text { if } 0 \leq j<-\lambda_{i}+1 \\ 0 & \text { if } j<0 .\end{cases}
$$

- Higher degree fake bubbles for $\lambda_{i}>0$ are defined inductively as

$$
\overbrace{-\lambda_{i}-1+j}^{\lambda}= \begin{cases}-\sum_{\substack{a+b=j \\ a \geq 1}} \bigodot_{\lambda_{i}-1+a}^{\lambda} \bigodot_{-\lambda-1+b} \text { if } 0 \leq j<\lambda_{i}+1 \\ 0 & \text { if } j<0 .\end{cases}
$$

These equations arise from the homogeneous terms in $t$ of the 'infinite Grassmannian' equation

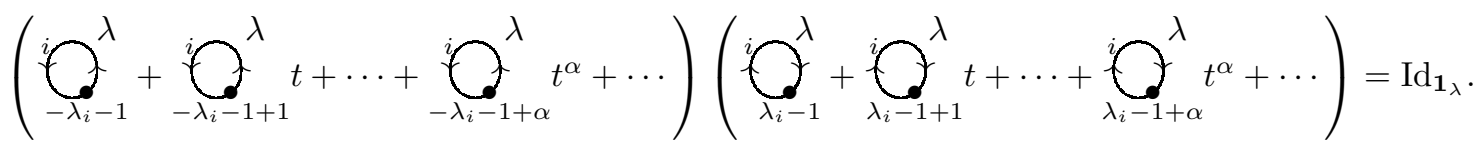

Now we can define the extended $\mathfrak{s l}_{2}$ relations. Note that in [19] additional curl relations were provided that can be derived from those above. Here we provide a minimal set of relations.

If $\lambda_{i}>0$ then we have:

$$
\begin{aligned}
& \chi_{i} \bigcap^{\lambda}=0{ }_{i}^{\lambda}=-\bigotimes_{i}^{\lambda} \\
& \left.f_{i}\right|_{i} ^{\lambda}=-\bigodot_{i}^{\lambda}+\sum_{\substack{f_{1}+f_{2}+f_{3} \\
=\lambda_{i}-1}}^{\overbrace{-\lambda_{i}-1+f_{2}}^{f_{3}}}
\end{aligned}
$$

If $\lambda_{i}<0$ then we have:

$$
\left.\left.\mathcal{X}_{\uparrow_{i}}^{\lambda}\right|_{i}\right|_{i} ^{\lambda}=-\bigotimes_{i}^{\lambda}
$$




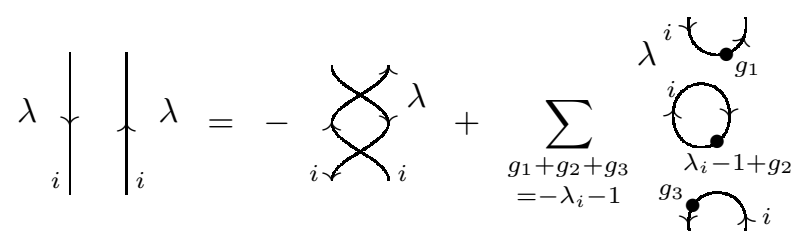

If $\lambda_{i}=0$ then we have:

$$
\begin{aligned}
& \chi_{i}^{\lambda}={ }^{\lambda}{ }_{i}^{\lambda} \hat{X}_{\gamma_{i}}^{\lambda}={ }^{\lambda} \uparrow \\
& \left.\uparrow_{i}\right|_{i} ^{\lambda}=-\left.\left.\sum_{i}^{l}\right|_{i}\right|_{i} ^{\lambda}=-\sum_{i}^{\lambda}
\end{aligned}
$$

While the definition above may seem quite involved, the relations above encode a great deal of interesting combinatorics. In particular, the relations above imply that all of the equations between generators in $\dot{\mathbf{U}}\left(\mathfrak{s l}_{n}\right)$ lift to explicit isomorphisms in $\mathcal{U}_{Q}\left(\mathfrak{s l}_{n}\right)$. For more details see [33].

In what follows it is often convenient to introduce a shorthand notation
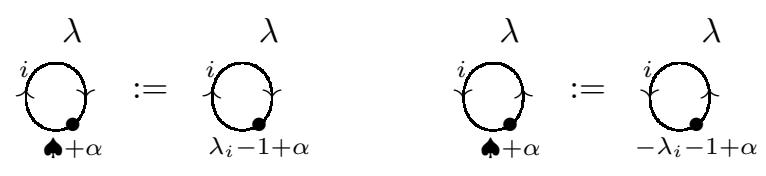

for all $\lambda_{i}$. Note that as long as $\alpha \geq 0$ this notation makes sense even when $+\alpha<0$. These negative values are the fake bubbles defined above.

Exercise 17. Use the relations above to show that the following bubble slide equations

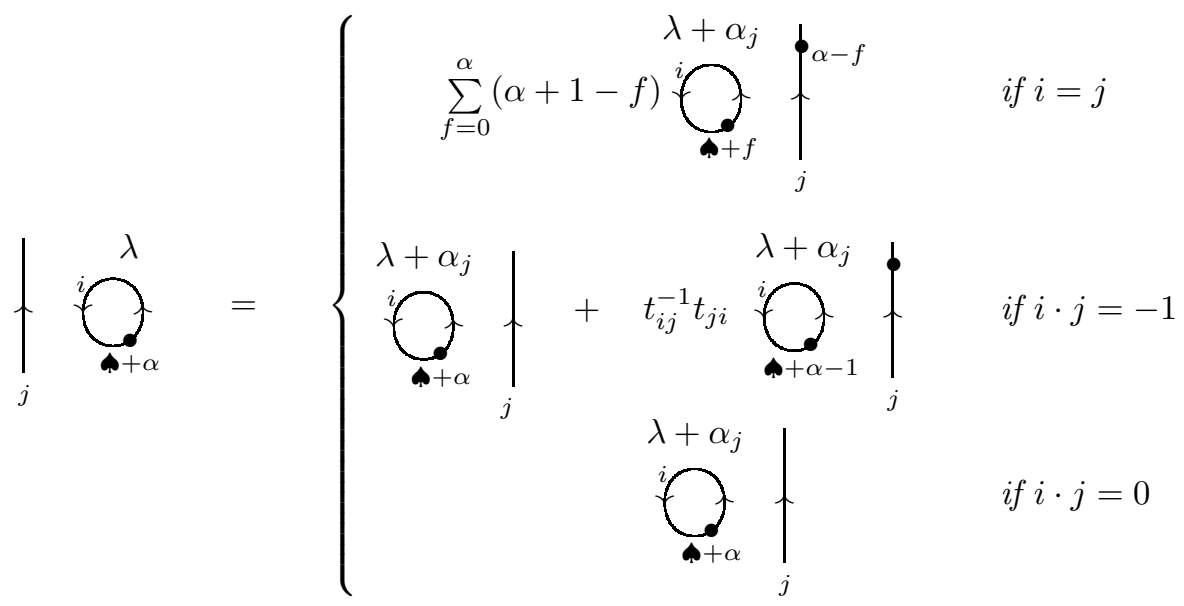



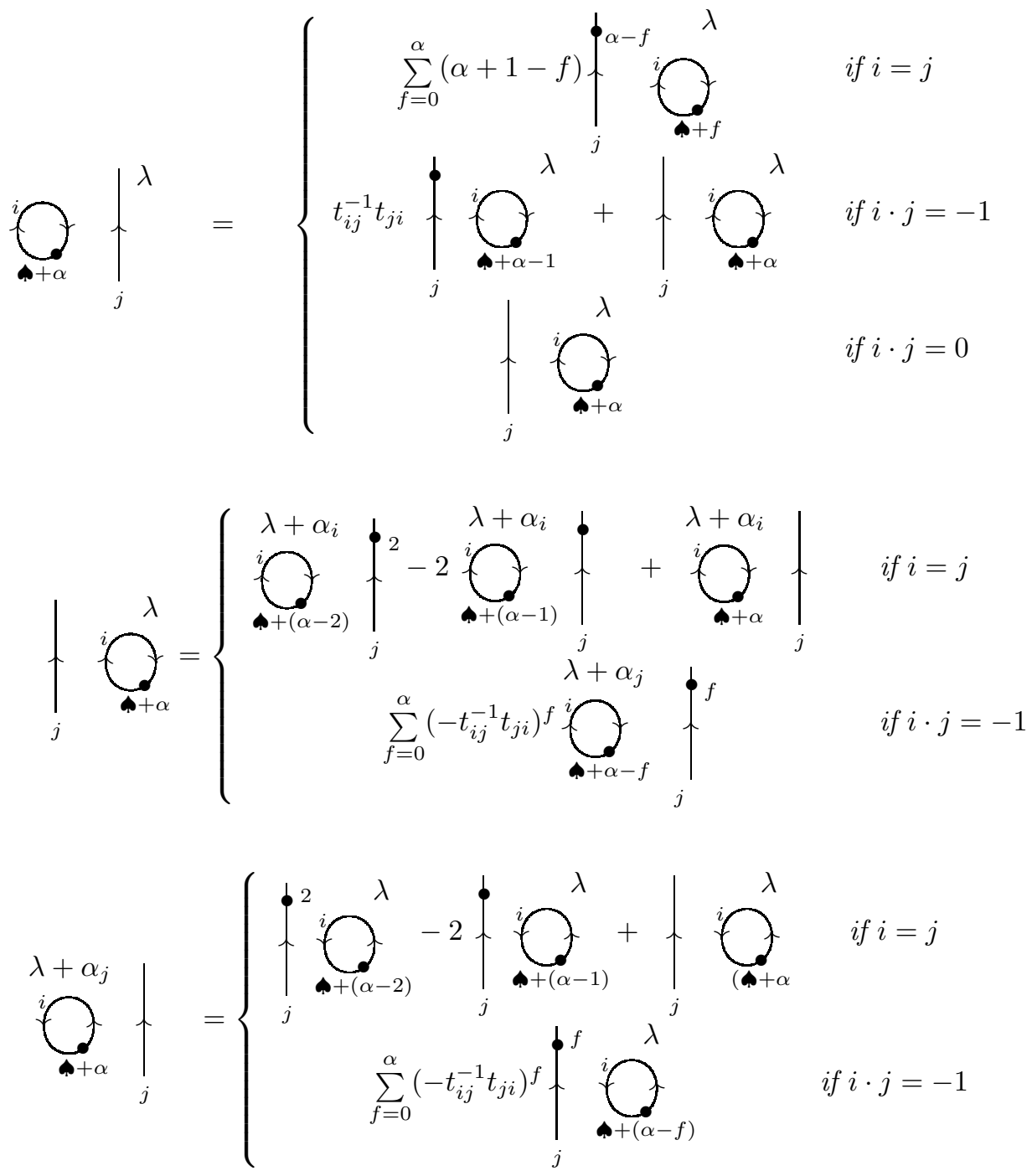

hold in $\mathcal{U}_{Q}\left(\mathfrak{s l}_{n}\right)$. Hint: see equations (6.8) and (6.9) of [19].

4.3. Symmetric functions and bubbles. The calculus of closed diagrams in the 2-category $\mathcal{U}_{Q}\left(\mathfrak{s l}_{n}\right)$ is remarkably rich. A prominent role is played by the non-nested dotted bubbles of a fixed orientation since any closed diagram in the graphical calculus for $\mathcal{U}_{Q}\left(\mathfrak{s l}_{n}\right)$ can be reduced to composites of such diagrams.

There is a beautiful analogy between dotted bubbles in the graphical calculus and various bases for the ring of symmetric functions. In [37] it is shown that there is an isomorphism

$$
\begin{aligned}
\psi_{\lambda}: \operatorname{Sym} & \longrightarrow Z(\lambda)=\mathcal{U}_{Q}\left(\mathfrak{s l}_{2}\right)\left(\mathbf{1}_{\lambda}, \mathbf{1}_{\lambda}\right) \\
h_{r} & \mapsto \underbrace{\lambda}_{\mathfrak{\uparrow}+r}
\end{aligned}
$$




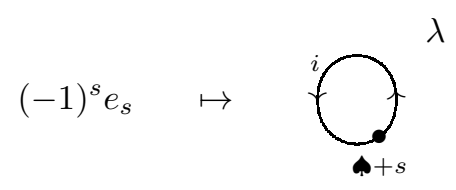

$\lambda$

where Sym denotes the ring of symmetric functions, $h_{r}$ denotes the complete symmetric function of degree $r$, and $e_{s}$ denotes the elementary symmetric function of degree $s$. In fact, under this isomorphisms the well known relationship between complete and elementary symmetric functions becomes the infinite Grassmannian equation (4.24).

It is well known that for a partition $\lambda=\left(\lambda_{1}, \ldots, \lambda_{n}\right)$ with $\lambda_{1} \geq \lambda_{2} \geq \cdots \geq \lambda_{n}$, products of elementary symmetric functions $e_{\lambda}=e_{\lambda_{1}} \ldots e_{\lambda_{n}}$ form a basis for Sym, see for example [46]. Likewise, products of complete symmetric functions also provide a basis for Sym. This mirrors the fact that any closed diagram in the graphical calculus for $\mathcal{U}_{Q}\left(\mathfrak{s l}_{2}\right)$ can be reduced to a product on non-nested bubbles of a given orientation.

In the $\mathfrak{s l}_{n}$ calculus of the 2-category $\mathcal{U}\left(\mathfrak{s l}_{n}\right)$, we have the isomorphism

$$
\psi_{\lambda}: \prod_{i \in I} \operatorname{Sym} \longrightarrow Z(\lambda)=\mathcal{U}_{Q}\left(\mathfrak{s l}_{n}\right)\left(\mathbf{1}_{\lambda}, \mathbf{1}_{\lambda}\right)
$$

since any closed diagram can still be reduced to products of non-nested closed bubbles labelled by $i \in I$.

In what follows, it will be interesting to consider which products of closed diagrams correspond to the basis of Sym given by the power sum $p_{r}$ symmetric functions. Using a formula that expresses power sum symmetric functions in terms of products of complete and elementary symmetric functions, we can denote by $p_{i, r}(\lambda)$ for $r>0$, the image of the power symmetric polynomial on $i$-labelled strands in

$$
\begin{aligned}
& \begin{array}{lll}
\lambda & \lambda & \lambda
\end{array}
\end{aligned}
$$

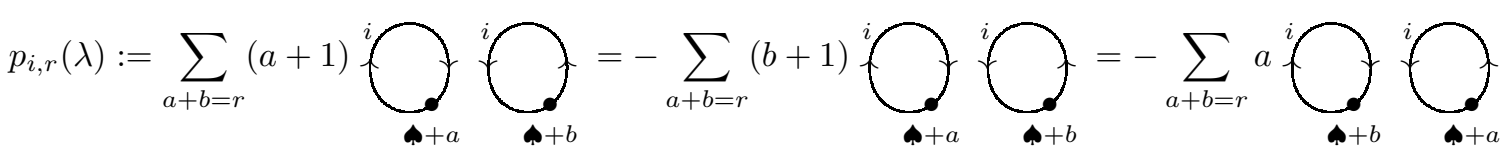

For later convenience we set $p_{i, 0}(\lambda)=\lambda_{i}$.

Exercise 18. Using the bubble slide formulas from exercise 17, show that

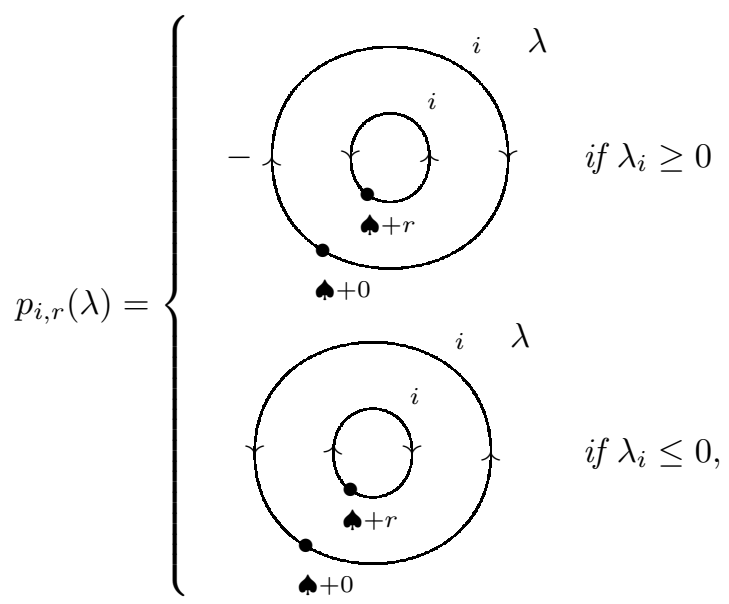

so that the formulas defining $p_{r}(\lambda)$ can be obtained by simplifying a degree $r$ bubble with one orientation nested inside of a degree zero bubble with the opposite orientation. 
Note that other interesting basis for symmetric functions also have natural graphical analogs. In 34] diagrams corresponding to the basis of Schur functions are given.

Exercise 19. Using the bubble sliding equations from exercise 17, prove the following power sum slide rules

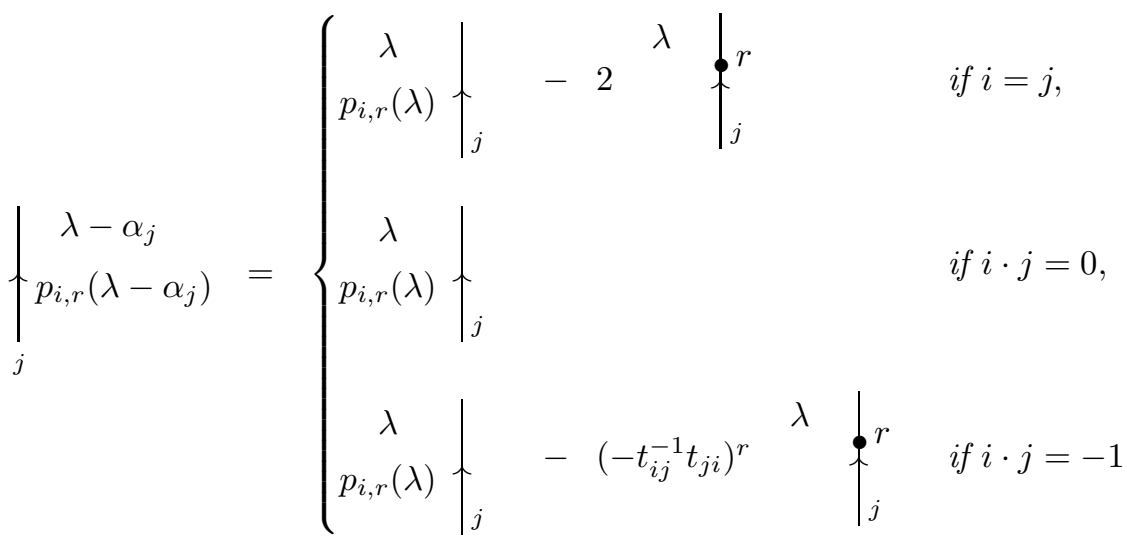

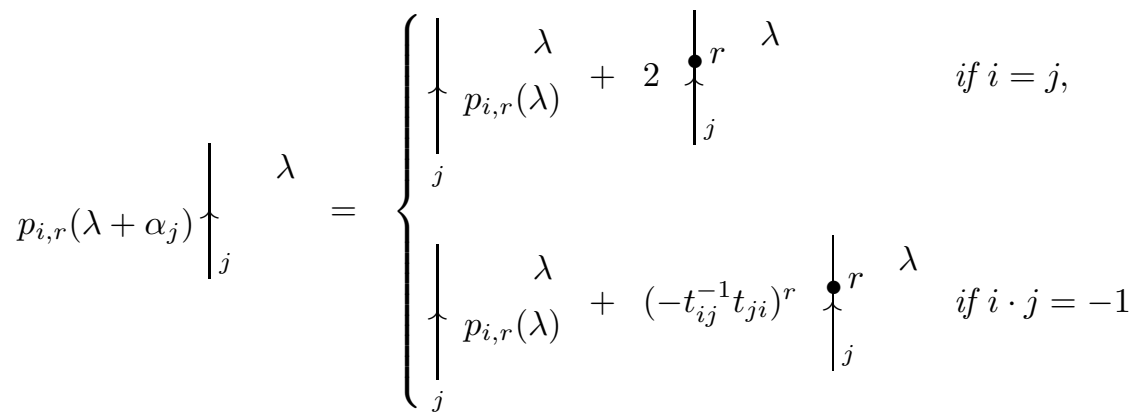

4.4. Kar, $K_{0}$ and $\operatorname{Tr}$ for 2-categories. We can extend many of the constructions from section 3 defined for (additive) linear categories to the 2-categorical setting. A 2-category $\mathbf{C}$ is linear if the categories $\mathbf{C}(x, y)$ are linear for all $x, y \in \mathrm{Ob}(\mathbf{C})$ and the composition functor preserves the linear structure (see [10] for more details). Similarly, an additive linear 2-category is a linear 2-category in which the categories $\mathbf{C}(x, y)$ are also additive and composition is given by an additive functor.

The following definitions extend the Karoubi envelope, split Grothendieck group, and trace to the 2-categorical setting.

- Given an additive linear 2-category $\mathbf{C}$, define the split Grothendieck group $K_{0}(\mathbf{C})$ of $\mathbf{C}$ to be the linear category with $\mathrm{Ob}\left(K_{0}(\mathbf{C})\right)=\mathrm{Ob}(\mathbf{C})$ and with $K_{0}(\mathbf{C})(x, y):=K_{0}(\mathbf{C}(x, y))$ for any two objects $x, y \in \mathrm{Ob}(\mathbf{C})$. For $[f] \cong \in \mathrm{Ob}\left(K_{0}(\mathbf{C})(x, y)\right)$ and $[g]_{\cong} \in \mathrm{Ob}\left(K_{0}(\mathbf{C})(y, z)\right)$ the composition in $K_{0}(\mathbf{C})$ is defined by $[g] \cong \circ[f] \cong:=[g \circ f] \cong$.

- The trace $\operatorname{Tr}(\mathbf{C})$ of a linear 2-category is the linear category with $\operatorname{Ob}(\operatorname{Tr}(\mathbf{C}))=\operatorname{Ob}(\mathbf{C})$ and with $\operatorname{Tr}(\mathbf{C})(x, y):=\operatorname{Tr}(\mathbf{C}(x, y))$ for any two objects $x, y \in \operatorname{Ob}(\mathbf{C})$. For $\sigma \in \operatorname{End}_{\mathbf{C}(x, y)}$ and $\tau \in \operatorname{End}_{\mathbf{C}(y, z)}$, we have $[\tau] \circ[\sigma]=[\tau \circ \sigma]$. The identity morphism for $x \in \operatorname{Ob}(\operatorname{Tr}(\mathbf{C}))=\operatorname{Ob}(\mathbf{C})$ is given by $\left[1_{I_{x}}\right]$.

- The Karoubi envelope $\operatorname{Kar}(\mathbf{C})$ of a linear 2-category $\mathbf{C}$ is the linear 2-category with $\operatorname{Ob}(\operatorname{Kar}(\mathbf{C}))=$ $\mathrm{Ob}(\mathbf{C})$ and with hom categories $\operatorname{Kar}(\mathbf{C})(x, y):=\operatorname{Kar}(\mathbf{C}(x, y))$. The composition functor $\operatorname{Kar}(\mathbf{C})(y, z) \times \operatorname{Kar}(\mathbf{C})(x, y) \rightarrow \operatorname{Kar}(\mathbf{C})(x, z)$ is induced by the universal property of the Karoubi envelope from the composition functor in $\mathbf{C}$. The fully-faithful additive functors $\mathbf{C}(x, y) \rightarrow \operatorname{Kar}(\mathbf{C}(x, y))$ combine to form an additive 2-functor $\mathbf{C} \rightarrow \operatorname{Kar}(\mathbf{C})$ that is universal with respect to splitting idempotents in the Hom-categories $\mathbf{C}(x, y)$. 
For categorification using $K_{0}$ it is necessary to consider the 2-category $\operatorname{Kar}\left(\mathcal{U}\left(\mathfrak{s l}_{n}\right)\right)$. We will denote by $\dot{\mathcal{U}}$ the Karoubi envelope of $\mathcal{U}$.

The homomorphisms $h_{\mathbf{C}(x, y)}$ taken over all objects $x, y \in \mathrm{Ob}(\mathbf{C})$ give rise to a linear functor

$$
h_{\mathbf{C}}: \mathcal{K}_{0}(\mathbf{C}) \rightarrow \operatorname{Tr}(\mathbf{C})
$$

which is the identity map on objects and sends $K_{0}(\mathbf{C})(x, y) \rightarrow \operatorname{Tr}(\mathbf{C})(x, y)$ via the homomorphism $h_{\mathbf{C}(x, y)}$. It is easy to see that this assignment preserves composition since

$$
h_{\mathbf{C}}([g] \cong \circ[f] \cong)=h_{\mathbf{C}}([g \circ f] \cong)=\left[1_{g \circ f}\right]=\left[1_{g} \circ 1_{f}\right]=\left[1_{g}\right] \circ\left[1_{f}\right]=h_{\mathbf{C}}([g] \cong) \circ h_{\mathbf{C}}([f] \cong) .
$$

In the following sections we study the trace and $K_{0}$ for the 2 -categories $\dot{\mathcal{U}}\left(\mathfrak{s l}_{n}\right)$.

4.5. Two categorifications of Lusztig's integral form ${ }_{\mathcal{A}} \dot{\mathbf{U}}\left(\mathfrak{s l}_{2}\right)$. Here we present the results of [37, 34] computing $K_{0}$ and of [8] computing $\operatorname{Tr}$ of $\mathcal{U}\left(\mathfrak{s l}_{2}\right)$.

The algebra ${ }_{\mathcal{A}} \dot{\mathbf{U}}\left(\mathfrak{s l}_{2}\right)$ has a well-known basis, called Lusztig's canonical basis $\mathbb{B}$ consisting of

(i) $E^{(a)} F^{(b)} 1_{n} \quad$ for $a, b \geq 0, n \in \mathbb{Z}, n \leq b-a$,

(ii) $F^{(b)} E^{(a)} 1_{n} \quad$ for $a, b \geq 0, n \in \mathbb{Z}, n \geq b-a$, where $E^{(a)} F^{(b)} 1_{b-a}=F^{(b)} E^{(a)} 1_{b-a}$. Let ${ }_{m} \mathbb{B}_{n}$ be set of elements in $\mathbb{B}$ belonging to $1_{m}\left({ }_{\mathcal{A}} \dot{\mathbf{U}}\right) 1_{n}$.

We associate to each $x \in \mathbb{B}$ a 1 -morphism in $\dot{\mathcal{U}}$ as follows:

$$
x \mapsto \mathcal{E}(x):= \begin{cases}\mathcal{E}^{(a)} \mathcal{F}^{(b)} \mathbf{1}_{n} & \text { if } x=E^{(a)} F^{(b)} 1_{n}, \\ \mathcal{F}^{(b)} \mathcal{E}^{(a)} \mathbf{1}_{n} & \text { if } x=F^{(b)} E^{(a)} 1_{n} .\end{cases}
$$

Let $\mathcal{B}=\{\mathcal{E}(x) \mid x \in \mathbb{B}\}$ and ${ }_{m} \mathcal{B}_{n}=\left\{\mathcal{E}(x) \mid x \in{ }_{m} \mathbb{B}_{n}\right\}$.

The first categorification of ${ }_{\mathcal{A}} \dot{\mathbf{U}}$ was given in [37] where the decategorification functor is given by $K_{0}$. The original proof worked with the 2-category $\dot{\mathcal{U}}\left(\mathfrak{s l}_{2}\right)$ defined over a field $\mathbb{k}$, so that spaces of 2-morphisms formed vector spaces and the Krull-Schmidt property could be utilized. In rank one the choice of scalars $Q$ does not enter the definition. Later this result was enhanced in 34 to prove the result when working over the integers. Unique decomposition for spaces of 2-morphisms follows as a corollary of these results, implying that the category $\mathcal{U}_{\mathbb{Z}}\left(\mathfrak{s l}_{2}\right)$ is Krull-Schmidt. Very recently, it was shown in [8] that $\dot{\mathcal{U}}_{\mathbb{Z}}$ also categorifies ${ }_{\mathcal{A}} \dot{\mathbf{U}}$ via the alternative trace decategorification functor Tr.

Theorem 4.2. ([34, [8]) For $\mathfrak{s l}_{2}, \mathcal{B}$ is the strongly upper triangular basis of $\dot{\mathcal{U}}_{\mathbb{Z}}$. The maps

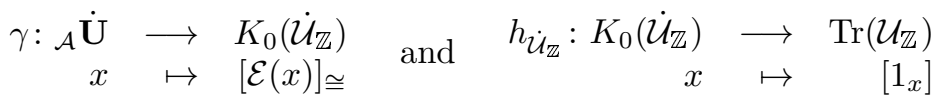

are isomorphisms of graded linear categories, providing two categorifications of ${ }_{\mathcal{A}} \mathbf{U}$.

4.5.1. Strategy of the Proof. In section 6 of [8], based on the results of [34] we give an explicit presentation of the subcategory $\left.\dot{\mathcal{U}}(n, m)_{\mathbb{Z}}\right|_{m} \mathcal{B}_{n}$ by generators and relations, which shows that this category is strongly upper triangular. Hence, the indecomposable 1 -morphisms from $\mathcal{B}$ generates $K_{0}$ and, by Proposition 3.9 , also $\operatorname{Tr}$ as $\mathbb{Z}\left[q, q^{-1}\right]$-modules. In [34] explicit isomorphisms in $\dot{\mathcal{U}}_{\mathbb{Z}}$ are given lifting the the defining relations of ${ }_{\mathcal{A}} \dot{\mathbf{U}}$, showing that $\gamma$ is an isomorphism of graded categories. Since the functor $h_{\dot{\mathcal{U}}}: K_{0}\left(\dot{\mathcal{U}}_{\mathbb{Z}}\right) \rightarrow \operatorname{Tr}\left(\dot{\mathcal{U}}_{\mathbb{Z}}\right)$ preserves composition. Finally, we use that $\operatorname{Tr}(\dot{\mathcal{U}})=\operatorname{Tr}(\mathcal{U})$, the result follows.

4.6. Two categorifications of ${ }_{\mathcal{A}} \dot{\mathbf{U}}\left(\mathfrak{s} l_{n}\right)$. Here we present the results of [33] computing $K_{0}$ and of [7] computing $\operatorname{Tr}$ for general $n$.

There is a special choice of scalars, where all $t_{i j}=1$ for $1 \leq i<n-1$. In this case, the graphical calculus is cyclic with respect to the biadjoint structure, so that isotopic diagrams represent the same 2-morphism. This will be our default choice of $Q$ in sections unless explicitly stated otherwise. We will omit explicitly mentioning $Q$ in the notation when no confusion is likely to arise. 
Theorem 4.2 was possible because the explicit isomorphisms constructed in 34 provide decompositions of arbitrary 1-morphisms into indecomposables. However, the lack of an explicit description of the canonical basis for ${ }_{\mathcal{A}} \mathbf{U}$ beyond $\mathfrak{s l}_{2}$ presents an obstacle to generalizing this result beyond $\mathfrak{s l}_{2}$. However, if one works with the 2-category $\mathcal{U}\left(\mathfrak{s l}_{n}\right)$ defined over a field $\mathbb{k}$ of characteristic 0 , then one can utilize various connections between this category and known geometric constructions to prove the following result.

Theorem 4.3. ([33], [7]) Let $\mathbb{k}$ be a field of characteristic 0 . For $\mathfrak{s l}_{n}$, the 2-category $\dot{\mathcal{U}}$ is strongly upper triangular (see Remark 15). Moreover,

$$
K_{0}(\dot{\mathcal{U}})={ }_{\mathcal{A}} \dot{\mathbf{U}}
$$

as graded linear categories and

$$
\operatorname{Tr}(\mathcal{U})=K_{0}(\dot{\mathcal{U}}) \otimes_{\mathbb{Z}} \mathbb{k}, \quad \mathrm{HH}_{i}(\mathcal{U})=0 \quad \text { for } \quad i>0 .
$$

Following a recommendation of the first author, the $\mathfrak{s l}_{3}$ case of this theorem was studied in 65.

4.6.1. Strategy of the Proof. Let us make few remarks about the proof. The result about $K_{0}$ was proved in 33. It relies on the fact that $\dot{\mathcal{U}}$ is Krull-Schmidt. To control the size of $K_{0}$ an action of $\dot{\mathcal{U}}$ was defined on the cohomology rings of partial flag varieties. Even when for $n>2$, the explicit form of the Lusztig canonical basis is not known, but the results of 61] and 63] allows us to deduce that $\dot{\mathcal{U}}$ is strongly upper triangular. The full argumentation will be given in [7].

4.6.2. Applications. In [40, 54] it was shown that $\mathfrak{s l}_{n}$ link homology theories can be obtained from $\dot{\mathcal{U}}\left(\mathfrak{s l}_{m}\right)$ by means of the so-called foamation functors $F$, first studied in [48. More precisely, there is an extended version 2BFoam of the Bar-Natan foam category introduced by Blanchet [9]. (The advantage of Blanchet's version is that it is functorial with respect to link cobordisms, when Bar-Natan version is functorial up to sign.) In [40, 54] an analog $n$ BFoam of the Blanchet foam category for $\mathfrak{s l}_{2}$ is defined for $\mathfrak{s l}_{n}$ and families of 2-representations $F$ for were defined from $\dot{\mathcal{U}}\left(\mathfrak{s l}_{m}\right)$ into the foam categories $n$ BFoam.

A general procedure was laid out by Cautis [17] using categorical skew Howe duality to pull back complexes associated to a tangle to a complex in $\operatorname{Kom}(\dot{\mathcal{U}})$. Combining this with the foamation functors allows one to pull back the complexes of foams associated to a tangle to a complex in $\operatorname{Kom}(\dot{\mathcal{U}})$. It was shown that all of the foam relations used for $\mathfrak{s l}_{n}$-link homology arise from relations in $\dot{\mathcal{U}}\left(\mathfrak{s l}_{m}\right)$ via foamation functors. These foamation functors categorify a skew Howe functor defined at the decategorified level by Cautis, Kamnitzer, and Morrison that realize web relations from relations in the quantum group $\dot{\mathbf{U}}_{q}\left(\mathfrak{s l}_{m}\right)[18$.

Recall that we have two flavors of Euler characteristic associated with $K_{0}$ and $\mathrm{Tr}$,

$$
\begin{aligned}
\chi_{\operatorname{tr}}: & \operatorname{Kom}(\mathrm{F}(\mathcal{U})) \rightarrow \operatorname{Tr}(\mathrm{F}(\mathcal{U})) \\
\chi: & \operatorname{Kom}(\mathrm{F}(\dot{\mathcal{U}})) \rightarrow \mathrm{K}_{0}(\mathrm{~F}(\dot{\mathcal{U}})) .
\end{aligned}
$$

Below is one of the applications of Theorem 4.3.

Corollary 4.4. In $\mathfrak{s l}_{n}$ link homologies theories, we have

$$
\chi_{t r}=\chi \text {. }
$$

Remark 20. The $\mathfrak{s l}_{2}$ case of Corollary 4.4 was first proven by Cooper-Krushkal [21, Section 3].

Proof. Let $\mathcal{C}:=F\left(\dot{\mathcal{U}}\left(\mathfrak{s l}_{m}\right)\right)$. We have the Chern character map $h_{\mathcal{C}}: K_{0}(\mathcal{C}) \rightarrow \operatorname{Tr}(\mathcal{C})$ sending $[x] \cong \rightarrow\left[1_{x}\right]$. The induced map $\chi \rightarrow \chi_{\operatorname{tr}}$ is surjective, since $\chi_{\mathrm{tr}}$ uses trace classes of the identity maps only. It remains to show that this map is injective. Note that $K_{0}(\dot{\mathcal{U}})$ is freely generated by indecomposables and $h_{\dot{\mathcal{U}}}$ is an isomorphism after tensoring with any field of zero characteristic by Theorem 4.3, so $h_{\dot{\mathcal{U}}}$ is injective. Hence, $F\left(K_{0}(\dot{\mathcal{U}})\right)=F\left(h_{\dot{\mathcal{U}}}\left(K_{0}(\dot{\mathcal{U}})\right)\right)$. The fact that any linear functor commutes with $K_{0}$ implies the result. 


\section{Graded traces of CATEgorified QUANTUM Groups}

By allowing homogeneous, but not necessarily grading preserving 2-morphisms in $\mathcal{U}(\mathfrak{g})$ we can define a version of the 2-category with larger 2-hom spaces. Let $\mathcal{U}^{*}(\mathfrak{g})$ denote the 2-category with the same objects and the same 1-morphisms as $\mathcal{U}(\mathfrak{g})$, but with 2-hom spaces between one morphisms $X \mathbf{1}_{\lambda}$ and $Y \mathbf{1}_{\lambda}$ given by

$$
\mathcal{U}^{*}(\mathfrak{g})\left(X \mathbf{1}_{\lambda}, Y \mathbf{1}_{\lambda}\right):=\bigoplus_{t \in \mathbb{Z}} \mathcal{U}(\mathfrak{g})\left(X \mathbf{1}_{\lambda}, Y \mathbf{1}_{\lambda}\langle t\rangle\right) .
$$

One can alternatively think of $\mathcal{U}^{*}$ as the result of adding isomorphisms $X \mathbf{1}_{\lambda} \rightarrow X \mathbf{1}_{\lambda}\langle t\rangle$ for all $t \in \mathbb{Z}$. This essentially kills the grading making Grothendieck ring $K_{0}\left(\mathcal{U}^{*}\right)$ only a $\mathbb{Z}$-module, rather than a $\mathbb{Z}\left[q, q^{-1}\right]$-module. While this version of the 2 -category is less interesting from a $K_{0}$ perspective, it has interesting consequences for the trace.

5.1. The trace of 2-category $\mathcal{U}^{*}\left(\mathfrak{s l}_{2}\right)$ and the current algebra of $\mathfrak{s l}_{2}$. In this section we outline the results of $[8]$. We work with $\mathfrak{g}=\mathfrak{s l}_{2}$ and our 2-morphisms are defined over $\mathbb{Z}$.

Let us consider the algebra $\mathfrak{s l}_{2}[t]=\mathfrak{s l}_{2} \otimes \mathbb{Q}[t]$. As a $\mathbb{Q}$-algebra, its universal enveloping algebra $\mathbf{U}\left(\mathfrak{s l}_{2}[t]\right)$ is generated by $E_{i}, F_{i}$ and $H_{i}$ for $i \geq 0$, where $X_{i}=X \otimes t^{i}$, subject to the following relations:

$$
\begin{gathered}
{\left[H_{i}, H_{j}\right]=\left[E_{i}, E_{j}\right]=\left[F_{i}, F_{j}\right]=0} \\
{\left[H_{i}, E_{j}\right]=2 E_{i+j}, \quad\left[H_{i}, F_{j}\right]=-2 F_{i+j}, \quad\left[E_{i}, F_{j}\right]=H_{i+j} .}
\end{gathered}
$$

An integral basis of $\mathbf{U}\left(\mathfrak{s l}_{2}[t]\right)$ was constructed in $[23$ and it is the lifting of the integral PoincareBirkhoff-Witt (PBW) type basis. The following is one of the main results of [8].

Theorem 5.1. There is an isomorphism of additive categories

$$
h: \dot{\mathbf{U}}\left(\mathfrak{s l}_{2}[t]\right) \longrightarrow \operatorname{Tr}\left(\mathcal{U}_{\mathbb{Z}}^{*}\left(\mathfrak{s l}_{2}\right)\right)
$$

where $\dot{\mathbf{U}}\left(\mathfrak{s l}_{2}[t]\right)$ is the idempotented integral form of the current algebra for $\mathfrak{s l}_{2}$.

5.1.1. Sketch of the Proof. To define an isomorphism $h: \dot{\mathbf{U}}\left(\mathfrak{s l}_{2}[t]\right) \longrightarrow \operatorname{Tr}\left(\mathcal{U}_{\mathbb{Z}}^{*}\left(\mathfrak{s l}_{2}\right)\right)$ we must specify where the generating elements of $\dot{\mathbf{U}}\left(\mathfrak{s l}_{2}[t]\right)$ are sent. To define this map over the integers as was done in 8 we must work with the integral basis of the current algebra introduced by Garland. However, to illustrate the central ideas of this theorem we will explain which elements of the trace correspond to the current algebra generators $E_{r}=E \otimes t^{r}, F_{s}=F \otimes t^{s}$, and $H_{k}=H \otimes t^{k}$. We illustrate these assignments below:

$$
E_{r} 1_{\lambda} \mapsto\left[\oint^{r}\right] \quad F_{s} 1_{\lambda} \mapsto\left[\wp^{s}\right] \quad H_{r} 1_{\lambda} \mapsto\left[p_{r}(\lambda) \operatorname{Id}_{\mathbf{1}_{\lambda}}\right]
$$

where $p_{r}(\lambda)$ is the degree $r$ endomorphism of $\mathbf{1}_{\lambda}$ given by the power sum symmetric function defined in section 4.3 and $p_{0}(\lambda)$ is $\lambda$.

In order to verify that these assignments define a homomorphism we must verify the current algebra axioms. Since our target is the trace of the 2-category $\mathcal{U}_{\mathbb{Z}}^{*}\left(\mathfrak{s l}_{2}\right)$, we perform these calculations in the graphical calculus as though each diagram was on an annulus. For example, the most interesting relation $\left[E_{r}, F_{s}\right]=H_{r+s}$ follows from the computation achieved by twice utilizing the most sophisticated 
$\operatorname{sl}(2)$-relations (4.26) and (4.28):

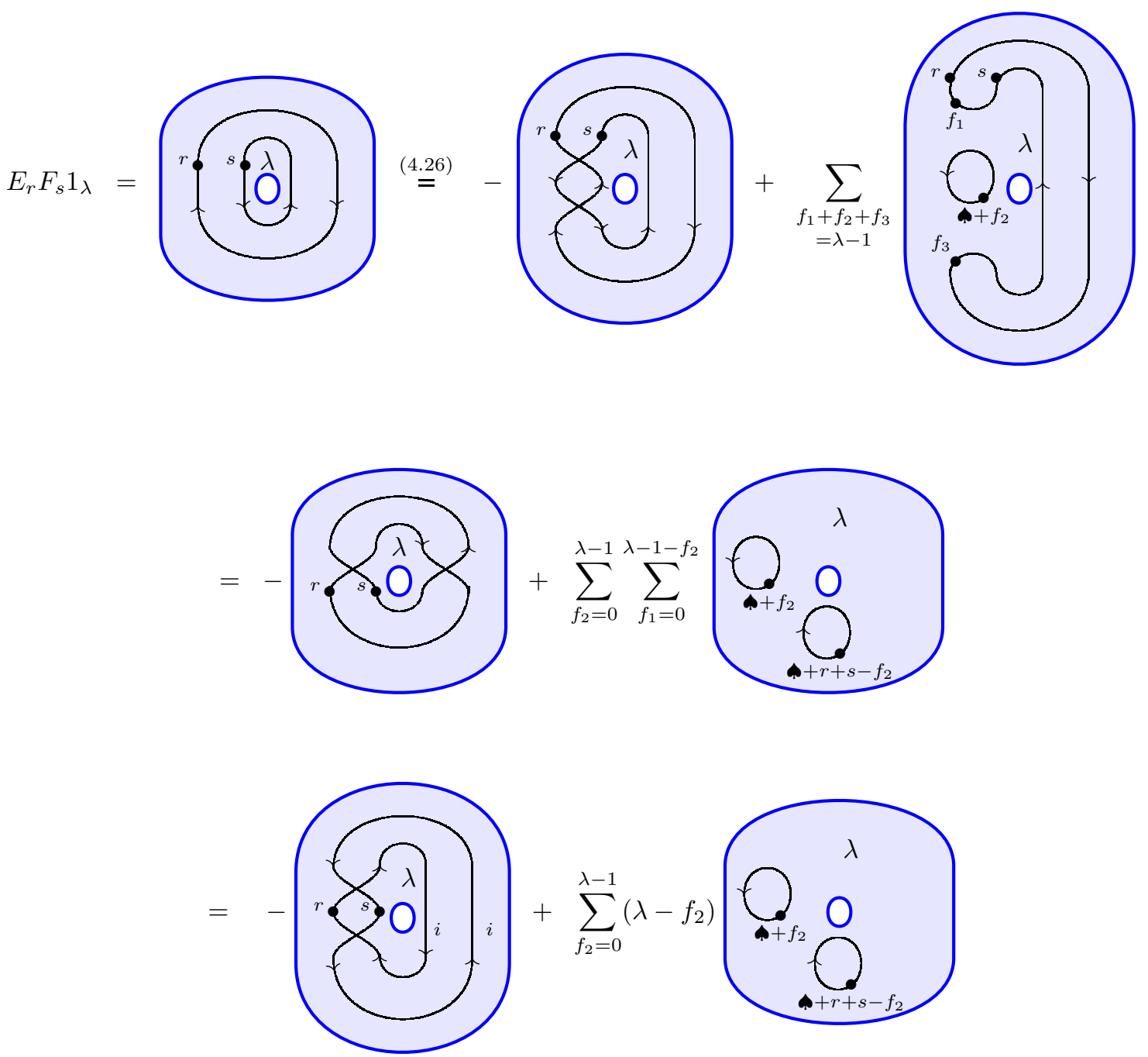

Using the nilHecke relations and the identity decomposition equation it is not hard to check that

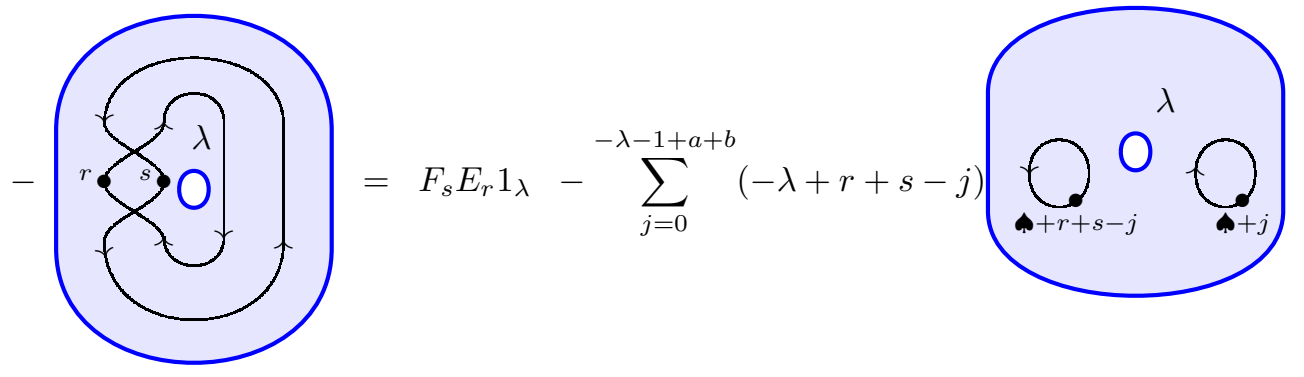


Therefore it suffices to show that the action of $H_{r+s}$ is equal to

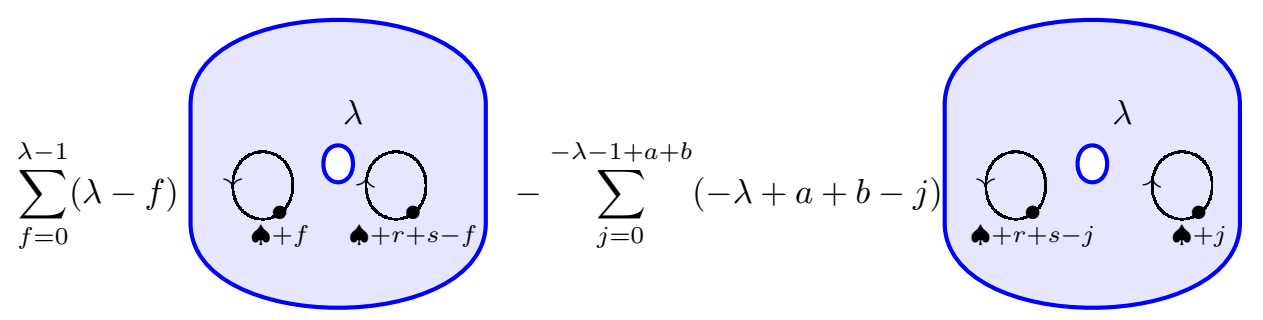

The first summation is zero when $\lambda-1<0$ and the second summation is zero when $-\lambda-1+a+b<0$.

We prove the claim by considering four cases:

- Case 1: $r+s=0$.

- Case 2: $r+s>0, \lambda-1 \geq 0$ and $-\lambda-1+r+s<0$.

- Case 3: $r+s>0, \lambda-1<0$ and $-\lambda-1+r+s \geq 0$.

- Case 4: $r+s>0, \lambda-1 \geq 0$ and $-\lambda-1+r+s \geq 0$.

Case 1 is easy to verify. For cases $2-4$ we show that (5.2) is equal to

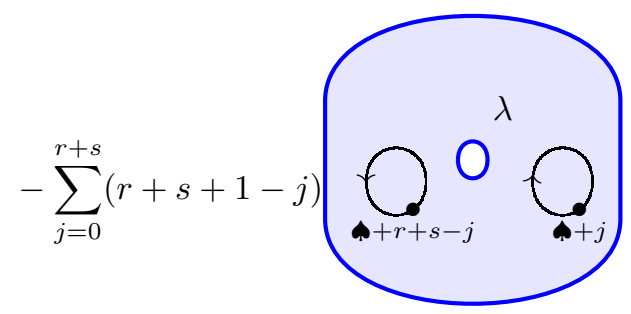

by adding 0 in the form of the infinite Grassmannian relation (4.24). For case 2 , since $\lambda \geq 1$ we can add zero in the form

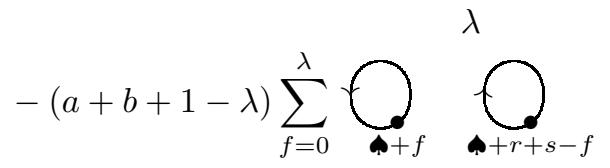

to (5.2) and get (5.3). For case 3, we add

$$
-(\lambda+1) \sum_{j=0}^{-\lambda+r+s} \bigodot_{\infty+r+s-j} \bigcap_{s+j}
$$

and for case 4 we re-index the first summation setting $j=r+s-f$, combine the terms, and add

$$
-(\lambda+1) \sum_{j=0}^{r+s} \bigcap_{\omega+r+s-j}
$$

Once we have defined a homomorphism $h: \dot{\mathbf{U}}\left(\mathfrak{s l}_{2}[t]\right) \longrightarrow \operatorname{Tr}\left(\mathcal{U}_{\mathbb{Z}}^{*}\left(\mathfrak{s l}_{2}\right)\right)$, establishing that the map is an isomorphism utilizes the following Lemma.

Lemma 21. Let $\mathcal{C}$ be a linear category. Let $H:=\bigoplus_{x \in \mathrm{Ob}(\mathcal{C})} \mathcal{C}(x, x)$, and let $K \subset H$ be a subgroup. Assume that there is a linear map $\pi: H \rightarrow K$ with the following properties: 
(1) $\pi$ is a projection,

(2) $[\pi(f)]=[f]$ for every $f \in H$, and

(3) for every $g \in \mathcal{C}(x, y)$ and $h \in \mathcal{C}(y, x)(x, y \in \mathrm{Ob}(\mathcal{C})), \pi(g h)=\pi(h g)$;

then $\operatorname{Tr}(\mathcal{C})$ is isomorphic to $K$.

The two main ingredients in establishing that $h$ is a homomorphism are

- identification of a certain subset $K$ of 2-endomorphisms in $\mathcal{U}$ with the Garland integral form of the positive part of the loop algebra (or the current algebra),

- construction of the projection $\pi$ satisfying conditions of the lemma.

To solve the first problem, we explicitly construct generators of $\operatorname{Tr}\left(\mathcal{U}^{*}\right)$ and compute relations between them. Then we identify this PBW type basis with Garland's integral form.

5.2. The current algebra for $\mathfrak{s l}_{n}$. We expect to be able to generalize Theorem 5.1 to $\mathfrak{s l}_{n}$.

Let us define the current algebra $\mathbf{U}\left(\mathfrak{s l}_{n}[t]\right)=\mathbf{U}\left(\mathfrak{s l}_{n} \otimes \mathbb{Q}[t]\right)$. This algebra is generated over $\mathbb{k}$ by $x_{i, r}^{+}, x_{i, s}^{-}$and $\xi_{i, k}$ for $r, s, k \in \mathbb{N} \cup\{0\}$ and $1 \leq i<n$ modulo the following relations:

C1: For $i, j$ in $I$ and $r, s \in \mathbb{N} \cup\{0\}$

$$
\left[\xi_{i, r}, \xi_{j, s}\right]=0
$$

C2: For any $i, j \in I$ and $k \in \mathbb{N} \cup\{0\}$,

$$
\left[\xi_{i, 0}, x_{j, k}^{ \pm}\right]= \pm a_{i j} x_{j, k}^{ \pm}
$$

C3: For any $i, j \in I$ and $r, k \in \mathbb{N} \cup\{0\}$,

$$
\left[\xi_{i, r}, x_{j, k}^{ \pm}\right]= \pm a_{i j} x_{j, r+k}^{ \pm}
$$

C4: For $i, j \in I$ and $k, \ell \in \mathbb{N} \cup\{0\}$

$$
\left[x_{i, k+1}^{ \pm}, x_{j, \ell}^{ \pm}\right]=\left[x_{i, k}^{ \pm}, x_{j, \ell+1}^{ \pm}\right]
$$

C5: For $i, j \in I$ and $k, \ell \in \mathbb{N} \cup\{0\}$

$$
\left[x_{i, k}^{+}, x_{j, \ell}^{-}\right]=\delta_{i, j} \xi_{i, k+\ell}
$$

C6: Let $i \neq j$ and set $m=1-a_{i j}$. For every $k_{1}, \ldots, k_{m} \in \mathbb{N} \cup\{0\}$ and $\ell \in \mathbb{N} \cup\{0\}$

$$
\sum_{\pi \in S_{m}} \sum_{s=0}^{m}(-1)^{s}\left(\begin{array}{c}
m \\
s
\end{array}\right) x_{i, k_{\pi(1)}}^{ \pm} \ldots x_{i, k_{\pi(s)}}^{ \pm} x_{j, \ell}^{ \pm} x_{i, k_{\pi(s+1)}}^{ \pm} \ldots x_{i, k_{\pi(m)}}^{ \pm}=0 .
$$

For more on the relationship between the current algebra and specializations of the Yangian see 3 . Recall that we denote by $\dot{\mathbf{U}}\left(\mathfrak{s l}_{n}[t]\right)$ the idempotented form of the current algebra.

Exercise 22. Show for $t=1$ that the current algebra relations restrict to the defining relations for $\mathbf{U}\left(\mathfrak{s l}_{n}\right)$.

The next proposition is a step towards generalizing Theorem 5.1

Proposition 23. Let $\mathrm{E}_{i, r}, \mathrm{~F}_{j, s}, \mathrm{H}_{i, r}$ denote the generators of $\operatorname{Tr}\left(\mathcal{U}^{*}\left(\mathfrak{s l}_{n}\right)\right)$ :

$$
\mathrm{E}_{i, r} 1_{\lambda}:=\left[\begin{array}{ll}
f_{r} & \lambda \\
i &
\end{array}\right], \quad \mathrm{F}_{j, s} 1_{\lambda}:=\left[\begin{array}{ll}
f_{s} & \lambda \\
j &
\end{array}\right], \quad \mathrm{H}_{i, r} 1_{\lambda}:=\left[p_{i, r}(\lambda) \operatorname{Id}_{\mathbf{1}_{\lambda}}\right],
$$

where $p_{i, r}(\lambda)$ was defined in equation [4.36. For any choice of scalars $Q$ with $t_{i j}^{2}=t_{j i}^{2}=t_{i j} j^{-1} t_{j i}=1$ for $i \neq j$, there is a well defined homomorphism

$$
h: \dot{\mathbf{U}}\left(\mathfrak{s l}_{n}[t]\right) \longrightarrow \operatorname{Tr}\left(\mathcal{U}^{*}\left(\mathfrak{s l}_{n}\right)\right),
$$


given by

$$
x_{i, r}^{+} 1_{\lambda} \mapsto(-1)^{(i+1) r} \mathrm{E}_{i, r} 1_{\lambda}, \quad x_{j, s}^{-} 1_{\lambda} \mapsto(-1)^{(j+1) s} \mathrm{~F}_{j, s} 1_{\lambda}, \quad \xi_{i, r} 1_{\lambda} \mapsto(-1)^{(i+1) r} \mathrm{H}_{i, r} 1_{\lambda} .
$$

Proof. To prove this proposition we verify the current algebra relations using the relations in the 2category $\mathcal{U}^{*}\left(\mathfrak{s l}_{n}\right)$. We only need to consider the case $i \neq j$, since the relations in $\mathcal{U}^{*}\left(\mathfrak{s l}_{2}\right)$ have been proven in [8. $\mathbf{C 1}$ is clear, since multiplication by a bubble is a commutative operation. We prove the equality C3 for the case $j=i \pm 1$. For convenience we will only depict the interior of the annulus for diagrams on the annulus in our calculation below where $v_{i j}:=t_{i j}^{-1} t_{j i}$.

$$
\mathrm{H}_{i, a} \mathrm{E}_{j, b} 1_{\lambda}=\sum_{k=0}^{a} k \bigodot_{a+k}^{i} \bigodot_{\rightarrow+a-k}^{j} \underbrace{\lambda}_{b}
$$
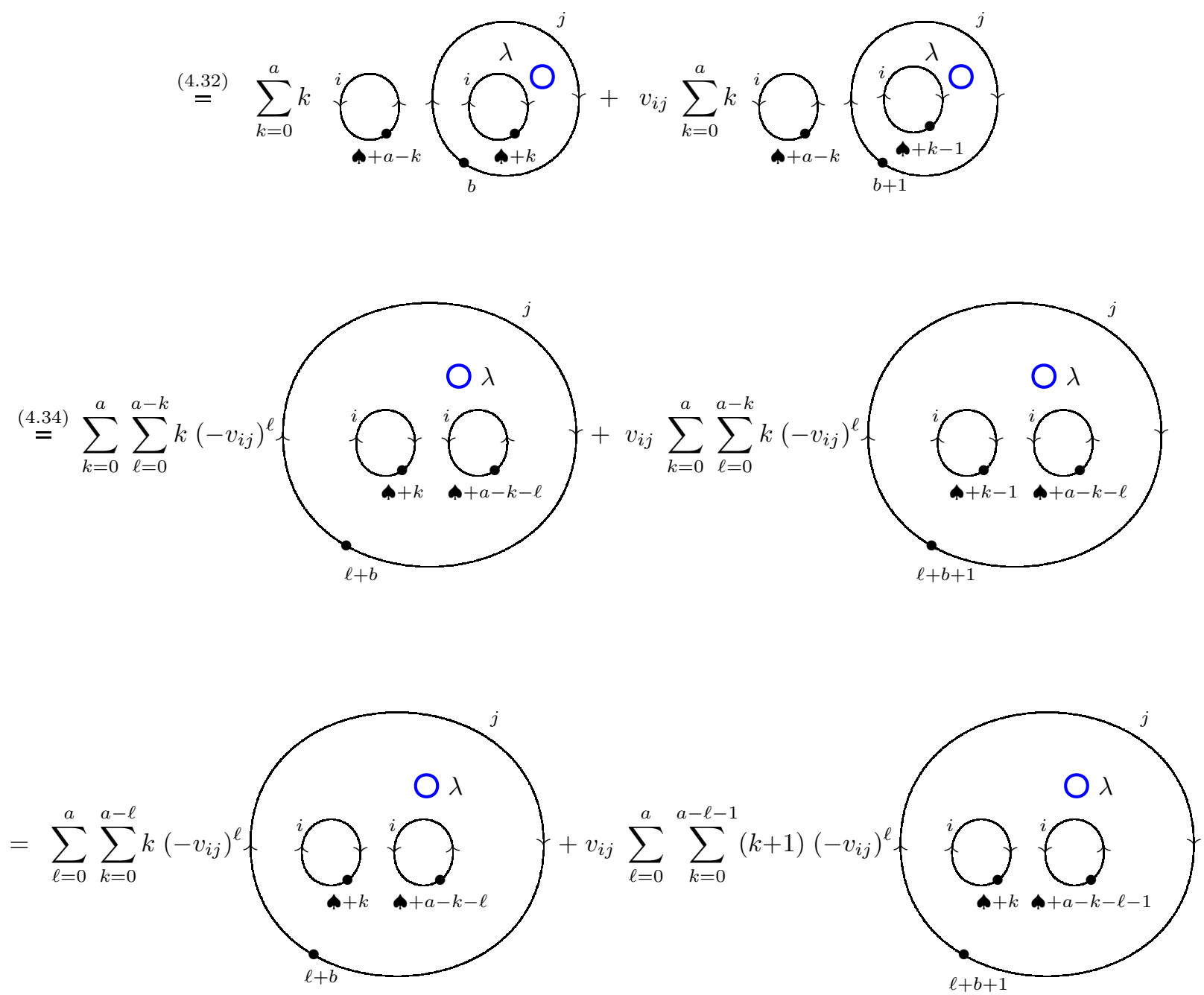


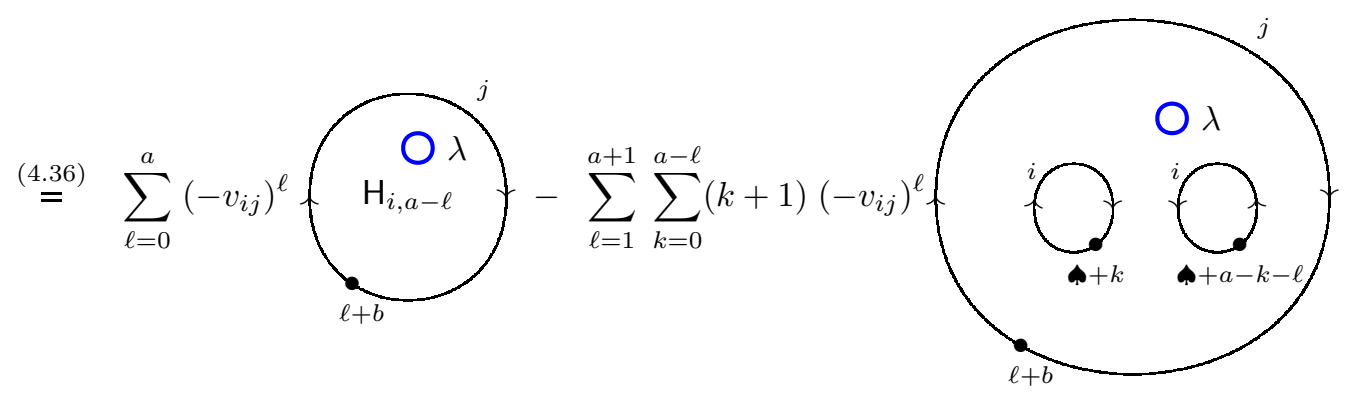

Pulling off $k$ of the summands in the second term and observing that the $\ell=a+1$ term vanishes we have

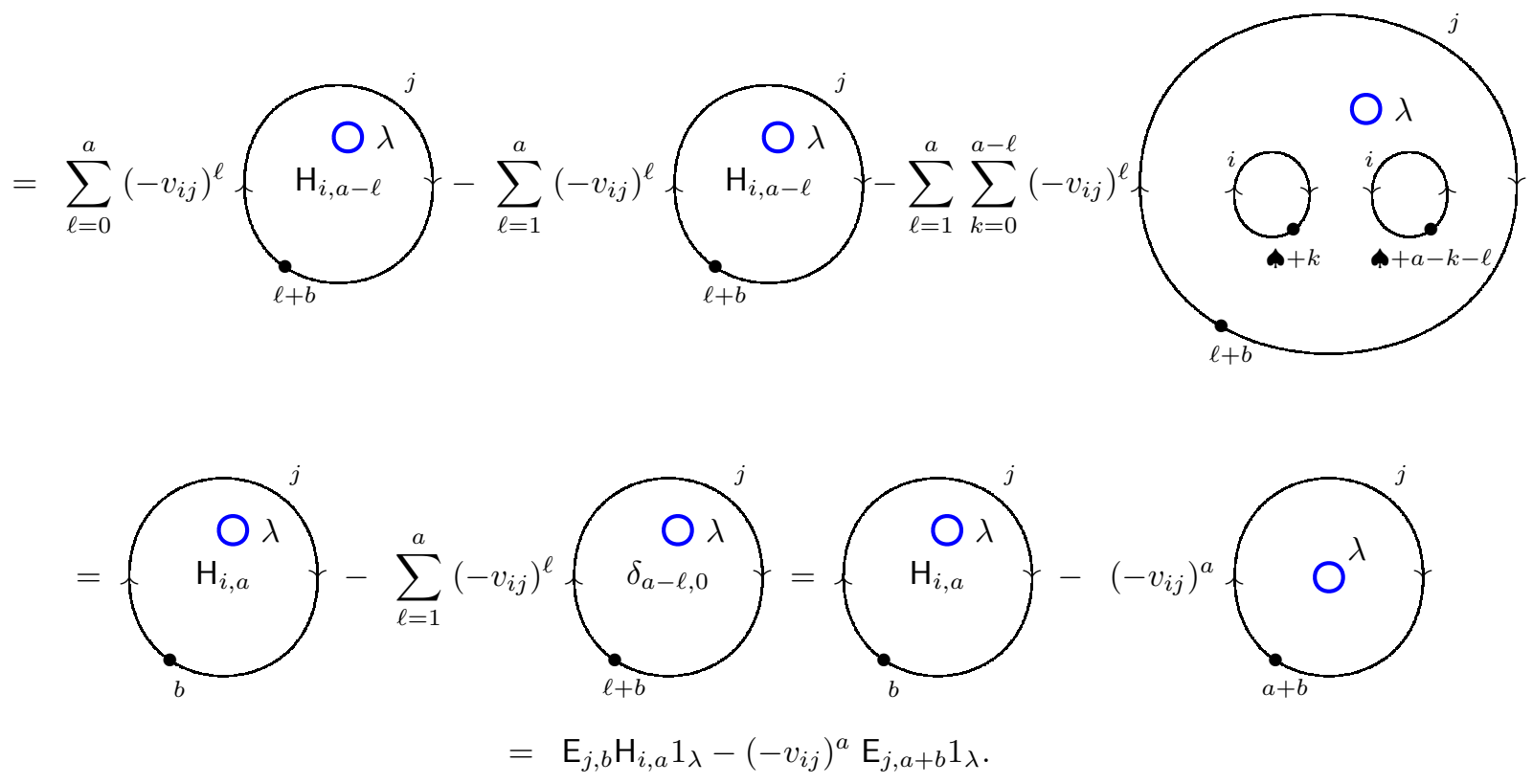

By choosing $v_{i j}=1$, we get

$$
\left[\mathrm{H}_{i, a}, \mathrm{E}_{j, b}\right] 1_{\lambda}=-(-1)^{a} \mathrm{E}_{j, a+b} 1_{\lambda} .
$$

The relation

$$
\left[\mathrm{H}_{i, a}, \mathrm{~F}_{j, b}\right] 1_{\lambda}=(-1)^{a} \mathrm{~F}_{j, a+b} 1_{\lambda} .
$$

can be proven in a similar way. $\mathbf{C 4}$ follows from the relations (4.16) and (4.17):
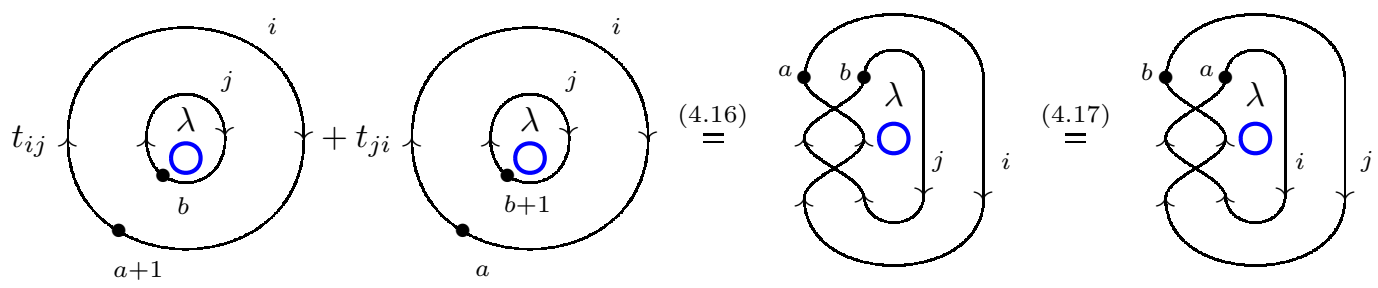


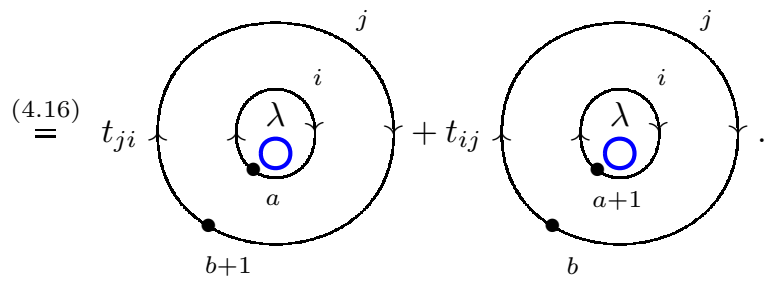

Dividing both sides of this equation by $t_{i j}$, and by choosing $v_{i j}=1$, we get

$$
\left[\mathrm{E}_{i, a+1}, \mathrm{E}_{j, b}\right] 1_{\lambda}=-\left[\mathrm{E}_{i, a}, \mathrm{E}_{j, b+1}\right] 1_{\lambda} .
$$

If we reverse the arrows in the preceding equation, they still hold:

$$
\left[\mathrm{F}_{i, a+1}, \mathrm{~F}_{j, b}\right] 1_{\lambda}=-\left[\mathrm{F}_{i, a}, \mathrm{~F}_{j, b+1}\right] 1_{\lambda} .
$$

The choice of signs in (5.8) correspond to

$$
\left[x_{i, a+1}^{ \pm}, x_{j, b}^{ \pm}\right]=\left[x_{i, a}^{ \pm}, x_{j, b+1}^{ \pm}\right] .
$$

The relation $\mathbf{C 5}$ for the case $i \neq j$ can be checked the following way:
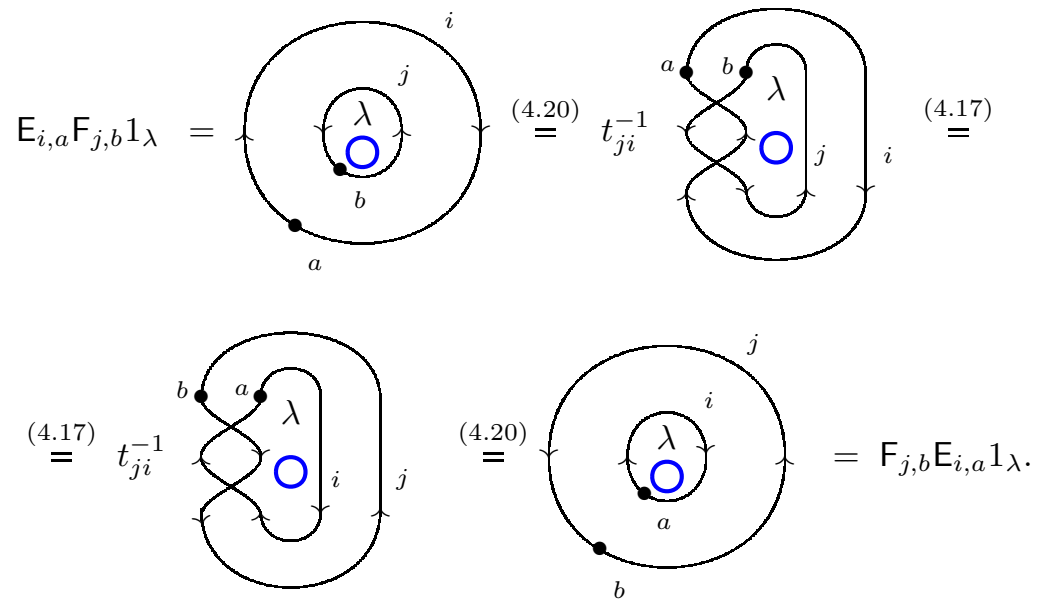

We now prove the Serre relation $\mathbf{C 6}$ in the case when $i \cdot j=-1$. Let us prove the identity

$$
\mathrm{E}_{i, a} \mathrm{E}_{j, b} \mathrm{E}_{i, c} 1_{\lambda}+\mathrm{E}_{i, c} \mathrm{E}_{j, b} \mathrm{E}_{i, a} 1_{\lambda}=\mathrm{E}_{i, a} \mathrm{E}_{i, c} \mathrm{E}_{j, b} 1_{\lambda}+\mathrm{E}_{j, b} \mathrm{E}_{i, a} \mathrm{E}_{i, c} 1_{\lambda} .
$$
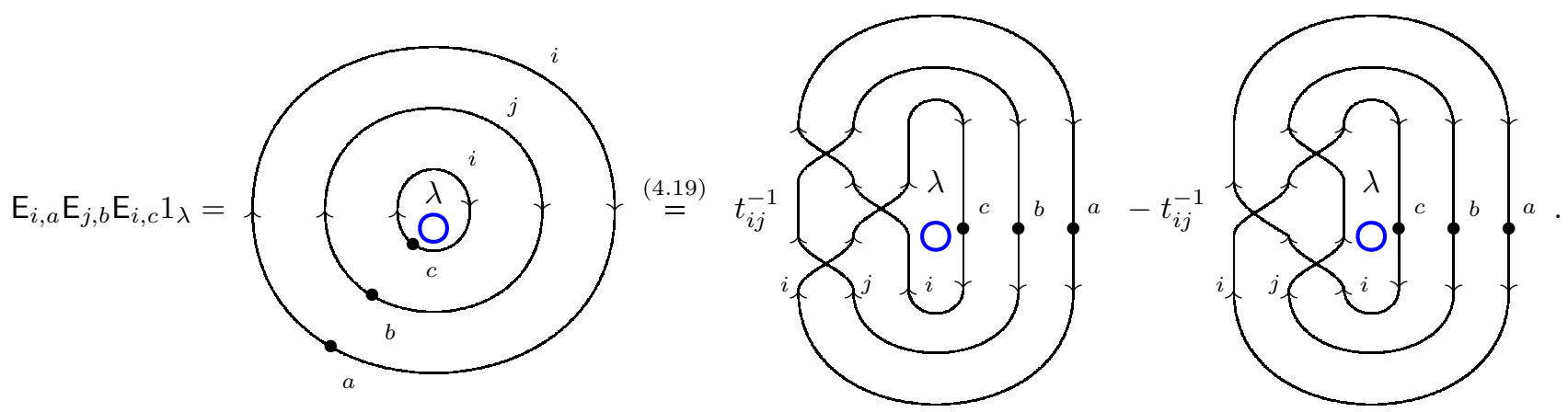
The first term on the right-hand-side of (5.9) can be simplified

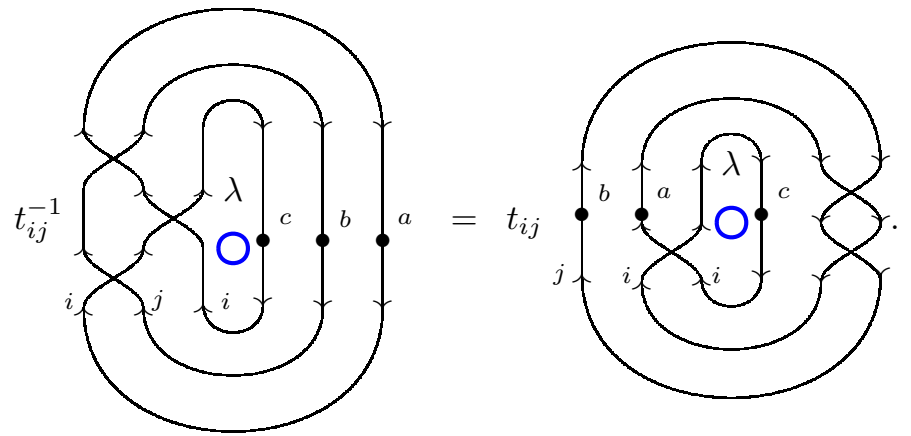

using the biadjoint relations and equations (4.14) and (4.15) to slide crossings around the annulus. This diagram can be further simplified using the KLR relations

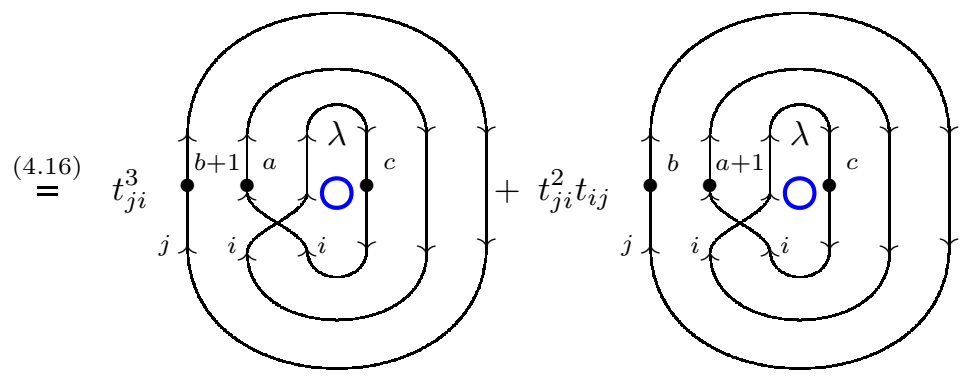

where we freely slide dots through caps and cups using the dot cyclicity relation (4.12). The second term on the right-hand-side of (5.9) can also be simplified

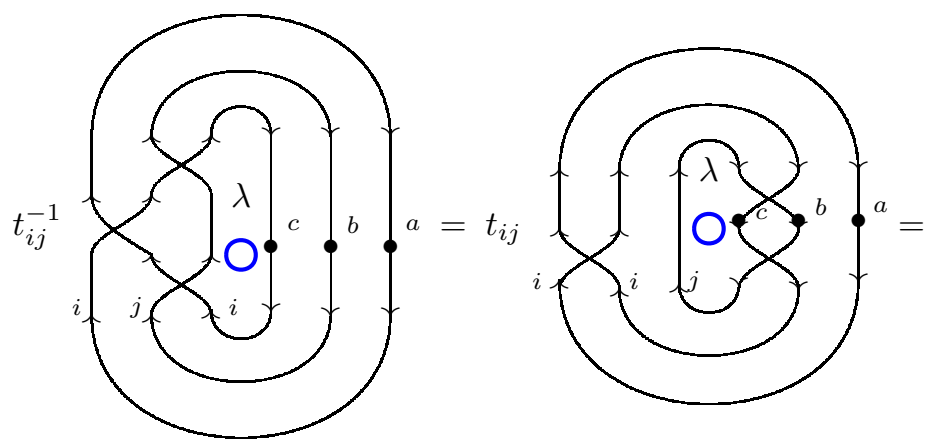




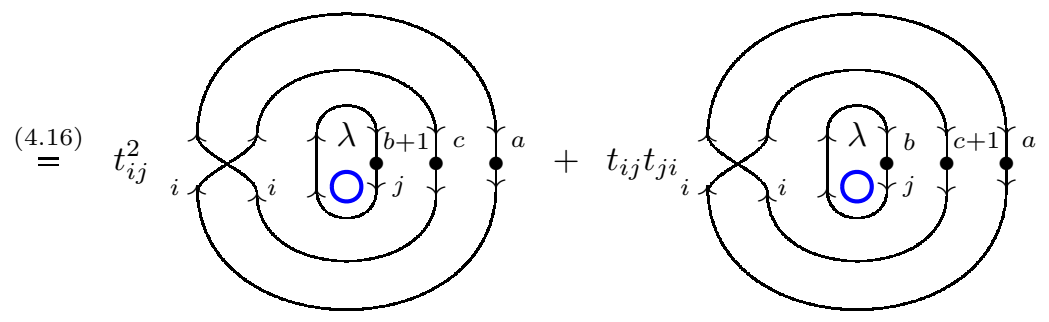

Combining terms and simplifying using that $t_{i j}^{2}=t_{j i}^{2}=1, v_{i j}=1$ for our choice of scalars, we have
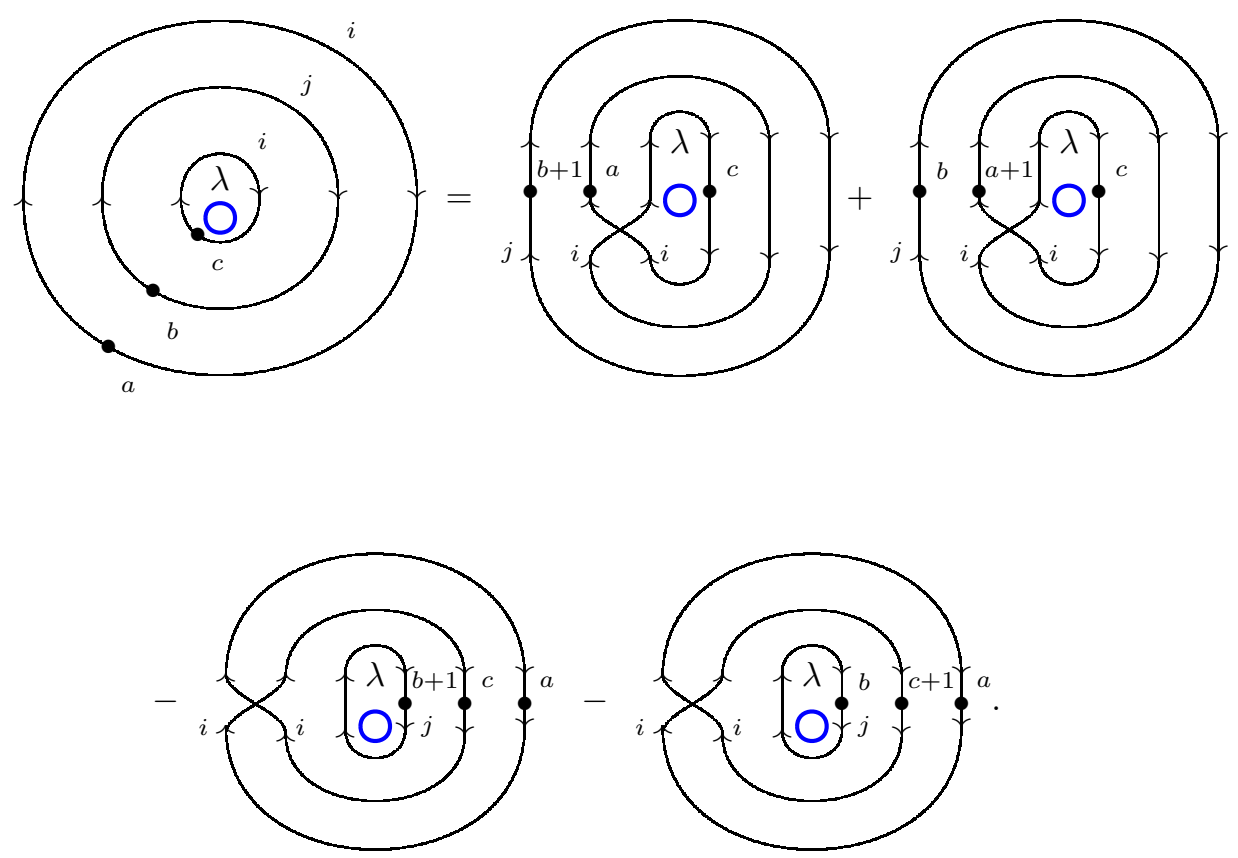

By interchanging $a$ and $c$ in the equation (5.12), we get

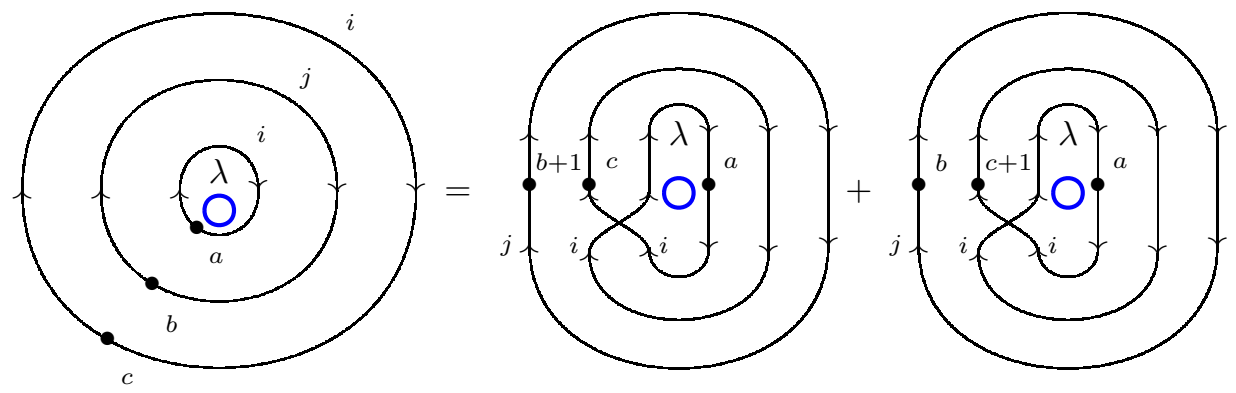




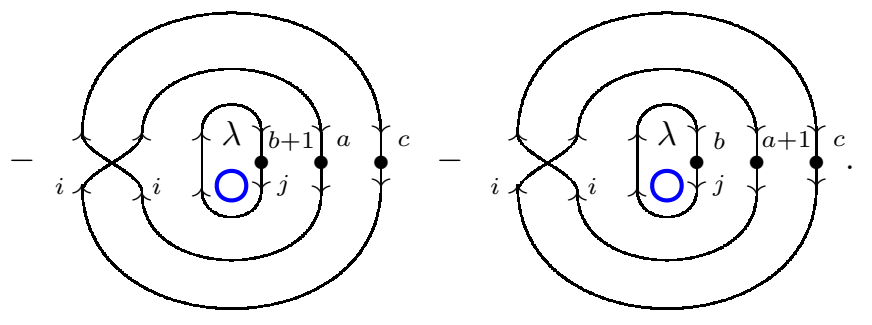

Using the relation (2.7) and induction, one can easily prove the following equation:

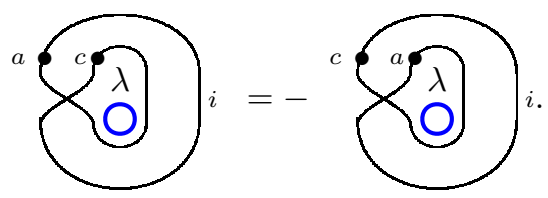

Also, the following equation directly follows from (2.4) and (5.14)

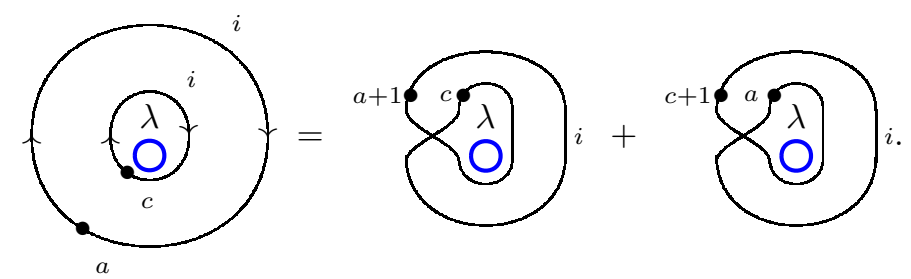

We add the right and left hand sides of the equations (5.12) and (5.13), and after eliminating terms using (5.14), we get

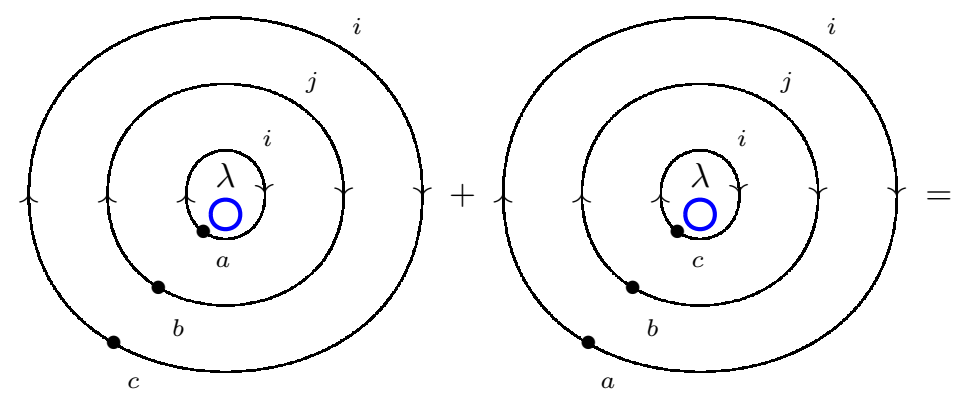



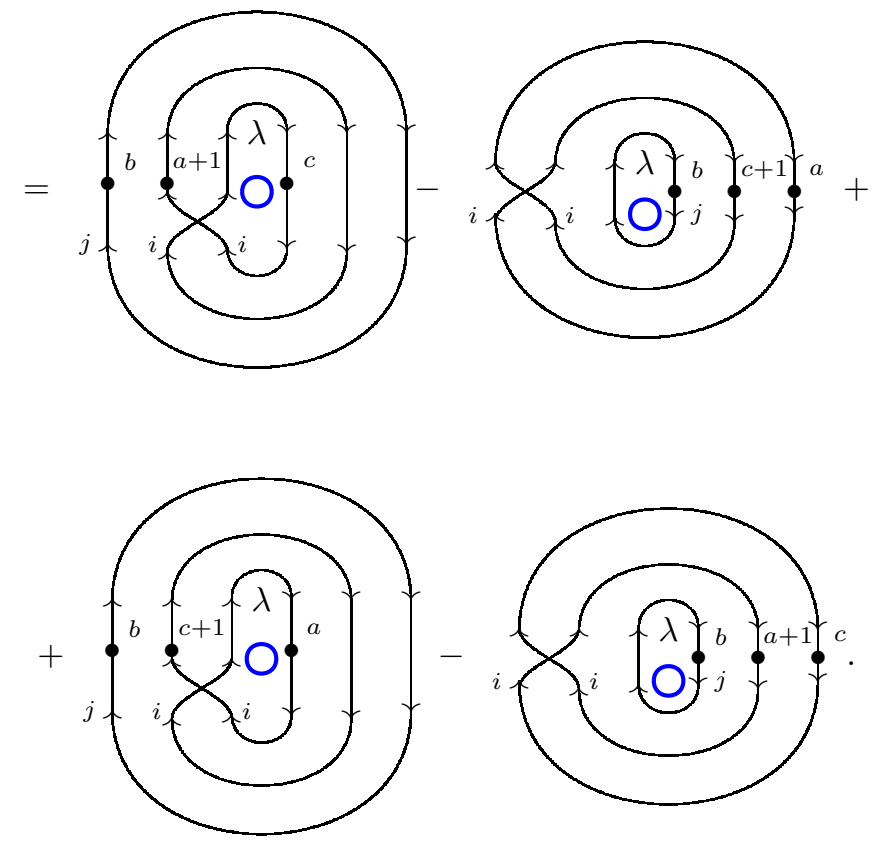

Combining the respective terms using the relation (5.15) in the equation (5.16), we have
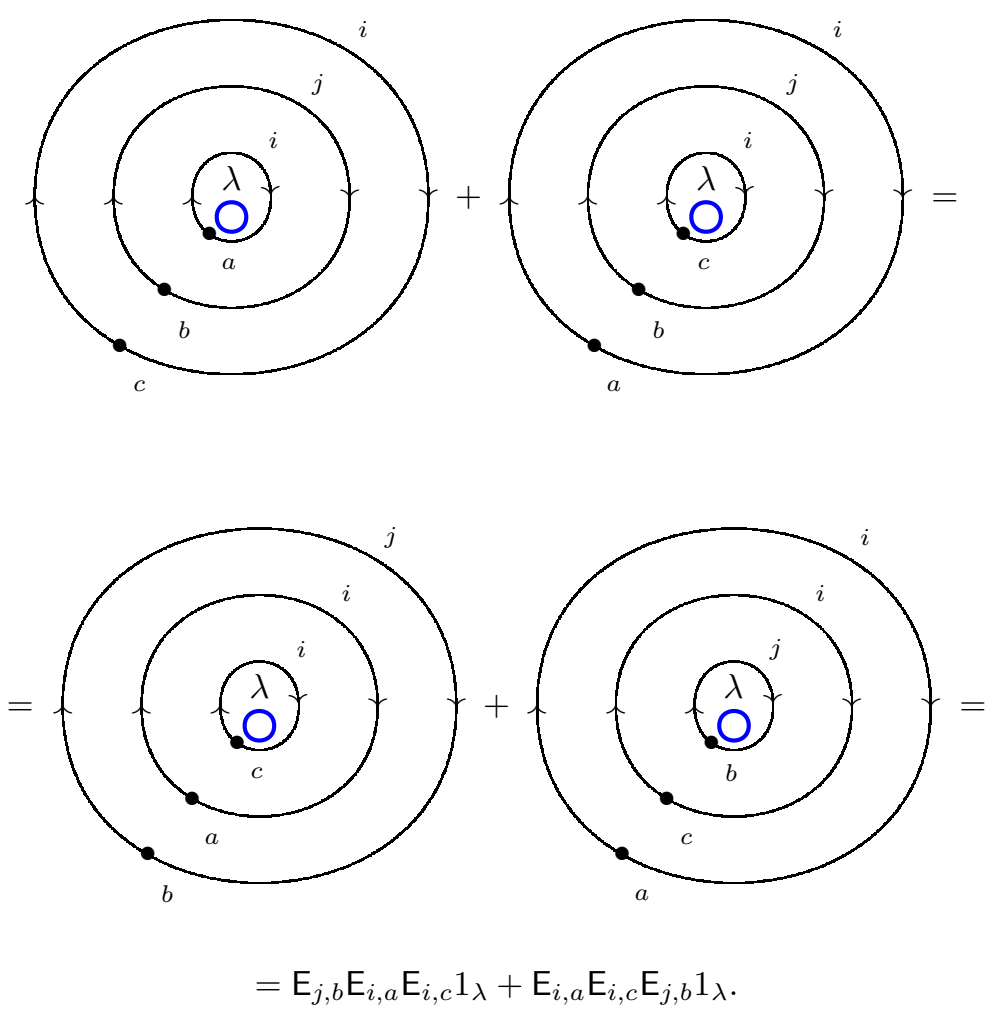
Reversing the orientation of the arrows, we get the identity

$$
\mathrm{F}_{i, a} \mathrm{~F}_{j, b} \mathrm{~F}_{i, c} 1_{\lambda}+\mathrm{F}_{i, c} \mathrm{~F}_{j, b} \mathrm{~F}_{i, a} 1_{\lambda}=\mathrm{F}_{i, a} \mathrm{~F}_{i, c} \mathrm{~F}_{j, b} 1_{\lambda}+\mathrm{F}_{j, b} \mathrm{~F}_{i, a} \mathrm{~F}_{i, c} 1_{\lambda}
$$

\section{ACtion On CEnter of OBJECts in $\dot{\mathcal{U}}$}

This section is devoted to the proof of Theorem 1.3. The action of the current algebra on the trace of any 2-representation follows directly from the functotiality of the trace. The rest of the section establishes the action on the center.

6.0.1. Centers of categories. The center $Z(\mathcal{C})$ of an additive category $\mathcal{C}$ is the endomorphism ring of the identity functor $\operatorname{Id}_{\mathcal{C}}$ on $\mathcal{C}$. Note that an endomorphism of $\operatorname{Id}_{\mathcal{C}}$ is a natural transformation $\alpha: \operatorname{Id}_{\mathcal{C}} \Rightarrow \operatorname{Id}_{\mathcal{C}}$. Such a natural transformation consists of a map $\alpha_{x}: x \rightarrow x$ for each object $x$ of $\mathcal{C}$ satisfying the requirement that for any map $f: x \rightarrow y$ in $\mathcal{C}$, we get a commutative square

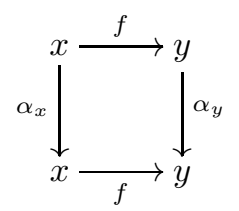

Exercise 24. Show that $Z(\mathcal{C})$ is commutative ring. Hint: use the Eckmann-Hilton argument.

An additive category is an object of the linear 2-category AdCat of additive categories, additive functors, and natural transformations. For an arbitrary, linear 2-category $\mathbf{C}$ we define the center $Z(x)$ of an object $x \in \mathrm{Ob}(\mathbf{C})$ as the ring of endomorphisms $\mathbf{C}\left(1_{x}, 1_{x}\right)$, see [22]. Define the center of objects of the 2-category $\mathbf{C}$ as the $Z(\mathbf{C})=\bigoplus_{x \in \mathrm{Ob}(\mathbf{C})} Z(x)$.

The relationship between Hochschild homology and cohomology described in section 2.2 .3 can now be recast in a more general framework.

Exercise 25. Let $\mathbf{C}$ be a linear 2-category $\mathbf{C}$. Show that for each $x \in \mathrm{Ob}(\mathbf{C})$, the abelian group $\operatorname{Tr}(\mathbf{C}(x, x))$ is a module over the ring $Z(x)$.

Example 12. In the 2-category $\mathcal{U}\left(\mathfrak{s l}_{2}\right)$, an element in the center $Z(\lambda)$ for an object $\lambda \in \operatorname{Ob}\left(\mathcal{U}\left(\mathfrak{s l}_{2}\right)\right)$ is given by any closed diagram in the graphical calculus. It was shown in Section 8 of [37] that any such closed diagram can be reduced to a product of non-nested dotted bubbles with the same orientation. As explained in section 4.3, this provides an isomorphism between $Z(\lambda)$ and the ring Sym of symmetric functions.

Given a 2-endomorphism $f: \mathbf{1}_{\lambda^{\prime}} x \mathbf{1}_{\lambda} \Rightarrow \mathbf{1}_{\lambda^{\prime}} x \mathbf{1}_{\lambda}$ in $\mathcal{U}^{*}\left(\mathfrak{s l}_{n}\right)$, we can interpret the class $[f]$ as a diagram on the annulus. If we forget that the diagram is on an annulus, we are left with a diagram representing 
an endomorphism of $\mathbf{1}_{\lambda^{\prime}}$.

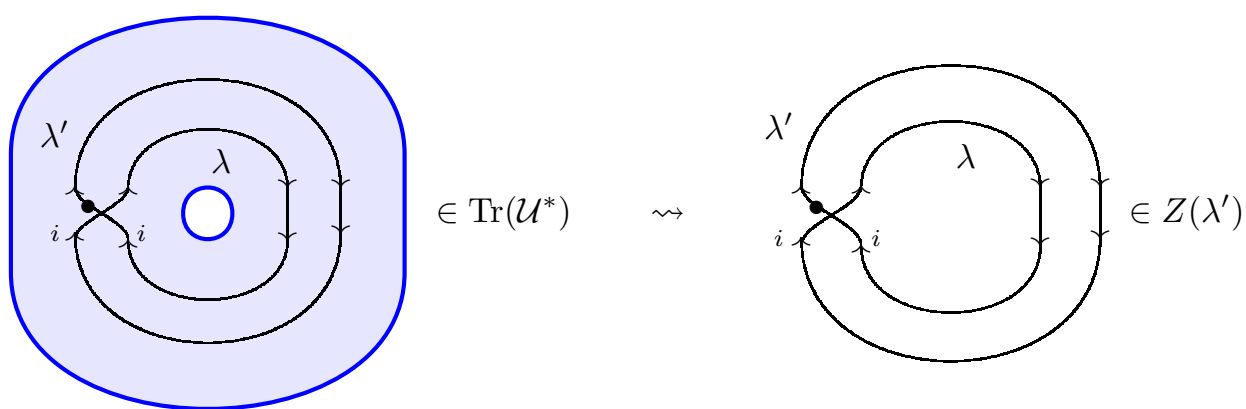

By choosing scalars $t_{i} j=t_{j i}=1$ for all $i \neq j$, the 2-category $\left.\left.\mathcal{U}\left(\mathfrak{s l}_{n}\right)\right)=\mathcal{U}_{Q}\left(\mathfrak{s l}_{n}\right)\right)$ is cyclic and the resulting diagram is independent of the choice of representative for the class $[f] \in \operatorname{Tr}\left(\mathcal{U}\left(\mathfrak{s l}_{n}\right)\right)$. In this way, $[f]$ gives rise to a map $Z(\lambda) \rightarrow Z\left(\lambda^{\prime}\right)$ given by placing an element from $Z(\lambda)$ inside the annulus and regarding the result as an element in $Z\left(\lambda^{\prime}\right)$. Hence, we have the following proposition.

Proposition 6.1. The linear category $\operatorname{Tr}\left(\mathcal{U}^{*}\left(\mathfrak{s l}_{n}\right)\right)$ acts by endomorphisms on the commutative ring $Z\left(\mathcal{U}^{*}\left(\mathfrak{s l}_{n}\right)\right)$. Under this action the class $[f]$ of endomorphisms $f: \mathbf{1}_{\lambda^{\prime}} x \mathbf{1}_{\lambda} \Rightarrow \mathbf{1}_{\lambda^{\prime}} x \mathbf{1}_{\lambda}$ in $\mathcal{U}^{*}\left(\mathfrak{s l}{ }_{n}\right)$ is sent to the linear operator sending a closed diagram $D$ representing an element of $Z(\lambda)$ to the element in $Z\left(\lambda^{\prime}\right)$ obtained by removing the annulus from the diagram $[f . D]$.

Remark for experts. Being able to define an action of the trace on the center of objects in a 2category essentially amounts to having enough "coherent" duality in the 2-category so that the trace takes values in the center of objects. This idea is captured by the notion of a pivotal 2-category [52]. This can be seen as a many object version of a pivotal monoidal category, see 24] where traces in this context are studied. Müger points out in [52, page 11] a strict pivotal 2-category can be defined from Mackaay's work [4] on spherical 2-categories by ignoring the monoidal structure. It is also equivalent to a 2-category in which every 1-morphism has a specified cyclic biadjoint (see [20, 37]).

6.0.2. A current algebra action on centers. In what follows we represent a generic 2-morphism $f \in Z(\lambda)$, $f: \mathbf{1}_{\lambda} \Rightarrow \mathbf{1}_{\lambda}$, by the diagram

$\lambda$

where we use the $*$ to represent any linear combination of closed diagrams describing $f$.

Theorem 6.2. The vector space

$$
Z:=\bigoplus_{\lambda \in \dot{\mathcal{U}}} Z(\lambda)
$$

is a $\mathbf{U}\left(\mathfrak{s l}_{n}[t]\right)$-module with

$$
\begin{array}{rlll}
1_{\lambda}: Z & \longrightarrow & Z(\lambda) \\
\lambda^{\prime} & & \\
* & & \mapsto & \delta_{\lambda, \lambda^{\prime}}\left(\begin{array}{c} 
\\
*
\end{array}\right. \\
& & & \lambda \\
x_{i, r}^{+}: Z(\lambda) & \longrightarrow & Z\left(\lambda+\alpha_{i}\right)
\end{array}
$$




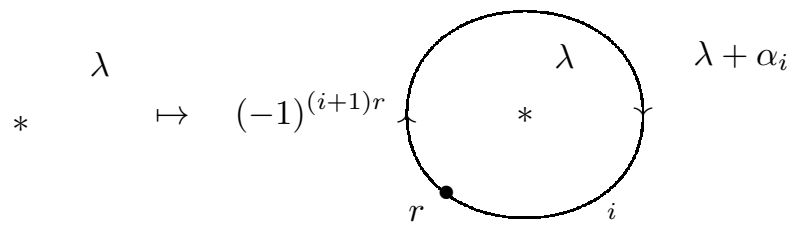

$$
x_{i, s}^{-}: Z(\lambda) \longrightarrow Z\left(\lambda-\alpha_{i}\right)
$$

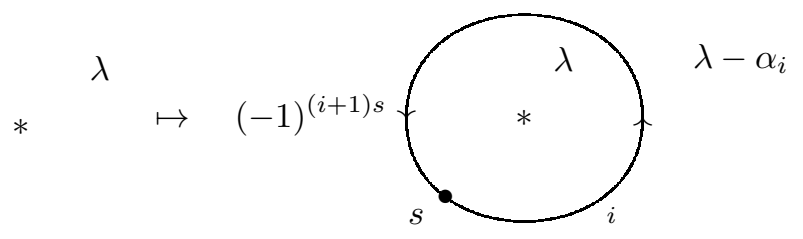

Proof. This theorem follows immediately from Proposition 23. In particular, the fact that the current algebra relations hold in the trace $\operatorname{Tr}\left(\mathcal{U}^{*}\left(\mathfrak{s l}_{n}\right)\right)$ imply that these relations hold under the action described above.

6.0.3. An action of the current algebra on centers of 2-representations.

Definition 6.3. A 2-representation of $\dot{\mathcal{U}}_{Q}\left(\mathfrak{s l}_{n}\right)$ is a graded additive $\mathbb{k}$-linear 2-functor $\Psi: \dot{\mathcal{U}}_{Q}\left(\mathfrak{s l} l_{n}\right) \rightarrow \mathcal{K}$ for some graded, additive 2 -category $\mathcal{K}$.

When all of the Hom categories $\mathcal{K}(x, y)$ between objects $x$ and $y$ of $\mathcal{K}$ are idempotent complete, in other words $\operatorname{Kar}(\mathcal{K}) \cong \mathcal{K}$, then any graded additive $\mathbb{k}$-linear 2 -functor $\mathcal{U}_{Q}\left(\mathfrak{s l}_{n}\right) \rightarrow \mathcal{K}$ extends uniquely to a 2-representation of $\dot{\mathcal{U}}_{Q}\left(\mathfrak{s l}_{n}\right)$.

Any 2-representation $\Psi$ induces a map on traces

$$
\begin{aligned}
\operatorname{Tr}\left(\dot{\mathcal{U}}_{Q}\left(\mathfrak{s l}_{n}\right)\right) & \rightarrow \operatorname{Tr}(\mathcal{K}) \\
{[f] } & \mapsto[\Psi(f)] .
\end{aligned}
$$

When looking at $\mathcal{U}^{*}$ as

$$
\mathcal{U}^{*}(x, y):=\bigoplus_{t \in \mathbb{Z}} \mathcal{U}(x, y\langle t\rangle)
$$

we can easily extend this map to

$$
\operatorname{Tr}\left(\mathcal{U}^{*}\left(\mathfrak{s l}_{n}\right)\right) \rightarrow \operatorname{Tr}(\mathcal{K}) .
$$

Hence by the same procedure described in Proposition 6.1 the trace $\operatorname{Tr}\left(\mathcal{U}^{*}\left(\mathfrak{s l}_{n}\right)\right)$ acts on the center $Z(\mathcal{K})$ of any 2-representation. Since Proposition 23 shows that the current algebra is contained in the trace, it follows that the current algebra acts on any 2-representation.

\section{REFERENCES}

[1] S. Al-Nofayee. Simple objects in the heart of a t-structure. J. Pure Appl. Algebra, 213(1):54-59, 2009.

[2] J. Baez and J. Dolan. Categorification. In Higher category theory (Evanston, IL, 1997), volume 230 of Contemp. Math., pages 1-36. Amer. Math. Soc., Providence, RI, 1998. arXiv:9802029 
[3] M. Balagovic. Degeneration of trigonometric dynamical difference equations for quantum loop algebras to trigonometric Casimir equations for Yangians. 2013. arXiv:1308.2347.

[4] D. Bar-Natan. Khovanov's homology for tangles and cobordisms. Geom. Topol., 9:1443-1499, 2005. arXiv:math/0410495

[5] D. Bar-Natan and S. Morrison. The Karoubi envelope and Lee's degeneration of Khovanov homology. Algebr. Geom. Topol., 6:1459-1469, 2006. arXiv:math/0606542

[6] A. Beilinson, J. Bernstein, and P. Deligne. Faisceaux pervers. In Analysis and topology on singular spaces, I (Luminy, 1981), volume 100 of Astérisque, pages 5-171. Soc. Math. France.

[7] A. Beliakova, K. Habiro, A. Lauda, and B. Webster. Current algebras and categorified quantum groups. in preparation.

[8] A. Beliakova, K. Habiro, A. Lauda, and M. Zivkovic. Trace decategorification of categorified quantum sl(2). in preparation, 2014. arXiv:1404.1806.

[9] C. Blanchet. An oriented model for Khovanov homology. J. Knot Theory Ramifications, 19(2):291-312, 2010.

[10] F. Borceux. Handbook of categorical algebra. 1, volume 50 of Encyclopedia of Mathematics and its Applications. Cambridge University Press, Cambridge, 1994.

[11] J. Brichard. The center of the nilcoxeter and 0-Hecke algebras. 2008. arXiv:0811.2590

[12] J. Brundan. Symmetric functions, parabolic category $\mathcal{O}$, and the Springer fiber. Duke Math. J., 143(1):41-79, 2008. arXiv:math/0608235

[13] J. Brundan and A. Kleshchev. Blocks of cyclotomic Hecke algebras and Khovanov-Lauda algebras. Invent. Math., 178(3):451-484, 2009. arXiv:0808.2032

[14] J. Brundan and A. Kleshchev. Graded decomposition numbers for cyclotomic Hecke algebras. Advances in Math., (222):1883-1942, 2009. arXiv:0901.4450.

[15] J. Brundan and V. Ostrik. Cohomology of Spaltenstein varieties. Transform. Groups, 16(3):619-648, 2011. arXiv:1012.3426

[16] A. Căldăraru and S. Willerton. The Mukai pairing. I. A categorical approach. New York J. Math., 16:61-98, 2010. arXiv:0707.2052

[17] S. Cautis. Clasp technology to knot homology via the affine Grassmannian. 2012. arXiv:1207.2074

[18] S. Cautis, J. Kamnitzer, and S. Morrison. Webs and quantum skew howe duality. 2012. arXiv:1210.6437

[19] S. Cautis and A. D. Lauda. Implicit structure in 2-representations of quantum groups. Selecta Mathematica, 2014. arXiv:1111.1431

[20] J. R. B. Cockett, J. Koslowski, and R. A. G. Seely. Introduction to linear bicategories. Math. Structures Comput. Sci., 10(2):165-203, 2000. The Lambek Festschrift: mathematical structures in computer science (Montreal, QC, 1997).

[21] B. Cooper and V. Krushkal. Handle slides and localizations of categories. Int. Math. Res. Not. IMRN, (10):21792202, 2013. arXiv:1110.2082.

[22] N. Ganter and M. Kapranov. Representation and character theory in 2-categories. Adv. Math., 217(5):2268-2300, 2008. arXiv:math/0602510.

[23] H. Garland. The arithmetic theory of loop algebras. J. Algebra, 53(2):480-551, 1978.

[24] N. Geer, B. Patureau-Mirand, and A. Virelizier. Traces on ideals in pivotal categories. Quantum Topol., 4(1):91-124, 2013.

[25] V. Ginzburg. Lagrangian construction of the enveloping algebra $U\left(\mathrm{sl}_{n}\right)$. C. R. Acad. Sci. Paris Sér. I Math., 312(12):907-912, 1991.

[26] A. Joyal and R. Street. The geometry of tensor calculus. I. Adv. Math., 88(1):55-112, 1991.

[27] M. Kashiwara. On crystal bases of the $Q$-analogue of universal enveloping algebras. Duke Math. J., 63(2):465-516, 1991.

[28] M. Kashiwara. Global crystal bases of quantum groups. Duke Math. J., 69(2):455-485, 1993.

[29] L.H. Kauffman. Spin networks and knot polynomials. Internat. J. Modern Phys. A, 5(1):93-115, 1990.

[30] D. Kazhdan and G. Lusztig. A topological approach to Springer's representations. Adv. in Math., 38(2):222-228, 1980.

[31] M. Khovanov. A categorification of the Jones polynomial. Duke Math. J., 101(3):359-426, 2000. arXiv:9908171

[32] M. Khovanov. A functor-valued invariant of tangles. Algebr. Geom. Topol., 2:665-741 (electronic), 2002. arXiv:0103190

[33] M. Khovanov and A. Lauda. A diagrammatic approach to categorification of quantum groups III. Quantum Topology, 1:1-92, 2010. arXiv:0807.3250

[34] M. Khovanov, A. Lauda, M. Mackaay, and M. Stošić. Extended graphical calculus for categorified quantum sl(2). Memoirs of the AMS, 219, 2012. arXiv:1006.2866.

[35] M. Khovanov, V. Mazorchuk, and C. Stroppel. A brief review of abelian categorifications. Theory Appl. Categ., 22:No. 19, 479-508, 2009. arXiv:0702746 
[36] G. Kuperberg. Spiders for rank 2 Lie algebras. Comm. Math. Phys., 180(1):109-151, 1996. arXiv:9712003

[37] A. D. Lauda. A categorification of quantum sl(2). Adv. Math., 225:3327-3424, 2008. arXiv:0803.3652

[38] A. D. Lauda. An introduction to diagrammatic algebra and categorified quantum $\mathfrak{s l}_{2}$. Bulletin Inst. Math. Academia Sinica, 7:165-270, 2012. arXiv:1106.2128

[39] A. D. Lauda and M. Vazirani. Crystals from categorified quantum groups. Adv. Math., 228(2):803-861, 2011. arXiv:0909.1810

[40] A.D. Lauda, H. Queffelec, and D. Rose. Khovanov homology is a skew howe 2-representation of categorified quantum $\mathrm{sl}(\mathrm{m})$. arXiv: 1212.6076

[41] J.L. Loday. Cyclic homology, volume 301 of Fundamental Principles of Mathematical Sciences. Springer-Verlag, Berlin, second edition, 1998.

[42] G. Lusztig. Canonical bases arising from quantized enveloping algebras. J. Amer. Math. Soc., 3(2):447-498, 1990.

[43] G. Lusztig. Canonical bases arising from quantized enveloping algebras. II. Progr. Theoret. Phys. Suppl., (102):175201 (1991), 1990. Common trends in mathematics and quantum field theories (Kyoto, 1990).

[44] G. Lusztig. Canonical bases in tensor products. Proc. Nat. Acad. Sci. U.S.A., 89(17):8177-8179, 1992.

[45] G. Lusztig. Introduction to quantum groups, volume 110 of Progress in Mathematics. Birkhäuser Boston Inc., Boston, MA, 1993.

[46] I. G. Macdonald. Symmetric functions and Hall polynomials. The Clarendon Press Oxford University Press, New York, 1979. Oxford Mathematical Monographs.

[47] M. Mackaay. Spherical 2-categories and 4-manifold invariants. Adv. Math., 143(2):288-348, 1999. arXiv:math/9805030

[48] M. Mackaay. sl(3)-foams and the Khovanov-Lauda categorification of quantum sl(k). 2009. arXiv:0905.2059

[49] J. Milnor. Introduction to algebraic K-theory. Princeton University Press, Princeton, N.J., 1971. Annals of Mathematics Studies, No. 72.

[50] B. Mitchell. Rings with several objects. Advances in Math., 8:1-161, 1972.

[51] S. Morrison. A diagrammatic category for the representation theory of (Uqsln ). ProQuest LLC, Ann Arbor, MI, 2007. Thesis (Ph.D.)-University of California, Berkeley.

[52] M. Müger. From subfactors to categories and topology. I. Frobenius algebras in and Morita equivalence of tensor categories. J. Pure Appl. Algebra, 180(1-2):81-157, 2003. arXiv:0111204

[53] R. Penrose. Applications of negative dimensional tensors. In Combinatorial Mathematics and its Applications (Proc. Conf., Oxford, 1969), pages 221-244. Academic Press, London, 1971.

[54] H. Queffelec and D.E.V. Rose. The $s l(n)$ foam category: a combinatorial formulation of Khovanov-Rozansky homology via categorical skew howe duality. 2014. arXiv:1405.5920

[55] N. Reshetikhin and V. Turaev. Ribbon graphs and their invariants derived from quantum groups. Comm. Math. Phys., 127(1):1-26, 1990.

[56] J. Rosenberg. Algebraic K-theory and its applications, volume 147 of Graduate Texts in Mathematics. SpringerVerlag, New York, 1994.

[57] R. Rouquier. 2-Kac-Moody algebras, 2008. arXiv:0812.5023.

[58] A. Savage. Introduction to categorification. 2014. arXiv:1401.6037

[59] R. Street. Low-dimensional topology and higher-order categories. In Proceedings of CT95, Halifax, July 9-15, 1995.

[60] V.G. Turaev. Quantum invariants of knots and 3-manifolds, volume 18 of De Gruyter Studies in Math.

[61] M. Varagnolo and E. Vasserot. Canonical bases and KLR-algebras. J. Reine Angew. Math., 659:67-100, 2011.

[62] B. Webster. Knot invariants and higher representation theory I: diagrammatic and geometric categorification of tensor products. 2010. arXiv:1001.2020

[63] B. Webster. Canonical bases and higher representation theory. 2012. arXiv:1209.0051

[64] B. Webster. Knot invariants and higher representation theory. 2013. arXiv:1309.3796.

[65] M. Zivkovic. Trace decategorification of the quantum $\mathfrak{s l}_{3}, 2014$. arXiv:1405.2314.

Universität ZÜRICH, WinterthURerstr. 190 CH-8057 ZÜrich, SwitZerland

E-mail address: anna@math.uzh.ch

Universität Zürich, Winterthurerstr. 190 CH-8057 ZÜrich, SwitZerland

E-mail address: zaur.guliyev@math.uzh.ch

Research Institute for Mathematical Sciences, Kyoto University, Kyoto, 606-8502, Japan

E-mail address: habiro@kurims.kyoto-u.ac.jp

University of Southern California, Los Angeles, CA 90089, USA

E-mail address: lauda@usc.edu 